\title{
Giving an Adversary Guarantees (Or: How to Model Designated Verifier Signatures in a Composable Framework)
}

\section{Conference Paper}

Author(s):

Maurer, Ueli; Portmann, Christopher (1); Rito, Guilherme

Publication date:

2021-12-05

Permanent link:

https://doi.org/10.3929/ethz-b-000520644

Rights / license:

$\underline{\text { In Copyright - Non-Commercial Use Permitted }}$

Originally published in:

Lecture Notes in Computer Science 13092, https://doi.org/10.1007/978-3-030-92078-4_7 


\title{
Giving an Adversary Guarantees (Or: How to Model Designated Verifier Signatures in a Composable Framework)
}

\author{
Ueli Maurer $^{1}$, Christopher Portmann ${ }^{2 \star}$, and Guilherme Rito ${ }^{1}$ (D) \\ 1 Department of Computer Science, ETH Zürich, Switzerland \\ \{maurer,gteixeir\}@inf .ethz.ch \\ 2 Concordium, Zürich, Switzerland \\ cp@concordium.com
}

\begin{abstract}
When defining a security notion, one typically specifies what dishonest parties cannot achieve. For example, communication is confidential if a third party cannot learn anything about the messages being transmitted, and it is authentic if a third party cannot impersonate the real (honest) sender. For certain applications, however, security crucially relies on giving dishonest parties certain capabilities. As an example, in Designated Verifier Signature (DVS) schemes, one captures that only the designated verifier can be convinced of the authenticity of a message by guaranteeing that any dishonest party can forge signatures which look indistinguishable (to a third party) from original ones created by the sender.

However, composable frameworks cannot typically model such guarantees as they are only designed to bound what a dishonest party can do. In this paper we show how to model such guarantees - that dishonest parties must have some capability - in the Constructive Cryptography framework (Maurer and Renner, ICS 2011). More concretely, we give the first composable security definitions for Multi-Designated Verifier Signature (MDVS) schemes - a generalization of DVS schemes.

The ideal world is defined as the intersection of two worlds. The first captures authenticity in the usual way. The second provides the guarantee that a dishonest party can forge signatures. By taking the intersection we have an ideal world with the desired properties.

We also compare our composable definitions to existing security notions for MDVS schemes from the literature. We find that only recently, 23 years after the introduction of MDVS schemes, sufficiently strong security notions were introduced capturing the security of MDVS schemes (Damgård et al., TCC 2020). As we prove, however, these notions are still strictly stronger than necessary.
\end{abstract}

* Work done while author was at ETH Zürich, Switzerland. 


\section{Introduction}

\subsection{Composable security}

In a nutshell, composable security frameworks define security by designing an ideal world and proving that the real world is indistinguishable $2,5,8,12,20,22$ 23. 26. Typically, one first designs an ideal functionality, which corresponds to the functionality one wishes to achieve. For example, if one wants confidential communication from Alice to Bob, then the ideal functionality allows Alice to input messages, Bob to read messages, and guarantees that Eve can only learn the length of the messages input by Alice. Eve could additionally be given extra capabilities that do not violate confidentiality, e.g. inputting messages. A simulator is then connected to this ideal functionality, covering the idealized inputs and outputs available to dishonest parties and providing "real" inputs and outputs to the environment (that should be indistinguishable from those of the real world). Let $\mathbf{S}$ denote an ideal functionality, and $\operatorname{sim} \mathbf{S}$ the ideal world consisting of $\mathbf{S}$ with some simulator sim attached. Since any (efficient) simulator $\operatorname{sim} \in \Omega$ is acceptable, one can alternatively view the ideal world as the set of all possible acceptable ideal worlds:

$$
\mathcal{S}=\{\operatorname{sim} \mathbf{S}\}_{\operatorname{sim} \in \Omega} .
$$

A security proof then shows that the real world $\mathcal{R}$ (also modeled as a set) is a subset of the ideal world $\mathcal{S}$. Since sim covers the dishonest parties' interfaces of $\mathbf{S}$, it can only further limit the capabilities of dishonest parties. For example, an ideal functionality for confidentiality might allow a third party to change Alice's message, but if this is not possible in the real world, the simulator can disallow the environment to use that capability. This structure of the ideal world makes it impossible for traditional composable frameworks to provide guarantees about a dishonest party's capabilities, because these might be blocked by the simulator.

Some prior works using the Constructive Cryptography (CC) framework 14.23 have noted that the ideal world does not have to be structured as in Eq. (1.1). In particular, the simulator does not have to necessarily cover all dishonest parties' interfaces (or might not be present at all). This relaxed view of the ideal world allows one to define composable security notions capturing the security of schemes whose security could not be modeled by traditional composable frameworks. In this work we crucially exploit this to give the first composable security notions for Multi-Designated Verifier Signature schemes. We refer the interested reader to [3] to see how to model Digital Signature Schemes (DSS) in CC, and to 14] for an extended introduction to CC, in which some of the novel techniques used here were first applied.

\subsection{MDVS schemes}

Designated Verifier Signature (DVS) schemes are a variant of DSSthat allow a signer to sign messages towards a specific receiver, chosen (or designated) by the 
signer $9,11,13,16,19,27,30,32$. The goal of these schemes is to establish an authentic communication channel, say from a sender Alice to a receiver Bob, where the authenticity property is exclusive to the receiver Bob designated by Alice, i.e. Bob and only Bob can tell whether Alice actually sent some message authentically. In Multi-Designated Verifier Signature (MDVS) schemes 9, 11, 13 16,32 , multiple receivers may be designated verifiers for the same message, e.g. Alice signs a message so that both Bob and Charlie can verify that Alice generated the signature, but a third party Eve would not be convinced that Alice signed it. This should hold even if a verifier is dishonest, say Bob, and provides his secret keys to Eve. MDVS schemes achieve this by guaranteeing that Bob could forge signatures that would look indistinguishable to Eve from Alice's signatures - but Charlie could distinguish the two using his secret key, thus authenticity with respect to the designated verifiers is not violated.

MDVS schemes have numerous applications: from secure messaging (and in particular secure group messaging for the multi-verifier case) [11, to online auctions wherein all bidders place their binding-bids in a non-interactive way, and the highest bidder wins. In the case of online auctions a bidder Bob would then sign its bid to both the auctioneer Charlie and his bank Blockobank, and if Bob wins Charlie would then sign a document stating Bob is the winner of the auction; the winner could also be kept anonymous by having Charlie signing such document only with respect to Bob, its bank Blockobank and any other official entity needed to confirm Bob's ownership of the auctioned item.

While composable security notions for DSS are well understood [1, 3, 5, 6, the literature on (M)DVS schemes provides only a series of different game-based security definitions - which we discuss in detail in Sect. 6 capturing a variety of properties that an MDVS scheme could possess. By defining the ideal world for an MDVS scheme in this work, we can compare the resulting composable definition to the game-based ones and determine which security properties are needed. It turns out that crucial properties for the security of MDVS schemes like consistency - all (honest) designated verifiers will either accept or reject the same signature - and security against any subset of dishonest verifiers were only introduced very recently [11].

\subsection{Contributions}

Providing guarantees to Dishonest Parties. To capture that a dishonest party is guaranteed to have some capability, we introduce a new type of ideal world specification, which we sketch in this section. The first step consists in defining a set of ideal functionalities (called resources or resource specification in CC 22,23$]$ ) that have the required property. For example, in the case of MDVS schemes, we want a dishonest receiver to be able to generate a valid signature. This corresponds to a channel in which both Alice (the honest sender) and Bob (the dishonest receiver) may insert messages. Thus anyone reading from that channel

would not know if the message is from Alice or Bob. Let $\widehat{\mathcal{X}}$ denote such a set. The ideal worlds we are interested in are those in which a dishonest receiver 
could achieve this property if they run an (explicit) forging algorithm $\pi$. Thus, the ideal world of interest is defined as

$$
\mathcal{X}=\{\mathbf{X}: \pi \mathbf{X} \in \widehat{\mathcal{X}}\}
$$

where $\pi \mathbf{X}$ denotes a resource $\mathbf{X}$ with the algorithm $\pi$ being run at the dishonest receivers' interface of $\mathbf{X}$.

Similar techniques could be used to model ideal worlds for ring signatures 427 or coercibility [22,31].

Composable Security Notions for MDVS Schemes. We then use the technique described above to define composable security for MDVS schemes. For example, if one considers a fixed honest sender and a fixed set of designated verifiers (some of which may be dishonest), then an MDVS scheme is expected to achieve authenticity with respect to the honest verifiers, but this authenticity should be exclusive to them, meaning that any dishonest player should be able to generate a signature that would fool a third party Eve. Authenticity is captured in the usual way (see, e.g. [3]), as in Eq. (1.1), i.e. we define an authentic channel $\mathbf{A}$ from Alice to the honest verifiers, and the ideal world is given by a set

$$
\mathcal{A}=\{\operatorname{sim} \mathbf{A}\}_{\text {sim } \in \Omega} .
$$

The exclusiveness of the authenticity is defined with a (set of) ideal world(s) as in Eq. (1.2), Both properties are then achieved by taking the intersection of the two, namely by proving that for the real world $\mathcal{R}$ we have

$$
\mathcal{R} \subseteq \mathcal{A} \cap \mathcal{X}
$$

Comparison With Existing Notions for MDVS. Now that the composable security notion is defined, we compare it to the game-based definitions from the literature. It turns out that only the most recent definitions from [11] are sufficient to achieve composable security.

More precisely, we prove reductions and a separation between our composable security definition and the games of 11]. Our statements imply the following:

- any MDVS scheme which is Correct, Consistent, Unforgeable and Off-TheRecord (according to [11) can be used to construct the ideal world for MDVS;

- there is an MDVS scheme which satisfies the composable definition, but which is not Off-The-Record (as defined in 11]).

\subsection{Structure of this paper}

In Sect. 2 we start by introducing the concepts from CC $14,20,22,23$ that are needed to understand the framework. We also define repositories which are the resources we use in this work for communication between parties jointly running a protocol (see also [3]). In Sect. 3 we introduce MDVS schemes and state the 
game-based security notions from [11 capturing their security. In Sect. 4 we consider a setting in which the sender and designated receivers are fixed and publicly known. This allows us to define the ideal worlds and the corresponding composable security definition in a simpler setting. Also for simplicity, we only require that dishonest delegated verifiers have the ability to forge signatures, not third parties. We then prove that the security games from 11 are sufficient to imply composable security. In Sect. 5 we model the more general setting where the sender and designated receivers can be arbitrarily chosen. As before, we model composable security and prove that the security games from [11 are sufficient to achieve composable security in this setting as well. But we also prove a seperation between the Off-The-Record game from [11 and the composable security defintion, showing that this game is stronger than necessary. Note that in this section any dishonest party should be able to forge signatures, not only the dishonest designated verifiers. Finally, in Sect. 6 we discuss the literature related to MDVS schemes and some of the issues in previous security definitions.

\section{Constructive Cryptography}

The Constructive Cryptography (CC) framework 20.22 views cryptography as a resource theory: protocols construct new resources from existing (assumed) ones. For example, a CCA-secure encryption scheme constructs a confidential channel given a public key infrastructure and an insecure channel on which the ciphertext is sent [10]. The notion of resource construction is inherently composable: if a protocol $\pi_{1}$ constructs $\mathcal{R}$ from $\mathcal{S}$ and $\pi_{2}$ constructs $\mathcal{T}$ from $\mathcal{S}$, then running both protocols will construct $\mathcal{T}$ given that one initially has access to $\mathcal{R} 3^{3}$

In this section we first review the building blocks of $\mathrm{CC}$ in Sect. 2.1. We explain how security is defined in Sect. 2.2. Then in Sect. 2.3 we model a specific type of resources, namely repositories, which is an abstract model of communication. Throughout the rest of the paper, for any set of parties $\mathcal{S}$, we denote by $\mathcal{S}^{H}$ the partition of $\mathcal{S}$ containing all honest parties, and $\overline{\mathcal{S}^{H}}$ the partition containing all dishonest parties, such that $\mathcal{S}=\mathcal{S}^{H} \uplus \overline{\mathcal{S}^{H}}$. The set of all parties is denoted $\mathcal{P}$.

\subsection{Resource Specifications, Converters, and Distinguishers}

Resource. A resource is an interactive system shared by all parties, e.g. a channel or a key resource - and is akin to an ideal functionality in UC [5]. Each party can provide inputs and receive outputs from the resource. We use the term interface to denote specific subsets of the inputs and outputs, in particular, all the inputs and outputs available to a specific party are assigned to that party's interface. For example, an insecure channel INS allows all parties to input messages at their interface and read the contents of the channel. A confidential channel resource CONF shared between a sender Alice, a receiver Bob and an eavesdropper Eve allows Alice to input messages at her interface; it allows Eve to insert her own messages and it allows her to duplicate Alice's messages, but not to read them

\footnotetext{
${ }^{3}$ For a formal statement of the composition theorem used here we refer to $14,23$.
}

${ }^{4}$ More precisely, the CONF channel only allows Eve to read the length of messages. 
and it allows Bob to receive at his interface any of the messages inserted by Alice or Eve. As another example, an authenticated channel from Bob to Alice (AUT) allows Bob to send messages through the channel and allows Alice and Eve to read messages from the channel.

Formally, a resource is a random system 24,25 , i.e. it is uniquely defined by a sequence of conditional probability distributions. For simplicity, however, we usually describe resources by pseudo-code.

If multiple resources $\left\{\mathbf{R}_{i}\right\}_{i=1}^{n}$ are simultaneously accessible, we write $\mathbf{R}=$ $\left[\mathbf{R}_{1}, \ldots, \mathbf{R}_{n}\right]$, or alternatively $\mathbf{R}=\left[\mathbf{R}_{i}\right]_{i \in\{1, \ldots, n\}}$, for the new resource obtained by the parallel composition of all $\mathbf{R}_{i}$, i.e. $\mathbf{R}$ is a resource that provides each party with access to the (sub)resources $\mathbf{R}_{i}$.

Converter. A converter is an interactive system executed either locally by a single party or cooperatively by multiple parties. Its inputs and outputs are partitioned into an inside interface and an outside interface. The inside interface connects to (those parties' interfaces of) the available resources, resulting in a new resource. For instance, connecting a converter $\alpha$ to Alice's interface $A$ of a resource $\mathbf{R}$ results in a new resource, which we denote by $\alpha^{A} \mathbf{R}$. The outside interface of the converter $\alpha$ is now the new $A$-interface of $\alpha^{A} \mathbf{R}$. Thus, a converter may be seen as a map between resources. Note that converters applied at different interfaces commute, i.e. $\beta^{B} \alpha^{A} \mathbf{R}=\alpha^{A} \beta^{B} \mathbf{R}$.

A protocol is given by a tuple of converters $\pi=\left(\pi_{P_{i}}\right)_{P_{i} \in \mathcal{P}^{H}}$, one for each (honest) party $P_{i} \in \mathcal{P}^{H}$. Simulators are also given by converters. For any set $\mathcal{S}$ will often write $\pi^{\mathcal{S}} \mathbf{R}$ for $\left(\pi_{P_{i}}\right)_{P_{i} \in \mathcal{S}} \mathbf{R}$. We also often drop the interface superscript and write just $\pi \mathbf{R}$ when it is clear from the context to which interfaces $\pi$ connects.

For example, suppose Alice and Bob share an insecure channel INS and a single use authenticated channel from Bob to Alice AUT and suppose that Alice runs a converter enc and Bob runs a converter dec, and that these converters behave as follows: First, converter dec generates a public-secret key-pair (pk,sk) for Bob and sends pk over the single-use authenticated channel AUT to Alice. Each time a message $m$ is input at the outside interface of enc, the converter uses Bob's public key pk - which it received from AUT - to compute a ciphertext $c=E n c_{\mathrm{pk}}(m)$; it then sends this ciphertext over the insecure channel to Bob (via the inside interface of enc connected to INS). Each time Bob's decryption converter dec receives a ciphertext $c$ from the INS channel, it uses Bob's secret key sk to decrypt $c$, obtaining a message $m=D e c_{\mathrm{sk}}(c)$, and if $m$ is a valid plaintext, the converter then outputs $m$ to Bob (via the outside interface of the converter). The real world of such a system is given by

$$
\operatorname{dec}^{B} \text { enc }^{A}[\mathbf{A U T}, \mathbf{I N S}] .
$$

Specification. Often one is not interested in a unique resource, but in a set of resources with common properties. For example, the confidential channel described above allows Eve to insert messages of her own. Yet, if she did not have this ability, the resulting channel would still be a confidential one. We call such a 
set a resource specification (or simply also a resource), and denote it with a bold calligraphic letter, e.g. a specification of confidential channels could be defined as

$$
\mathcal{T}=\left\{\operatorname{sim}^{E} \mathbf{C O N F}\right\}_{\operatorname{sim} \in \Omega}
$$

where $\Omega$ is a set of converters (the simulators) that are applied at Eve's interface ${ }^{5}$

Parallel composition of specifications $\mathcal{R}$ and $\mathcal{S}$, and composition of a converter $\alpha$ and a specification $\mathcal{R}$ follow by applying the operations elementwise to the resources $\mathbf{R} \in \mathcal{R}$ and $\mathbf{S} \in \mathcal{S}$.

Distinguisher. To measure the distance between two resources we use the standard notion of a distinguisher, an interactive system $\mathbf{D}$ which interacts with a resource at all its interfaces, and outputs a bit 0 or 1 . The distinguishing advantage for distinguisher $\mathbf{D}$ is defined as

$$
\Delta^{\mathbf{D}}(\mathbf{R}, \mathbf{S}):=\operatorname{Pr}[\mathbf{D S}=1]-\operatorname{Pr}[\mathbf{D R}=1]
$$

where $\mathbf{D R}$ and $\mathbf{D S}$ are the random variables over the output of $\mathbf{D}$ when it interacts with $\mathbf{R}$ and $\mathbf{S}$, respectively.

Relaxation. Typically one proves that the ability to distinguish between two resources is bounded by some function of the distinguisher, e.g. for any $\mathbf{D}$,

$$
\left|\Delta^{\mathbf{D}}(\mathbf{R}, \mathbf{S})\right| \leq \varepsilon(\mathbf{D})
$$

where $\varepsilon(\mathbf{D})$ might be the probability that $\mathbf{D}$ can win a game or solve some finite instance of a problem believed to be hard ${ }^{6}$

This distance measure then naturally defines another type of specification, namely an $\varepsilon$-ball: for a resource specification $\mathcal{R}$, the $\varepsilon$-ball around $\mathcal{R}$ is given by

$$
\mathcal{R}^{\varepsilon}:=\bigcup_{\mathbf{R} \in \mathcal{R}}\left\{\mathbf{S}: \forall \mathbf{D},\left|\Delta^{\mathbf{D}}(\mathbf{R}, \mathbf{S})\right| \leq \varepsilon(\mathbf{D})\right\}
$$

If one chooses a function $\varepsilon(\mathbf{D})$ which is small for a certain class of distinguishers $\mathbf{D}$ - e.g. $\varepsilon(\mathbf{D})$ is small for all $\mathbf{D}$ that cannot be used to solve (a finite instance of) a problem believed to be hard, as described in Footnote 6 -but potentially large for other $\mathbf{D}$, then we have a specification of resources that are indistinguishable (to the distinguishers in the chosen class) from (one of) those in $\mathcal{R}$.

\footnotetext{
${ }^{5}$ The definition of the set $\Omega$ may depend on the context, e.g. whether one is interested in bounded run time, bounded memory, and whether one is making finite or asymptotic statements.

${ }^{6}$ Formally, one first finds an (efficient) reduction $\chi$ which constructs a solver $\mathbf{S}=$ $\chi(\mathbf{D})$ from any distinguisher $\mathbf{D}$. Then one bounds the distance $\left|\Delta^{\mathbf{D}}(\mathbf{R}, \mathbf{S})\right|$ with a function of the probability that $\chi(\mathbf{D})$ succeeds is solving some problem, i.e., $\varepsilon(\mathbf{D}):=f(\operatorname{Pr}[\chi(\mathbf{D})$ succeeds $])$ for an $f$ that does not significantly alter the probability of success. Thus for any $\mathbf{D}$ that cannot be used to solve the problem, $\left|\Delta^{\mathbf{D}}(\mathbf{R}, \mathbf{S})\right|$ must be small.
} 
Remark 1 (Finite vs. Asymptotic security statements). In this paper, rather than making asymptotic security statements (where one considers the limit $k \rightarrow \infty$ for security parameter $k$ ) we make a security statement for each possible $k \in \mathbb{N}$. Specifications, resources, converters and distinguishers are then defined for a fixed security parameter $k$. If needed, one can obtain the corresponding asymptotic statements by defining sequences of resources, converters and distinguishers and then making a statement about the limit behavior of these sequences when $k \rightarrow \infty$.

\subsection{Composable Security}

We now have all the elements needed to define a cryptographic construction.

Definition 1 (Cryptographic Construction [14,23]). Let $\mathcal{R}$ and $\mathcal{S}$ be two resource specifications, and $\pi$ be a protocol for $\mathcal{R}$. We say that $\pi$ constructs $\mathcal{S}$ from $\mathcal{R}$ if

$$
\pi \mathcal{R} \subseteq \mathcal{S} .
$$

For example, in the case of constructing the confidential channel described above, the real world is the singleton set with the element given in Eq. (2.1), and the ideal world is given by an $\varepsilon$-ball around the set of confidential channels given in Eq. (2.2) i.e. to prove security one would need to show that

$$
\operatorname{dec}^{B} \operatorname{enc}^{A}\{[\mathbf{A U T}, \mathbf{I N S}]\} \subseteq\left(\left\{\operatorname{sim}^{E} \mathbf{C O N F}\right\}_{\operatorname{sim} \in \Omega}\right)^{\varepsilon} .
$$

Equation (2.5) is equivalent to the more traditional notation of requiring the existence of a simulator sim such that for all $\mathbf{D}$,

$$
\mid \Delta^{\mathbf{D}}\left(\operatorname{dec}^{B} \text { enc }^{A}[\mathbf{A U T}, \mathbf{I N S}], \operatorname{sim}^{E} \mathbf{C O N F}\right) \mid \leq \varepsilon(\mathbf{D}) .
$$

But the formulation in Definition 1 is more general and allows other types of ideal worlds to be defined than the specification obtained by appending a simulator at Eve's interface of the ideal resource and taking an $\varepsilon$-ball.

Remark 2 (Asymptotic Construction). As pointed out in Remark 1 specifications, resources, converters and distinguishers are defined for a fixed security parameter $k$. The specifications and converters in Definition 1 are then to be interpreted as being defined for a concrete security parameter $k$, and Eq. (2.4) is to be understood as a statement about a fixed $k$, i.e.

$$
\pi_{k} \mathcal{R}_{k} \subseteq \mathcal{S}_{k}
$$

For simplicity we omit the security parameter whenever it is clear from the context, and thus will simply write as in Eq. (2.4). If one wishes to make an asymptotic security statement then one defines efficient families $\left\{\pi_{k}\right\}_{k \in \mathbb{N}},\left\{\boldsymbol{\mathcal { R }}_{k}\right\}_{k \in \mathbb{N}},\left\{\boldsymbol{\mathcal { S }}_{k}\right\}_{k \in \mathbb{N}}$ and shows that Eq. (2.6) holds asymptotically in $k$, meaning that there is a family $\vec{\varepsilon}:=\left\{\varepsilon_{k}\right\}_{k \in \mathbb{N}}$ of $\varepsilon$-balls such that $\pi_{k} \boldsymbol{\mathcal { R }}_{k} \subseteq\left(\boldsymbol{\mathcal { S }}_{k}\right)^{\varepsilon_{k}}$, and for any efficient family of distinguishers $\overrightarrow{\mathbf{D}}:=\left\{\mathbf{D}_{k}\right\}_{k \in \mathbb{N}}$, the function $\vec{\varepsilon}(\overrightarrow{\mathbf{D}}): \mathbb{N} \rightarrow \mathbb{R}$ defined as $\vec{\varepsilon}(\overrightarrow{\mathbf{D}})(k):=\varepsilon_{k}\left(\mathbf{D}_{k}\right)$ is negligible. 
Remark 3 (Modeling different sets of (dis)honest parties). When one is interested in making security statements for different sets of (dis)honest parties it is not sufficient to make a single statement as in Definition 1. Instead, one makes a statement for each relevant set of (dis)honest parties. For example, let $\pi$ be the protocol defining a converter $\pi_{i}$ for each party $P_{i} \in \mathcal{P}$. For every relevant subset of honest parties $\mathcal{P}^{H} \subseteq \mathcal{P}$, letting $\boldsymbol{\mathcal { R }}^{\mathcal{P}^{H}}$ and $\mathcal{S}^{\mathcal{P}^{H}}$ denote, respectively, the available resources' specifications - the real world - and the desired resources' specifications - the ideal world - one needs to prove that

$$
\pi^{\mathcal{P}^{H}} \mathcal{R}^{\mathcal{P}^{H}} \subseteq \mathcal{S}^{\mathcal{P}^{H}}
$$

where $\pi^{\mathcal{P}^{H}} \mathcal{R}^{\mathcal{P}^{H}}$ denotes the attachment of each converter $\pi_{i}$-run by honest party $P_{i} \in \mathcal{P}^{H}$ as ascribed by the protocol $\pi$ - to $\mathcal{R}^{\mathcal{P}^{H}}$. In this paper, although we will make statements of this format, i.e. modeling different sets of (dis)honest parties, we will drop the superscript $\mathcal{P}^{H}$ from the notation of the converters and specifications, whenever clear from the context.

\subsection{Access Restricted Repositories}

We formalize communication between different parties as having access to a repository resource. More specifically, a repository consists of a set of registers and a single buffer containing register identifiers; a register is a pair reg $=(\mathrm{id}, m)$, which includes the register's identifier id (uniquely identifying the register among all repositories), and a message $m \in \mathcal{M}$ (where $\mathcal{M}$ is the message space of the repository 7 . Access rights to a repository are divided in three classes: write access allows a party to add messages to a repository, read access allows a party to read all the messages in a repository, and copy access allows a party to make duplicates of messages already existing in the repository (without necessarily being able to read the messages) ${ }^{8}$ Let $\mathcal{P}$ be the set of all parties, and let $\mathcal{W} \subseteq \mathcal{P}, \mathcal{R} \subseteq \mathcal{P}$ and $\mathcal{C} \subseteq \mathcal{P}$ denote the parties with write, read and copy access to a repository rep, respectively. We will write ${ }^{\mathcal{C}}$ rep $\mathcal{W}_{\mathcal{R}}^{\mathcal{W}}$ whenever it is needed to make the access permissions explicit. Though we may drop them and only write rep whenever clear from the context. For example, in the three party setting with sender Alice, receiver Bob and dishonest Eve, i.e. $\mathcal{P}=\{A, B, E\}$, the insecure channel mentioned in Sect. 2.1 - which allows all parties to read and write - is given by $\mathbf{I N S}_{\mathcal{P}}^{\mathcal{P}}{ }^{9}$ an authentic channel from Alice to Bob is given by ${ }^{\{E\}} \mathbf{A U T}_{\{B, E\}}^{\{A\}}$; for fixed-length message spaces, the confidential channel mentioned in Sect. 2.1 is

\footnotetext{
${ }^{7}$ In analogy to Remark 1 we consider that a repository defined for security parameter $k$ has message space $\mathcal{M}_{k}$; for a family of repositories one then considers a corresponding family of message spaces $\overrightarrow{\mathcal{M}}:=\left\{\mathcal{M}_{k}\right\}_{k \in \mathbb{N}}$. Since most statements are made for a fixed parameter $k$, we usually omit $k$ from the notation, writing $\mathcal{M}$ instead.

${ }^{8}$ Copy access is used to capture the capability that dishonest parties have for copying or resending (modifications of) whatever they see; modeling this capability is crucial for some of the security proofs.

9 Since all parties can read and write, copying capabilities are redundant.
} 
given by ${ }^{\{E\}} \mathbf{C O N F}_{\{B\}}^{\{A, E\}}$. The exact semantics of such an (atomic) repository are defined in Algorithm 1.

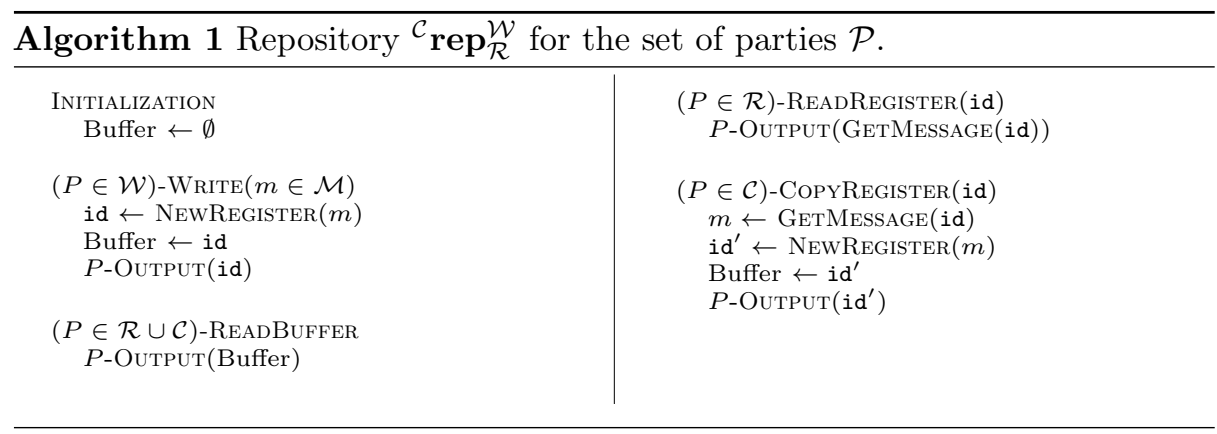

Parties will typically have access to many repositories simultaneously, e.g. an authentic repository from Alice to Bob and one from Alice to Charlie. One could model this as providing all these (atomic) repositories in parallel to the players, i.e.

$$
\left[{ }^{\mathcal{C}_{1}} \operatorname{rep}_{\mathbf{1}}{ }_{\mathcal{R}_{1}}^{\mathcal{W}_{1}}, \ldots,{ }^{\mathcal{C}_{n}} \operatorname{rep}_{\mathbf{n}_{\mathcal{R}_{n}}}^{\mathcal{W}_{n}}\right]
$$

However, this would mean that to check for incoming messages, a party would need to check every possible atomic repository $\mathbf{r e p}_{i}$, which could be inefficient if the number of atomic repositories is very high. Instead, we define a new resource REP which is identical to a parallel composition of the atomic repositories, except that it allows parties to efficiently check for incoming messages (rather than requiring parties to poll each atomic repository $\mathbf{r e p}_{\mathbf{i}}$ they have access to). Abusing notation, we denote such a resource as in Eq. (2.7) namely

$$
\mathbf{R E P}=\left[{ }^{\mathcal{C}_{1}} \operatorname{rep}_{\mathbf{1}_{\mathcal{R}_{1}}}^{\mathcal{W}_{1}}, \ldots,{ }^{\mathcal{C}_{n}} \operatorname{rep}_{\mathbf{n}_{\mathcal{R}_{n}}}{ }^{\mathcal{W}_{n}}\right]
$$

The new resource REP allows every party with read or copy access to issue a single READBUFFER operation that returns a list of pairs, each pair containing a register's identifier and a label identifying the atomic repository in which the register was written. In addition, it provides single READREGISTER and COPYREgister operations which return the contents of the register with the given id and copy the register with the given id, respectively. WRITE operations for REP additionally have to specify the atomic repository for which the operation is meant. The exact semantics of REP are defined in Algorithm 2

\section{Game-Based Security Definitions for Multi-Designated Verifier Signature Schemes}

We now introduce game-based correctness and security notions for MDVS schemes. For some security parameter $k$, we denote the advantage of an adversary $\mathbf{A}$ in 


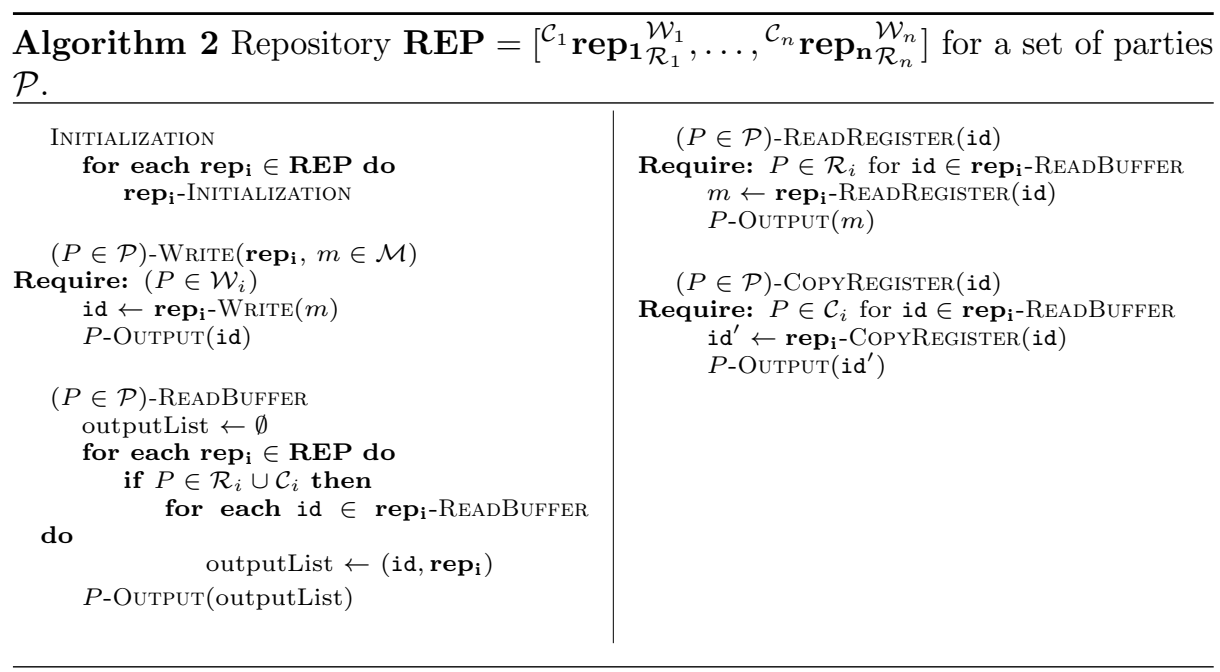

winning the game defined by some security notion $\mathrm{X}$ for an MDVS scheme $\Pi$ as $A d v_{k}^{\Pi-\mathrm{X}}(\mathbf{A})$. Whenever it is clear from the context, we simply write $A d v_{k}^{\mathrm{X}}(\mathbf{A})$, omitting $\Pi$ from the notation. In the literature, one typically makes statements about a sequence of adversaries $\overrightarrow{\mathbf{A}}=\left\{\mathbf{A}_{k}\right\}_{k \in \mathbb{N}}$, one for each security parameter, and the correctness and security of a scheme are defined asymptotically. More precisely, it is required that for any efficient adversary $\overrightarrow{\mathbf{A}}$ - where efficient usually means that $\overrightarrow{\mathbf{A}}$ is a non-uniform probabilistic polynomial time adversary - the function induced by the advantages of each $\mathbf{A}_{k}$ in winning the corresponding game for $k$ is at most negligible in $k$. But since the reductions in this paper are nonasymptotic - for any system $\mathbf{D}_{k}$ that can distinguish the real world from the ideal world we construct an adversary $\mathbf{A}_{k}$ that can win the corresponding game with comparable advantage - the asymptotic behavior of the games is mostly irrelevant for our results and is thus omitted for simplicity; it is rather straightforward to obtain the asymptotic security notions from the non-asymptotic ones we give below (alternatively, refer to [1] for the asymptotic definitions of the notions given ahead). As already mentioned, since most security statements are made for a fixed (but arbitrary) security parameter $k$, we usually omit it from the notation and simply write $A d v^{\mathrm{X}}(\mathbf{A})$ instead.

One can find multiple definitions of MDVS schemes in the literature 9 , 11, 16, 32. In this paper, we define an MDVS scheme $\Pi$ as a 5 -tuple $\Pi=$ (Setup, $G_{S}, G_{V}$, Sign, Vfy) of Probabilistic Polynomial Time algorithms (PPTs), following 17. Setup takes the security parameter as input, and produces public parameters (pp) and a master secret key (msk),

$$
(\mathrm{pp}, \mathrm{msk}) \leftarrow \operatorname{Setup}\left(1^{k}\right)
$$


These are then used by $G_{S}$ and $G_{V}$ to generate pairs of public and secret keys for the signers and verifiers, respectively,

$$
\begin{aligned}
& \left(\mathrm{spk}_{1}, \mathrm{ssk}_{1}\right) \leftarrow G_{S}(\mathrm{pp}, \mathrm{msk}), \quad \ldots \quad\left(\mathrm{spk}_{m}, \mathrm{ssk}_{m}\right) \leftarrow G_{S}(\mathrm{pp}, \mathrm{msk}), \\
& \left(\mathrm{vpk}_{1}, \mathrm{vsk}_{1}\right) \leftarrow G_{V}(\mathrm{pp}, \mathrm{msk}), \quad \ldots \quad\left(\mathrm{vpk}_{n}, \mathrm{vsk}_{n}\right) \leftarrow G_{V}(\mathrm{pp}, \mathrm{msk}) .
\end{aligned}
$$

Finally, the signing algorithm Sign requires the signer's secret key and the public keys of all the verifiers, and the verifying algorithm Vfy requires the signer's public key, the secret key of whoever is verifying and the public keys of all verifiers. For example suppose that party $A$ is signing a message $m$ for a set of verifiers $\mathcal{V}$ and that $B \in \mathcal{V}$ verifies the signature, then

$$
\begin{aligned}
\sigma & \leftarrow \operatorname{Sign}\left(\mathrm{pp}, \mathrm{ssk}_{A},\left\{\mathrm{vpk}_{i}\right\}_{i \in \mathcal{V}}, m\right) \\
b & \leftarrow \operatorname{Vfy}\left(\mathrm{pp}, \mathrm{spk}_{A}, \mathrm{vsk}_{B},\{\mathrm{vpk}\}_{i \in \mathcal{V}}, m, \sigma\right),
\end{aligned}
$$

where $b=1$ if the verification succeeds and $b=0$ otherwise.

The MDVS correctness and security notions given next are defined in terms of a game played between a challenger and an adversary. In game-based definitions an adversary $\mathbf{A}$ interacts with a game system $\mathbf{G}$ and tries winning the game. We denote this interaction by AG. At the end of the interaction, the game system $\mathbf{G}$ outputs a bit $b \in\{$ win, lose $\}$ that indicates whether $\mathbf{A}$ won the game. As we will see next, the definition of the adversary's advantage in winning a game is defined differently depending on the security notion. Typically a game system provides the adversary with a set of oracles that it can interact with to help winning the game. If game system $\mathbf{G}$ provides adversary $\mathbf{A}$ with access to oracles $\mathcal{O}_{1}$ and $\mathcal{O}_{2}$, the interaction between $\mathbf{A}$ and $\mathbf{G}$ can alternatively be denoted as $\mathbf{A}^{\mathcal{O}_{1}, \mathcal{O}_{2}}$, making explicit what capabilities $\mathbf{A}$ is given while playing the game. Before proceeding to the actual MDVS correctness security definitions, we first introduce some oracles that the game systems defined ahead use. These following oracles are defined for a game-system with (an implicitly defined) security parameter $k$.

Public Parameter Generation Oracle: $\mathcal{O}_{P P}$

1. If a query to this oracle has been previously performed then simply output the previously generated public parameters pp.

2. Otherwise, compute and store (pp, msk $) \leftarrow \operatorname{Setup}\left(1^{k}\right)$. Then, output pp.

Signer Key-Pair Generation Oracle: $\mathcal{O}_{S K}\left(A_{i}\right)$

1. If a query to this oracle has been previously performed for $A_{i}$, look up and return the previously generated key.

2. Otherwise, output and store $\left(\mathrm{spk}_{i}, \mathrm{ssk}_{i}\right) \leftarrow G_{S}$ (pp, msk).

Verifier Key-Pair Generation Oracle: $\mathcal{O}_{V K}\left(B_{j}\right)$

1. Analogous to the Signer Key-Pair Generation Oracle.

Signer Public-Key Oracle: $\mathcal{O}_{S P K}\left(A_{i}\right)$

1. $\left(\operatorname{spk}_{i}, \mathrm{ssk}_{i}\right) \leftarrow \mathcal{O}_{S K}\left(A_{i}\right)$.

2. Output $\mathrm{spk}_{i}$.

Verifier Public-Key Oracle: $\mathcal{O}_{V P K}\left(B_{j}\right)$

1. Analogous to the Signer Public-Key Oracle.

Signing Oracle: $\mathcal{O}_{S}\left(A_{i}, \mathcal{V}, m\right)$ 
1. $\left(\mathrm{spk}_{i}, \mathrm{ssk}_{i}\right) \leftarrow \mathcal{O}_{S K}\left(A_{i}\right)$.

2. For all $B_{j} \in \mathcal{V}: \operatorname{vpk}_{j} \leftarrow \mathcal{O}_{V P K}\left(B_{j}\right)$.

3. Output $\sigma \leftarrow \operatorname{Sign}\left(\mathrm{pp}, \mathbf{s s k}_{i},\left\{\operatorname{vpk}_{j}\right\}_{B_{j} \in \mathcal{V}}, m\right)$.

Verification Oracle: $\mathcal{O}_{V}\left(A_{i}, B_{j}, \mathcal{V}, m, \sigma\right)$

1. $\operatorname{spk}_{i} \leftarrow \mathcal{O}_{S P K}\left(A_{i}\right)$.

2. For all $B_{l} \in \mathcal{V}: \operatorname{vpk}_{l} \leftarrow \mathcal{O}_{V P K}\left(B_{l}\right)$.

3. $\left(\mathrm{vpk}_{j}, \mathrm{vsk}_{j}\right) \leftarrow \mathcal{O}_{V K}\left(B_{j}\right)$.

4. Output $d \leftarrow V f y\left(\mathrm{pp}, \mathrm{spk}_{i}, \mathrm{vsk}_{j},\left\{\mathrm{vpk}_{l}\right\}_{B_{l} \in \mathcal{V}}, m, \sigma\right)$.

We now introduce the relevant game-based notions for MDVS schemes. Let $\Pi=\left(\right.$ Setup $, G_{S}, G_{V}$, Sign, Vfy $)$ be an MDVS scheme with security parameter $k$.

Definition 2 (Correctness). Consider the following game played between an adversary $\mathbf{A}$ and the game system $\mathbf{G}{ }^{\text {Corr }}$ :

$$
\text { 1. } \mathbf{A}^{\mathcal{O}_{P P}, \mathcal{O}_{S K}, \mathcal{O}_{V K}, \mathcal{O}_{S P K}, \mathcal{O}_{V P K}, \mathcal{O}_{S}, \mathcal{O}_{V}}
$$

A wins the game if there are two queries $q_{S}$ and $q_{V}$ to $\mathcal{O}_{S}$ and $\mathcal{O}_{V}$, respectively, where $q_{S}$ has input $\left(A_{i}, \mathcal{V}, m\right)$ and $q_{V}$ has input $\left(A_{i}{ }^{\prime}, B_{j}, \mathcal{V}^{\prime}, m^{\prime}, \sigma\right)$, satisfying $A_{i}=A_{i}{ }^{\prime}, \mathcal{V}=\mathcal{V}^{\prime}, B_{j} \in \mathcal{V}$, the input $\sigma$ in $q_{V}$ is the output of $q_{S}$, the output of $q_{V}$ is 0 , and $\mathbf{A}$ did not previously query (i.e. before either $q_{S}$ or $\left.q_{V}\right) \mathcal{O}_{S K}$ on (input) $A_{i}$ nor $\mathcal{O}_{V K}$ on $B_{j}$.

We define the advantage of $\mathbf{A}$ in winning the correctness game as

$$
A d v^{\operatorname{Corr}}(\mathbf{A}):=\operatorname{Pr}\left[\mathbf{A G}^{\text {Corr }}=\text { win }\right] .
$$

The following security notions-Definition 3 and Definition 4 -correspond to multi-challenge variants of existing security notions from the literature 11 32 . It is worth mentioning that the multi-challenge versions of both of these security notions are asymptotically equivalent to the single challenge counterparts (meaning that if a scheme asymptotically satisfies the single-challenge version of either of these notions then it also asymptotically satisfies the corresponding multi-challenge version). To allow for the multiple challenges, we will introduce an additional oracle $\mathcal{O}_{\text {Challenge }}$ that adversaries use to submit the (possibly multiple) challenges to the games. Inputs to oracle $\mathcal{O}_{\text {Challenge }}$ are quadruples of the form $\left(m^{*}, A_{i}{ }^{*}, \mathcal{V}^{*}, \sigma^{*}\right)$; the oracle does not output any value. The exact behavior of the oracle, and in particular the definition of when the adversary wins the underlying game, depends on the security notion. On any query to this oracle, and regardless of whether it is a game-winning one, the oracle does not give any output.

The following security notion is the multi-challenge version of the Consistency security notion for MDVS, introduced by Damgård et al. in [11.

Definition 3 (Consistency). Consider the following game played between an adversary $\mathbf{A}$ and the game system $\mathbf{G}{ }^{\text {Cons }}$ :

$$
\text { 1. } \mathbf{A}^{\mathcal{O}_{P P}, \mathcal{O}_{S K}, \mathcal{O}_{V K}, \mathcal{O}_{S P K}, \mathcal{O}_{V P K}, \mathcal{O}_{S}, \mathcal{O}_{V}, \mathcal{O}_{\text {Challenge }}}
$$


where oracles $\mathcal{O}_{P P}, \mathcal{O}_{S K}, \mathcal{O}_{V K}, \mathcal{O}_{S P K}, \mathcal{O}_{V P K}, \mathcal{O}_{S}$ and $\mathcal{O}_{V}$ are as defined above,

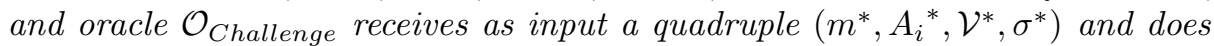
not give any output. We say that $\mathbf{A}$ wins the game if it queries $\mathcal{O}_{\text {Challenge }}$ on a quadruple $\left(m^{*}, A_{i}{ }^{*}, \mathcal{V}^{*}, \sigma^{*}\right)$ such that there exist verifiers $B_{j_{0}}, B_{j_{1}} \in \mathcal{V}^{*}$ such that:

$V f y\left(\mathrm{pp}, \mathrm{spk}, \mathrm{vsk}_{j_{0}},\left\{\mathrm{vpk}_{l}\right\}_{B_{l} \in \mathcal{V}^{*}}, m, \sigma\right) \neq V f y\left(\mathrm{pp}, \mathrm{spk}, \mathrm{vsk}_{j_{1}},\left\{\mathrm{vpk}_{l}\right\}_{B_{l} \in \mathcal{V}^{*}}, m, \sigma\right)$,

where all keys are the honestly generated outputs of the key generation oracles and $\mathcal{O}_{V K}$ was not queried on $B_{j_{0}}$ or $B_{j_{1}}$ at least until the query is submitted.

We define the advantage of $\mathbf{A}$ in winning the consistency security game as

$$
A d v^{\text {Cons }}(\mathbf{A}):=\operatorname{Pr}\left[\mathbf{A G}{ }^{\text {Cons }}=\text { win }\right] \text {. }
$$

The following notion can be seen as either the multi-challenge version of the unforgeability game introduced in [11], or the MDVS version of the unforgeability game for DSS from [3].

Definition 4 (Unforgeability). Consider the following game played between adversary $\mathbf{A}$ and game system $\mathbf{G}^{\text {Unforg }}$ :

$$
\text { 1. } \mathbf{A}^{\mathcal{O}_{P P}, \mathcal{O}_{S K}, \mathcal{O}_{V K}, \mathcal{O}_{S P K}, \mathcal{O}_{V P K}, \mathcal{O}_{S}, \mathcal{O}_{V}, \mathcal{O}_{\text {Challenge }}}
$$

where oracles $\mathcal{O}_{P P}, \mathcal{O}_{S K}, \mathcal{O}_{V K}, \mathcal{O}_{S P K}, \mathcal{O}_{V P K}, \mathcal{O}_{S}$ and $\mathcal{O}_{V}$ are as defined above,

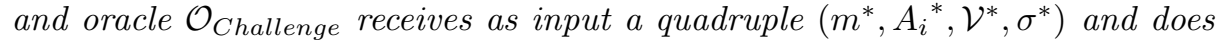
not give any output. We say that $\mathbf{A}$ wins the game if it makes a query, say $q$ to

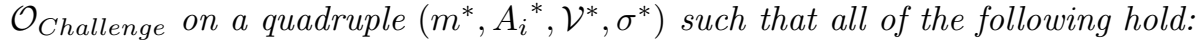

1. for all queries $A_{i}$ to $\mathcal{O}_{S K}$ until $q$ is issued, $A_{i}{ }^{*} \neq A_{i}$;

2. for all queries $\left(A_{i}, \mathcal{V}, m\right)$ issued to oracle $\mathcal{O}_{S}$ until $q$ was issued that result in a signature $\sigma$, it holds that $\left(A_{i}{ }^{*}, \mathcal{V}^{*}, m^{*}\right) \neq\left(A_{i}, \mathcal{V}, m\right)$;

3. there exists a verifier $B_{j^{\prime}} \in \mathcal{V}^{*}$ such that for all queries $B_{j}$ to oracle $\mathcal{O}_{V K}$ until $q$ was issued, $B_{j^{\prime}} \neq B_{j}$ and Vfy $\left(\mathrm{pp}, \mathrm{spk}_{i^{*}}, \mathrm{vsk}_{j^{\prime}},\left\{\mathrm{vpk}_{l}\right\}_{B_{l} \in \mathcal{V}^{*}}, m, \sigma\right)=1$, where all keys are honestly generated outputs of the key generation oracles.

We define the advantage of $\mathbf{A}$ in winning the unforgeability security game as

$$
A d v^{\text {Unforg }}(\mathbf{A}):=\operatorname{Pr}\left[\mathbf{A G}{ }^{\text {Unforg }}=\text { win }\right] .
$$

The following security notion is the multi-challenge variant of the Off-TheRecord (game-based) security notion introduced in 11. In contrast to the previous security notions, this new notion defines two game systems, $\mathbf{G}_{\mathbf{0}}^{\text {OTR-Forge }}$ and $\mathbf{G}_{\mathbf{1}}^{\text {OTR-Forge }}$, which are parameterized by an algorithm Forge. The game system also defines an oracle $\mathcal{O}_{\text {ChallengeSign }}$ whose behavior varies depending on the underlying game system, i.e. depending on $\mathbf{b} \in\{0,1\}$ the oracle $\mathcal{O}_{\text {ChallengeSign }}$ provided by $\mathbf{G}_{\mathbf{b}}^{\text {OTR-Forge }}$ behaves differently, as described below.

ChallengeSign Oracle: $\mathcal{O}_{\text {ChallengeSign }}\left(\right.$ type $\in\{$ sign, forge $\left.\}, m, A_{i}, \mathcal{V}, \mathcal{D}\right)$

For game system $\mathbf{G}_{\mathbf{b}}^{\text {OTR-Forge }}$, the oracle behaves as follows: 
1. $\left(\operatorname{spk}_{i}, \mathrm{ssk}_{i}\right) \leftarrow \mathcal{O}_{S K}\left(A_{i}\right)$.

2. For each $B_{j} \in \mathcal{V}: \operatorname{vpk}_{j} \leftarrow \mathcal{O}_{V P K}\left(B_{j}\right)$.

3. For each $B_{j} \in \mathcal{D}:\left(\operatorname{vpk}_{j}, \operatorname{vsk}_{j}\right) \leftarrow \mathcal{O}_{V K}\left(B_{j}\right)$.

4. $\sigma_{0} \leftarrow \Pi . \operatorname{Sign}\left(\mathrm{pp}, \mathrm{ssk}_{i},\left\{\mathrm{vpk}_{j}\right\}_{B_{j} \in \mathcal{V}}, m\right)$.

5. $\sigma_{\mathbf{1}} \leftarrow$ Forge $\left(\mathrm{pp}, \mathrm{spk}_{i},\left\{\mathrm{vpk}_{j}\right\}_{B_{j} \in \mathcal{V}},\left\{\mathrm{vsk}_{j}\right\}_{B_{j} \in \mathcal{D}}, m\right)$.

6. If $\mathbf{b}=0$, output $\sigma_{\mathbf{0}}$ if type $=\operatorname{sign}$ and $\sigma_{\mathbf{1}}$ if type $=$ forge.

7. If $\mathbf{b}=1$, output $\sigma_{\mathbf{1}}$.

Definition 5 (Off-The-Record). Consider an MDVS scheme $\Pi$ with $\Pi=$ (Setup, $G_{S}, G_{V}$, Sign, Vfy). In the following, let Forge be a PPT algorithm that on input $\mathrm{pp}, \mathrm{spk}_{i^{*}},\left\{\mathrm{vpk}_{l}\right\}_{B_{l} \in \mathcal{V}^{*}},\left\{\mathrm{vsk}_{j}\right\}_{B_{j} \in \mathcal{D}^{*}}$, and $m^{*}$ outputs a forged signature $\sigma^{\prime}$. For $\mathbf{b} \in\{0,1\}$, consider the following game played between an adversary $\mathbf{A}$ and game system $\mathbf{G}_{\mathbf{b}}^{\text {OTR-Forge }}$ :

1. $b^{\prime} \leftarrow \mathbf{A}^{\mathcal{O}_{P P}, \mathcal{O}_{S K}, \mathcal{O}_{V K}, \mathcal{O}_{S P K}, \mathcal{O}_{V P K}, \mathcal{O}_{V}, \mathcal{O}_{\text {ChallengeSign }}}$

A wins the game if $b^{\prime}=\mathbf{b}$ and for every query (type, $\left.m, A_{i}, \mathcal{V}, \mathcal{D}\right)$ to $\mathcal{O}_{\text {ChallengeSign }}$ all of the following hold:

1. $\mathcal{D} \subseteq \mathcal{V}$

2. for every query $B_{j}$ to $\mathcal{O}_{V K}, B_{j} \notin \mathcal{V} \backslash \mathcal{D}$;

3. for every query $A_{l}$ to $\mathcal{O}_{S K}, A_{l} \neq A_{i}$; and

4. letting $\sigma$ denote the output of $\mathcal{O}_{\text {ChallengeSign }}$ to the query above, for all queries $\left(A_{l}, B_{j}, \mathcal{V}^{\prime}, m^{\prime}, \sigma^{\prime}\right)$ to oracle $\mathcal{O}_{V}, \sigma^{\prime} \neq \sigma$.

We define the advantage of $\mathbf{A}$ in winning the Off-The-Record security game with respect to algorithm Forge as

$$
A d v^{\text {OTR-Forge }}(\mathbf{A}):=\mid \operatorname{Pr}\left[\mathbf{A G}_{\mathbf{0}}^{\text {OTR-Forge }}=\text { win }\right]+\operatorname{Pr}\left[\mathbf{A G}_{\mathbf{1}}^{\text {OTR-Forge }}=\text { win }\right]-1 \mid .
$$

\section{Modeling MDVS with Fixed Sender and Receivers}

In this section we consider a fixed sender $A$, a fixed set of receivers $\mathcal{R}=$ $\left\{B_{1}, \ldots, B_{n}\right\}$ and one eavesdropper $E$ that is neither sender nor receiver, and is always dishonest. The set of parties is then given by $\mathcal{P}=\left\{A, B_{1}, \ldots, B_{n}, E\right\}$. Furthermore, we assume that sender $A$ always designates $\mathcal{R}$ as the set of designated receivers for the messages it sends. This means in particular that if all receivers are honest then $E$ always learns when $A$ sends a message (as no other party can send messages).

\subsection{Real-World}

To communicate, each party in $\mathcal{P}$ has access to an insecure repository INS := $\mathbf{I N S}_{k}$ (for a fixed security parameter $k$ ) to which everyone can read from and write to (recall Sect. 2.3). In addition, parties also have access to a Key Generation 
Authority (KGA), which generates and stores the parties' key pairs ${ }^{10}$ For a fixed security parameter $k$, the KGA $:=\mathbf{K G A}_{k}$ resource runs the Setup algorithm giving it the (implicit) parameter $k$, and then generates and stores all key pairs for the sender $A$ and each receiver in $\mathcal{R}$, using $G_{S}$ and $G_{V}$, respectively. Every honest party can then query their own public-secret key pair, the public parameters and everyone's public key at their own interface. Dishonest parties can additionally query the public-secret key pairs of any other dishonest party. The semantics of the KGA resource is defined in Algorithm $3{ }^{11}$

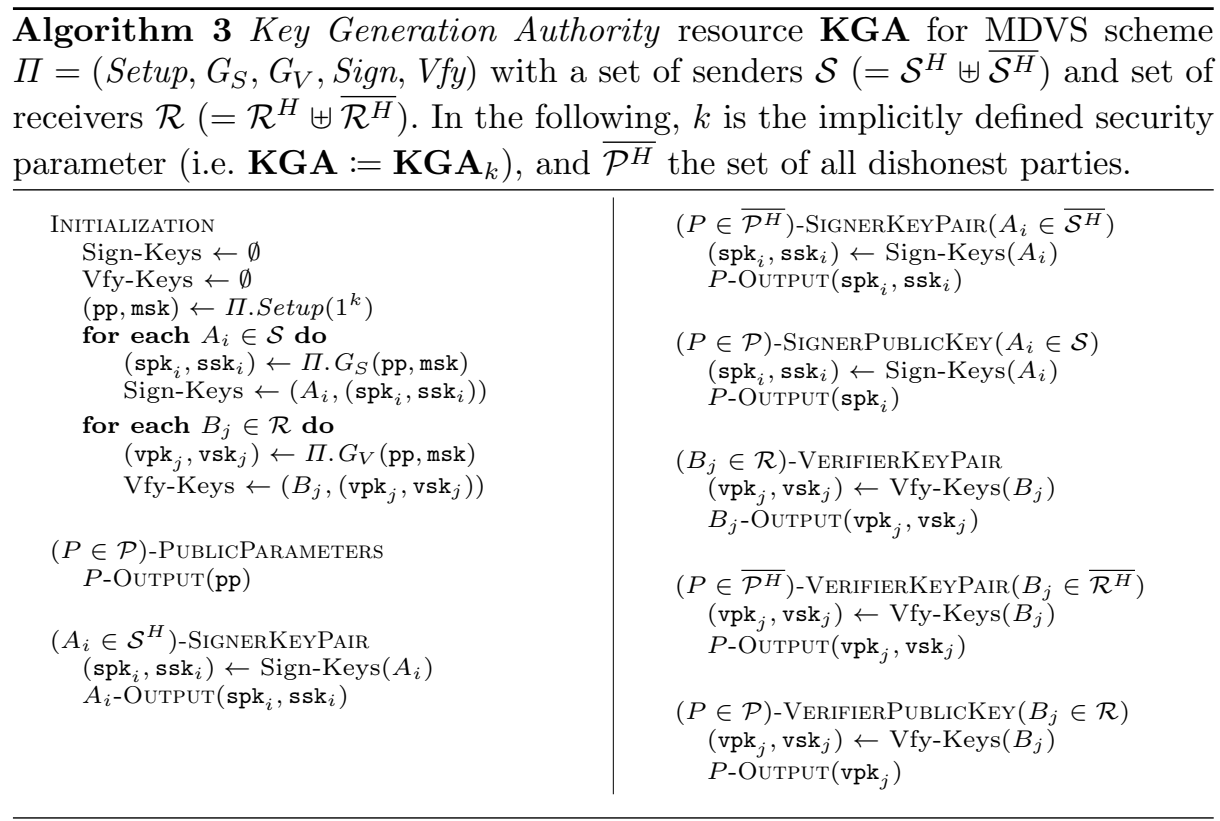

The sender $A$ runs a converter Snd (locally) and each receiver $B_{j} \in \mathcal{R}$ runs a converter Rcv (also locally). This means sender $A$ can send messages by simply running its converter Snd, and each receiver can receive messages by simply running its converter Rcv.

The Snd converter connects to INS and KGA at its inner interface, and has an outer interface that is identical to the interface of a repository for a party who is a writer, i.e. it provides a procedure WRITE which takes as input a label $\left\langle A_{i} \rightarrow \mathcal{V}\right\rangle$ defining the sender $A_{i}$ and set of receivers $\mathcal{V}$ and a message $m \in \mathcal{M}$ to be signed. Snd then gets the necessary keys and public parameters from KGA, signs the

10 The purpose of having an explicit KGA resource is guaranteeing that receivers know their secret keys, which is crucial for being able to achieve the exclusiveness of authenticity guarantee of MDVS schemes 13,29].

11 Algorithm 3 defines the behavior of KGA in the case of multiple senders, which will only be used in Sect. 5 
input message $m$ using the algorithm Sign, which outputs some signature $\sigma \in \mathcal{S}$, and then writes $\left(m, \sigma,\left(A_{i}, \mathcal{V}\right)\right)$ into the insecure repository INS. For simplicity, since in this section the label is always $\langle A \rightarrow \mathcal{R}\rangle$ it is simply omitted. In addition, rather than making the Snd converter always write $(m, \sigma,(A, \mathcal{R}))$ tuples into INS, we omit $(A, \mathcal{R})$ and simply write $(m, \sigma)$ pairs instead. The exact (simplified) semantics for converter Snd is given in Algorithm 4.

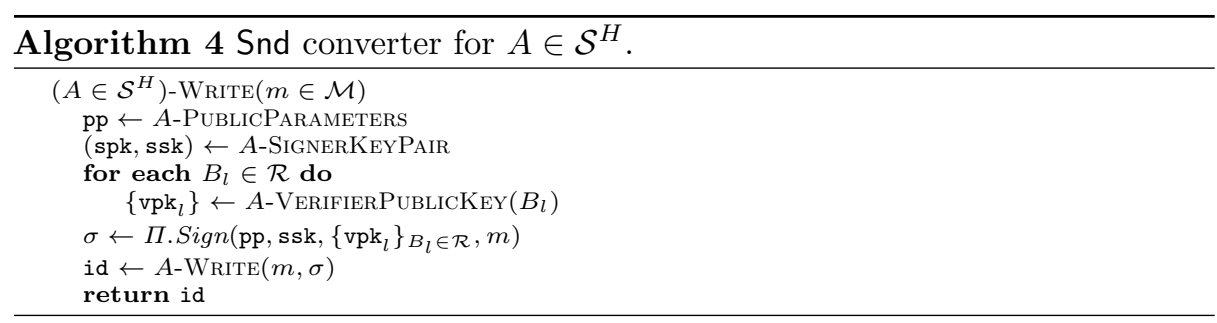

Similarly to Snd, the Rcv converter connects to KGA and INS at its inner interfaces and provides the same outer interface as a repository for a party with read access, i.e. it gives access to two read operations, namely READBUFFER and READREgister. The behavior of Rcv for each such read operation is specified by means of a procedure with the same name (i.e. a READBUFFER and a READREgister procedure). The READBUfFER procedure first reads all tuples $\left(m, \sigma,\left(A_{i}, \mathcal{V}\right)\right)$ written into INS - by issuing a READBuffer operation to INS followed by a series of READREGISTER operations, one for each id returned by the first operation - and for each tuple satisfying $A_{i}=A$ and $\mathcal{V}=\mathcal{R}$, the converter verifies whether $\sigma$ is a valid signature on $m$ with respect to sender $A$ and set of receivers $\mathcal{R}$. To this end, the Rcv converter first fetches all the public parameters and keys needed from KGA, and then checks if $\sigma$ is a valid signature on $m$ with respect to the public keys of the sender $A$ and of each receiver in $\mathcal{R}$ using the $V f y$ algorithm defined by the underlying MDVS scheme $\Pi$. The converter then outputs a list of pairs - one for each register stored in INS containing a valid message-signature pair according to Vfy and with respect to $A$ and $\mathcal{R}$-where each pair contains a register's id and a label $\langle A \rightarrow \mathcal{R}\rangle$. Since in this section the label is always the same, we simply omit it. The READREGISTER procedure of the Rcv converter receives as input the id of the register to be read; if the register contains a valid tuple (in the same sense as above) the procedure then outputs the message contained in the register. The exact (simplified) semantics for the Rcv converter is given in Algorithm 5.

In the case where the sender and all receivers are honest-i.e. $\mathcal{P}^{H}=\{A\} \cup \mathcal{R}^{H}$ with $\mathcal{R}^{H}=\mathcal{R}$ - the real world specification is given by

$$
\operatorname{Snd}^{A} \operatorname{Rcv}^{\mathcal{R}^{H}}\{[\text { KGA }, \mathbf{I N S}]\},
$$

where $\operatorname{Rcv}^{\mathcal{R}^{H}}=\operatorname{Rcv}^{B_{1}} \ldots \operatorname{Rcv}^{B_{n}}$ denotes all receiver converters run at the interfaces of $B_{j} \in \mathcal{R}^{H}$. This is illustrated in Fig. 1. As explained in Remark 3 in 


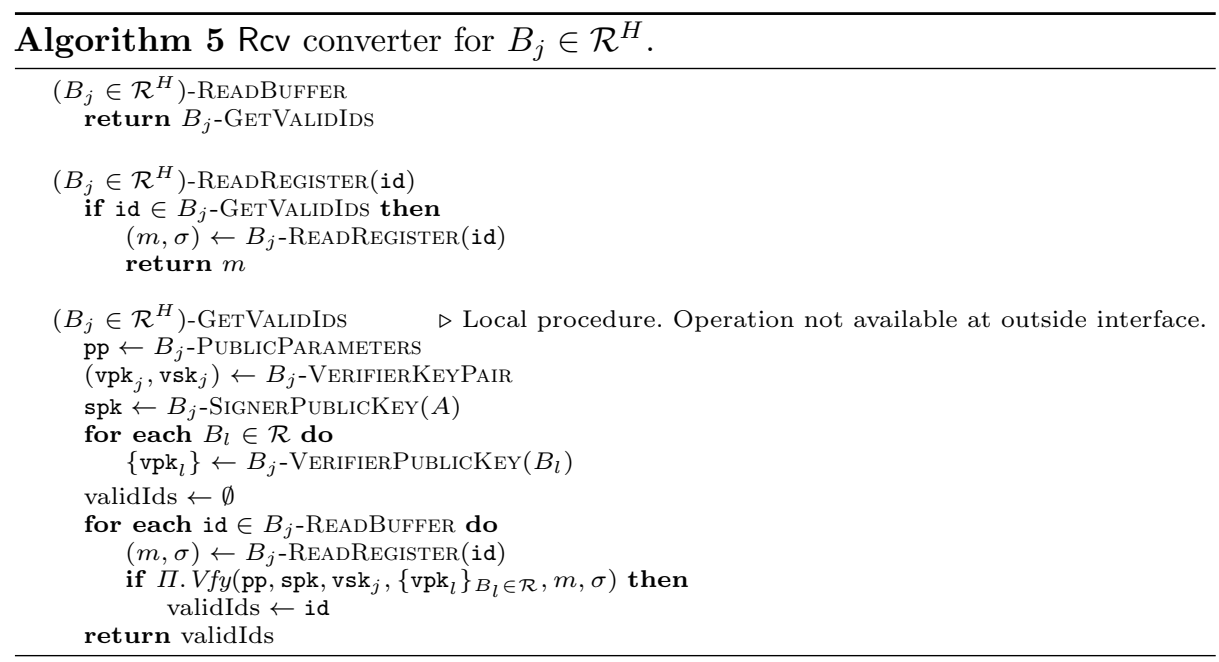

Sect. 2.2 if a party $P$ is dishonest, then we simply remove their converter from Eq. (4.1) to get the corresponding real world.

\subsection{Ideal-Worlds}

Whether the sender is honest or dishonest completely changes the guarantees one wishes to give, and thus completely changes the ideal world. So we divide this in two subsections, the first models a dishonest sender and the second an honest sender. Recall that the third-party $E$ is always dishonest.

Dishonest Sender. In case of a dishonest sender the only property the construction must capture is consistency, namely that all honest receivers in $\mathcal{R}^{H}$ get the same messages (for any $\mathcal{R}^{H} \neq \emptyset$ ). This means that even if all dishonest parties collude, including the sender $A$, the dishonest receivers $\overline{\mathcal{R}^{H}}$ and the third-party $E$, they are unable to generate confusion within the honest senders as to whether some message is authentic or not: either every receiver $B_{j} \in \mathcal{R}^{H}$ accepts a message as authentic or none does. A repository to which all honest receivers have read access captures this guarantee. Since dishonest parties may share secret keys with each other, any of them may have either read or write access. The repository we want to construct is then

$$
\langle A \rightarrow \mathcal{R}\rangle_{\mathcal{R} \cup\{A, E\}}^{\overline{\mathcal{R}^{H}} \cup\{A, E\}}
$$

where we have used $\langle A \rightarrow \mathcal{R}\rangle$ as label to denote the repository. By considering a set of converters $\Omega^{12}$ that could be run jointly at the dishonest parties' interfaces,

${ }^{12}$ We do not define $\Omega$ at this point, since in a finite setting there is no "good" and "bad" system (efficient or inefficient, negligible or non-negligible). Instead, in the theorem statement for a security proof we explicitly give the set $\Omega$ which is used, as the meaningfulness of the theorem will depend on the choice of this set. 


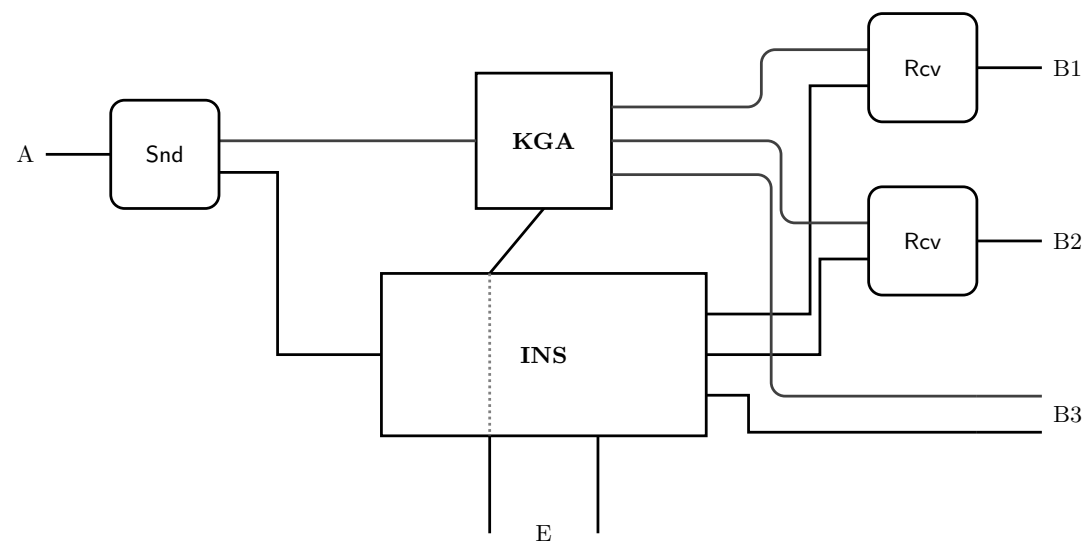

Fig. 1. Illustration of the real world system specified by Eq. (4.1) for the case where $\mathcal{R}=\{B 1, B 2, B 3\}$, with $\mathcal{R}^{H}=\{B 1, B 2\}$.

one can then define the ideal world specification $\mathcal{C}_{\Omega}^{\text {Fix }}$ capturing consistency as

$$
\mathcal{C}_{\Omega}^{\text {Fix }}:=\left\{\operatorname{sim}^{\overline{\mathcal{R}^{H}} \cup\{A, E\}}\left[\langle A \rightarrow \mathcal{R}\rangle_{\mathcal{R} \cup\{A, E\}}^{\overline{\mathcal{R}^{H}} \cup\{A, E\}}\right]\right\}_{\text {sim } \in \Omega} .
$$

Finally, we also want the ideal world to contain systems that are indistinguishable from the perfect ones defined above, so we put an $\varepsilon$-ball around the ideal resource 13 The ideal world is then

$$
\left(\mathcal{C}_{\Omega}^{\mathrm{Fix}}\right)^{\varepsilon}
$$

Honest Sender. In the case of an honest sender, there are two properties that we expect from an MDVS scheme. The first is that the (honest) designated receivers can verify the authenticity of the message as coming from the actual sender $A$. The second is that this authenticity is exclusive to the designated receivers ${ }^{14}$ i.e. a third party $E$ cannot be convinced that any message was sent by $A$, even if dishonest receivers leak all their secret keys to $E{ }^{15}$ To this end, MDVS schemes need to be such that every possible set of dishonest receivers can (cooperatively) come up with forged signatures that are indistinguishable from the real ones generated by $A$ to the third-party $E$ (who has access to the

${ }^{13}$ Like for $\Omega$ (see Footnote 12 we do not define acceptable $\varepsilon$ here, but in a theorem statement for a security proof we explicitly give the one used.

${ }^{14}$ A third important property is correctness, but in our setting dishonest parties cannot delete the messages of honest parties, so correctness follows from authenticity and does not need to be considered separately.

${ }^{15}$ If all receivers are honest only $A$ can send messages, and so in this case $E$ just knows that $A$ must be the one sending messages. 


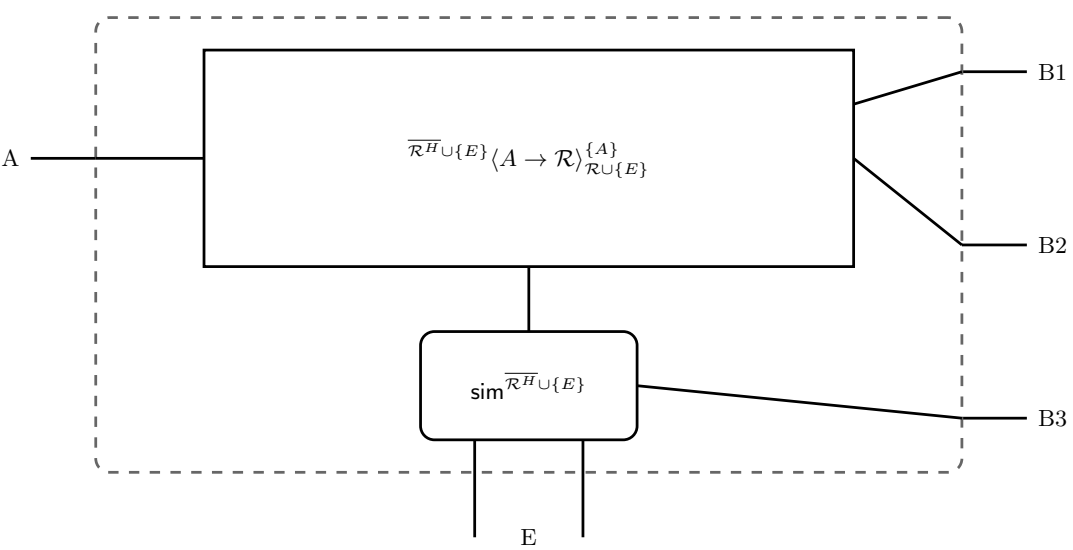

Fig. 2. Illustration of an ideal world system from the $\mathcal{A}_{\Omega}^{\text {Fix }}$ specification Eq. (4.3) for the case where $\mathcal{R}=\{B 1, B 2, B 3\}$, with $\mathcal{R}^{H}=\{B 1, B 2\}$.

dishonest receivers' secret keys). Note, on the other hand, that honest designated receivers are not "fooled" by signatures forged by dishonest (designated) receivers; authenticity guarantees that honest designated receivers can verify whether it was really $A$ signing a message or otherwise.

Authenticity is straightforward to capture: it essentially corresponds to a repository where only the sender can write, but everyone else can read. The only twist is that dishonest parties might be able to duplicate messages written by the sender $A[3]{ }^{16}$ So the repository we wish to be constructed is given by

$$
\overline{\mathcal{R}^{H}} \cup\{E\}\langle A \rightarrow \mathcal{R}\rangle_{\mathcal{R} \cup\{E\}}^{\{A\}} .
$$

As for consistency, by considering a set of converters $\Omega$ that could be run jointly at the dishonest parties' interfaces, one can then define the ideal world specification $\mathcal{A}_{\Omega}^{\text {Fix }}$ capturing authenticity as

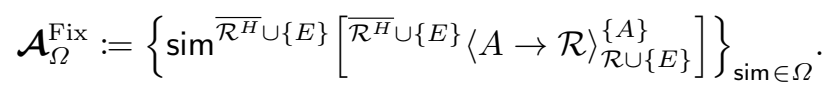

Here too, we extend the ideal world to also contain systems that are indistinguishable from those in Eq. (4.3) by adding a $\varepsilon$-ball around the specification. The final ideal specification is thus

$$
\left(\mathcal{A}_{\Omega}^{\text {Fix }}\right)^{\varepsilon}
$$

Fig. 2 illustrates the ideal world systems from the $\mathcal{A}_{\Omega}^{\text {Fix }}$ specification.

${ }^{16}$ They can do this either by creating a copy of a valid message-signature pair or by sending the same message but with a different signature. 
Finally, the notion of exclusiveness of authenticity is captured in a world where there exists an (explicit) behavior $\pi$ for the dishonest receivers that allows them to generate signatures that look just like fresh signatures to any third party $E$. This means that running $\pi$ would result in a repository in which both the honest sender $A$ and all the dishonest receivers in $\overline{\mathcal{R}^{H}}$ can write and $E$ can read, namely ${ }^{17}$

$$
\langle A \rightarrow \mathcal{R}\rangle_{\{E\}}^{\{A\} \cup \overline{\mathcal{R}^{H}}} .
$$

As usual, we extend the specification by attaching a converter sim at the dishonest parties' interfaces. However, sim is not allowed to block or cover the write ability at the interfaces of the parties in $\overline{\mathcal{R}^{H}}$, because we wish to guarantee that a dishonest receiver can write to the repository ${ }^{18}$ The specification providing the guarantee that $E$ cannot distinguish real signatures (created by $A$ ) from fake ones (forged by the dishonest designated receivers) is given by

$$
\widehat{\mathcal{X}}_{\Omega}^{\text {Fix }}:=\left\{\operatorname{sim}^{\{E\}}\left[\langle A \rightarrow \mathcal{R}\rangle_{\{E\}}^{\{A\} \cup \overline{\mathcal{R}^{H}}}\right]\right\}_{\operatorname{sim} \in \Omega} .
$$

Fig. 3 illustrates an ideal world system from $\widehat{\mathcal{X}}_{\Omega}^{\text {Fix }}$. As stated above, there must exist a converter $\pi$ that the dishonest receivers $\overline{\mathcal{R}^{H}}$ can run jointly to achieve a resource in the specification from Eq. (4.5). Since dishonest receivers could have run (and can run) $\pi$, a third party $E$ cannot tell if the message was sent by them or by the honest sender $A$ even when given access to the keys of all dishonest receivers (notice that $E$, being one of the dishonest parties, can query the KGA to obtain the secret keys of any dishonest receiver). Putting things together, the ideal world is defined as

$$
\mathcal{X}_{\Omega, \pi}^{\mathrm{Fix}}:=\left\{\mathbf{V}: \pi^{\overline{\mathcal{R}^{H}}} \perp^{\mathcal{R}^{H}} \mathbf{V} \in \widehat{\mathcal{X}}_{\Omega}^{\mathrm{Fix}}\right\}
$$

where $\perp^{\mathcal{R}^{H}}$ blocks the interfaces of all honest receivers $\mathcal{R}^{H} 19$ Fig. 4 illustrates a possible real world system in the $\mathcal{X}_{\Omega, \pi}^{\text {Fix }}$ specification with a converter $\perp^{\mathcal{R}^{H}}$ blocking the interface of the (only) honest receiver $B_{1}$, and protocol $\pi^{\overline{\mathcal{R}^{H}}}$ attached to the interfaces of the dishonest receivers (i.e. $B_{2}$ and $B_{3}$ ). Again, we put an $\varepsilon$-ball around Eq. (4.6), and define the ideal specification for the exclusiveness of authenticity to be

$$
\left(\mathcal{X}_{\Omega, \pi}^{\text {Fix }}\right)^{\varepsilon}
$$

$\overline{17}$ As one might note, the repository in Eq. (4.4) does not allow the honest designated receivers $\mathcal{R}^{H}$ to read. The reason for this is that the security statement does not concern them, so we remove them from the security statement. In fact, due to authenticity the honest designated receivers could distinguish signatures written by Alice or forged by the dishonest receivers.

18 Traditional composable security frameworks require the simulator to cover all dishonest interfaces making it impossible to model Eq. (4.5)

${ }^{19}$ Note that the ideal specification in Eq. (4.6) does not follow the ideal-functionalitysimulator paradigm, making it impossible to (directly) model the same thing in traditional composable frameworks. 


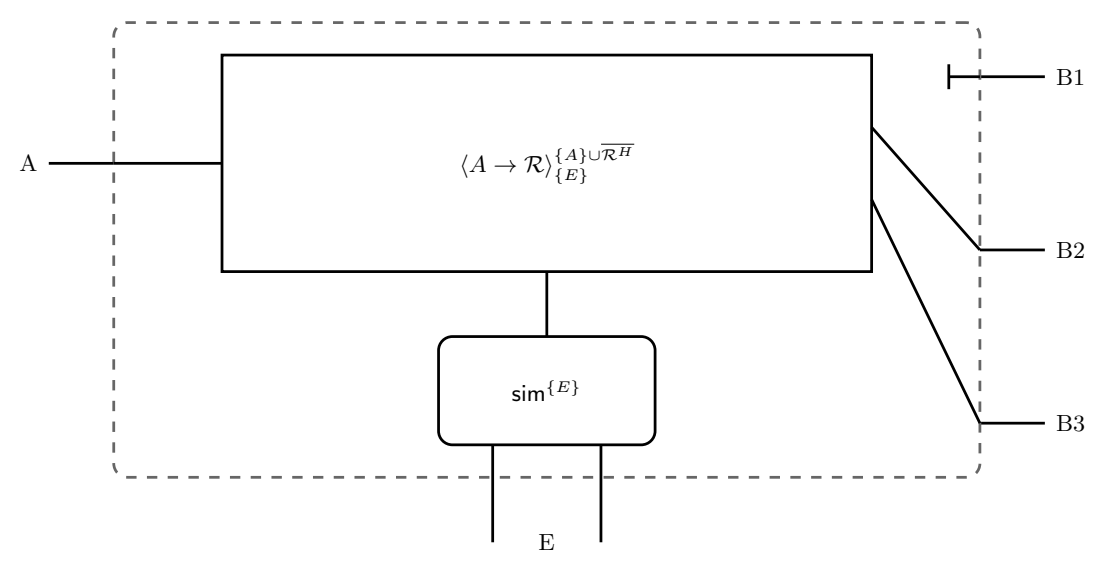

Fig. 3. Illustration of an ideal world system from the $\widehat{\mathcal{X}}_{\Omega}^{\text {Fix }}$ specification $[$ Eq. (4.5) for the case where $\mathcal{R}=\{B 1, B 2, B 3\}$, with $\mathcal{R}^{H}=\{B 1\}$.

Putting things together, the ideal world specification for the case of an honest sender is then given by

$$
\mathcal{S}=\left(\mathcal{A}_{\Omega}^{\mathrm{Fix}}\right)^{\varepsilon} \cap\left(\mathcal{X}_{\Omega^{\prime}, \pi}^{\mathrm{Fix}}\right)^{\varepsilon^{\prime}}
$$

\subsection{Reduction to Game-Based Security}

We now compare our composable notions against the existing game-based security notions from the literature (given in Sect. 3).

The first theorem shows that in the case of a dishonest sender, the advantage in distinguishing the real and ideal systems is upper bounded by the advantage in winning the consistency game.

Theorem 1. When the sender $\mathcal{A}$ is dishonest, i.e. $\mathcal{P}^{H}=\mathcal{R}^{H}$, we find an explicit reduction system $\mathbf{C}$ (defined in Algorithm 12) and an explicit simulator sim (defined in Algorithm 11) such that for any $\Omega \supseteq\{\operatorname{sim}\}$ :

$$
\mathcal{R} \subseteq\left(\mathcal{C}_{\Omega}^{\mathrm{Fix}}\right)^{A d v^{\text {Cons }}(\cdot \mathbf{C})}
$$

where for any distinguisher $\mathbf{D}, A d v^{\text {Cons }}(\mathbf{D C})$ is the advantage of $\mathbf{D}^{\prime}=\mathbf{D C}$ (the distinguisher resulting from composing $\mathbf{D}$ and $\mathbf{C}$ ) in winning the Consistency game Definition 3.).

A proof of Theorem 1 is provided in Appendix A.1.

The second theorem shows that in the case of an honest sender, the advantage in distinguishing the real world from the ideal world for authenticity is upper bounded by the advantage in winning the unforgeability game and the correctness game. 


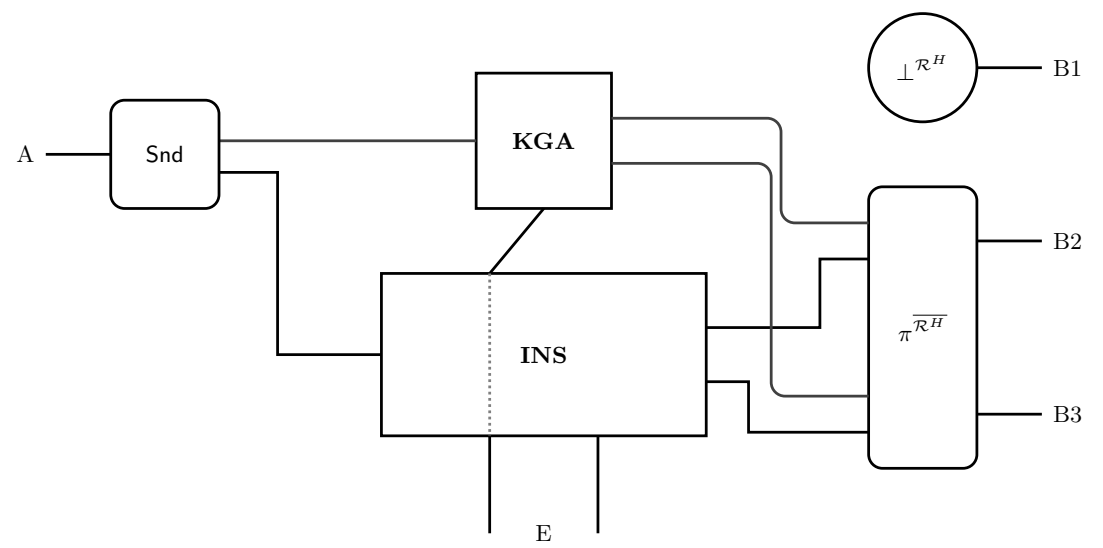

Fig. 4. Illustration of a possible real world system in the $\boldsymbol{X}_{\Omega, \pi}^{\text {Fix }}$ specification Eq. (4.6) for the case where $\mathcal{R}=\{B 1, B 2, B 3\}$, with $\mathcal{R}^{H}=\{B 1\}$. Converter $\perp^{\mathcal{R}^{H}}$ blocks $B_{1}$ 's interface; signature forgery protocol $\pi^{\overline{\mathcal{R}^{H}}}$ is attached to the interfaces of $B_{2}$ and $B_{3}$.

Theorem 2. When the sender is honest, i.e. for $\mathcal{P}^{H}=\{A\} \cup \mathcal{R}^{H}$, we find explicit reduction systems $\mathbf{C}^{\prime}$ and $\mathbf{C}$ (the latter is defined in Algorithm 14) and an explicit simulator sim (defined in Algorithm 13) such that for any $\Omega \supseteq\{\operatorname{sim}\}$ :

$$
\boldsymbol{R} \subseteq\left(\mathcal{A}_{\Omega}^{\mathrm{Fix}}\right)^{A d v^{\text {Unforg }}(\cdot \mathbf{C})+A d v^{\text {Corr }}\left(\cdot \mathbf{C}^{\prime}\right)}
$$

where for any distinguisher $\mathbf{D}, A d v^{\text {Unforg }}(\mathbf{D C})$ is the advantage of $\mathbf{D}^{\prime}=\mathbf{D C}$ (the distinguisher resulting from composing $\mathbf{D}$ and $\mathbf{C}$ ) in winning the Unforgeability game (see Definition 4), and $A d v^{C o r r}(\mathbf{D C})$ is the advantage of $\mathbf{D}^{\prime \prime}=\mathbf{D} \mathbf{C}^{\prime}$ in winning the Correctness game (see Definition 2).

A proof of Theorem 2 is provided in Appendix A.2.

In the third theorem we show that in the case of an honest sender, the advantage in distinguishing the real world from the ideal world for the exclusiveness of authenticity is bounded by the advantage in winning the Off-The-Record game.

Theorem 3. When the sender is honest, i.e. for $\mathcal{P}^{H}=\{A\} \cup \mathcal{R}^{H}$, and for any signature forgery algorithm Forge suitable for the Off-The-Record security notion (see Definition 5), we find an explicit reduction system $\mathbf{C}$ (defined in Algorithm 16) and an explicit simulator sim (defined in Algorithm 15) such that for any $\Omega \supseteq\{\operatorname{sim}\}$ :

$$
\mathcal{R} \subseteq\left(\mathcal{X}_{\Omega, \pi \text { Forge }}^{\text {Fix }}\right)^{\text {Adv }^{\text {OTR-Forge }}(\cdot \mathbf{C})},
$$

where $\pi^{\text {Forge }}$ is the converter running the Forge algorithm (see Algorithm 6 ), and for any distinguisher $\mathbf{D}, A d v^{\text {OTR-Forge }}(\mathbf{D C})$ is the advantage of $\mathbf{D}^{\prime}=\mathbf{D C}$ (the distinguisher resulting from composing $\mathbf{D}$ and $\mathbf{C})$ in winning the Off-The-Record game with respect to the signature forgery algorithm Forge (see Definition 5).

A proof of Theorem 3 is provided in Appendix A.3 


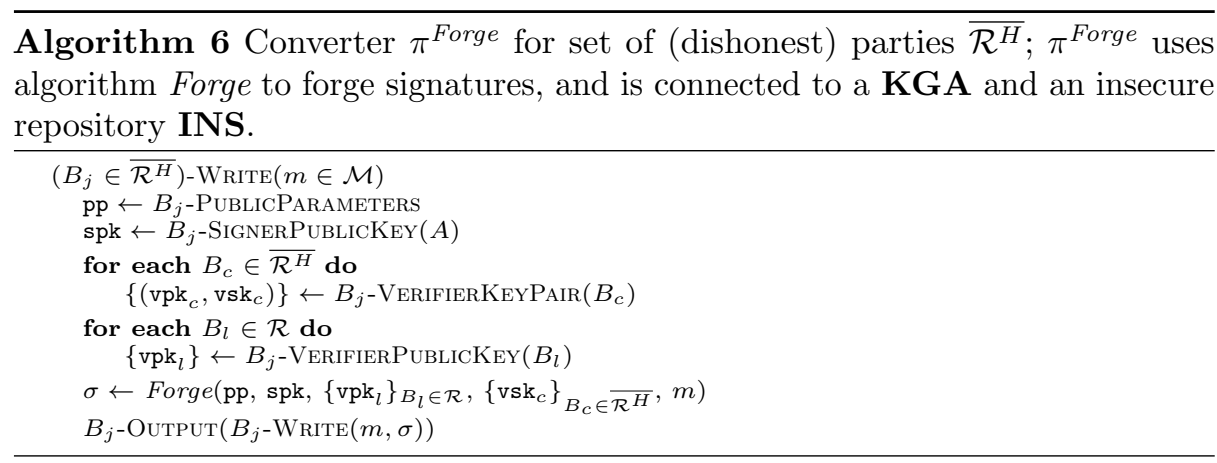

\section{Modeling MDVS for Arbitrary Parties}

In this section we model the security of MDVS schemes in the presence of multiple possible senders and multiple sets of receivers, which corresponds to a generalization of the models given in Sect. 4. Throughout this section, we denote by $\mathcal{S}$ the set of senders, and by $\mathcal{S}^{H}$ and $\mathcal{S}^{H}$ the partitions of $\mathcal{S}$ corresponding to honest and dishonest senders. As before, $\mathcal{R}, \mathcal{R}^{H}$ and $\overline{\mathcal{R}^{H}}$ correspond to the set of all receivers, honest and dishonest receivers, respectively. Furthermore, we assume that $\mathcal{R}^{H}, \overline{\mathcal{R}^{H}}, \mathcal{S}^{H}$ and $\overline{\mathcal{S}^{H}}$ are all non-empty sets.

\section{$5.1 \quad$ Real-World}

The real world specification for this security model is similar to the one given in Sect. 4.1 for the fixed sender and fixed set of receivers case. However, in Sect. 4 we made a few simplifications in the description of converters Snd and Rcv namely, the fixed sender and a fixed set of receiver are hard-coded in the converters. In this section, the converters Snd ${ }^{A r b}$ and Rcv ${ }^{A r b}$ (see Algorithm 7 and Algorithm 8, respectively) allow the sender to specify the set of receivers for each message they send, and the Rcv ${ }^{\text {Arb }}$ converters explicitly output the sender and the set of designated receivers. Moreover, the Snd ${ }^{\text {Arb }}$ converter now attaches to each message-signature pair also the sender and set of receivers meant for that message-signature pair; the Rcv ${ }^{A r b}$ converter then relies on this information to validate the authenticity of messages meant for the corresponding receiver. Apart from this, the real-world specification is as before: the Snd ${ }^{A r b}$ and Rcv ${ }^{A r b}$ converters connect to the KGA and to an insecure repository INS, and behave otherwise similarly to the Snd and Rcv converters. Since we assumed that $\mathcal{S}^{H}$ and $\mathcal{R}^{H}$ are non-empty sets, the real-world specification is then defined by

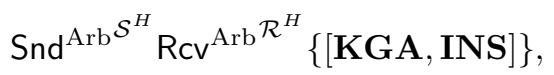

as illustrated in Fig. 5. 

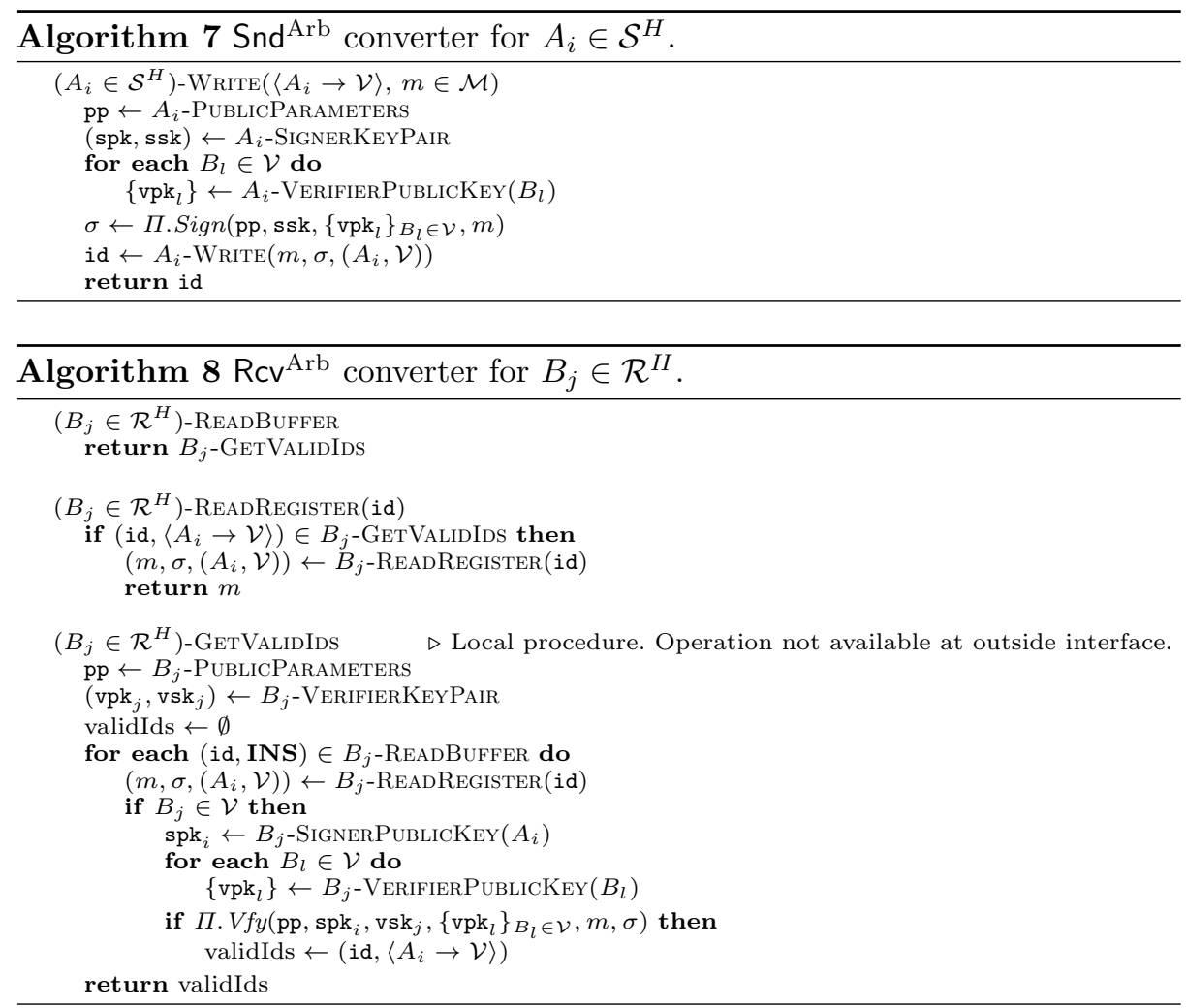

\subsection{Ideal-Worlds}

As aforementioned in Sect. 4.2, the guarantees given by the ideal world when a sender is honest are completely different from the ones when it is dishonest. However, since now we have both honest and dishonest senders at the same time, the ideal-world specification modeling the security of MDVS schemes consists of the intersection of only two (relaxed) specifications, one capturing the consistency and authenticity together $(\mathcal{C A})_{\Omega}^{\mathrm{Arb}}{ }^{20}$ and one capturing the exclusiveness of authenticity $\mathcal{X}_{\Omega^{\prime}, \pi}^{\mathrm{Arb}}$. The ideal world is then

$$
\mathcal{S}=\left((\mathcal{C} \mathcal{A})_{\Omega}^{\mathrm{Arb}}\right)^{\varepsilon} \cap\left(\mathcal{X}_{\Omega^{\prime}, \pi}^{\mathrm{Arb}}\right)^{\varepsilon^{\prime}}
$$

One key difference between the model we now introduce and the one from Sect. 4 is that we may have dishonest parties (other than Eve) that are neither sender nor designated receivers in this section, and we require exclusiveness of authenticity to hold with respect to them as well. So it is not sufficient that (any

${ }^{20}$ As noted in Sect. 4 in our setting correctness follows from authenticity, so it does not need to be considered separately. 


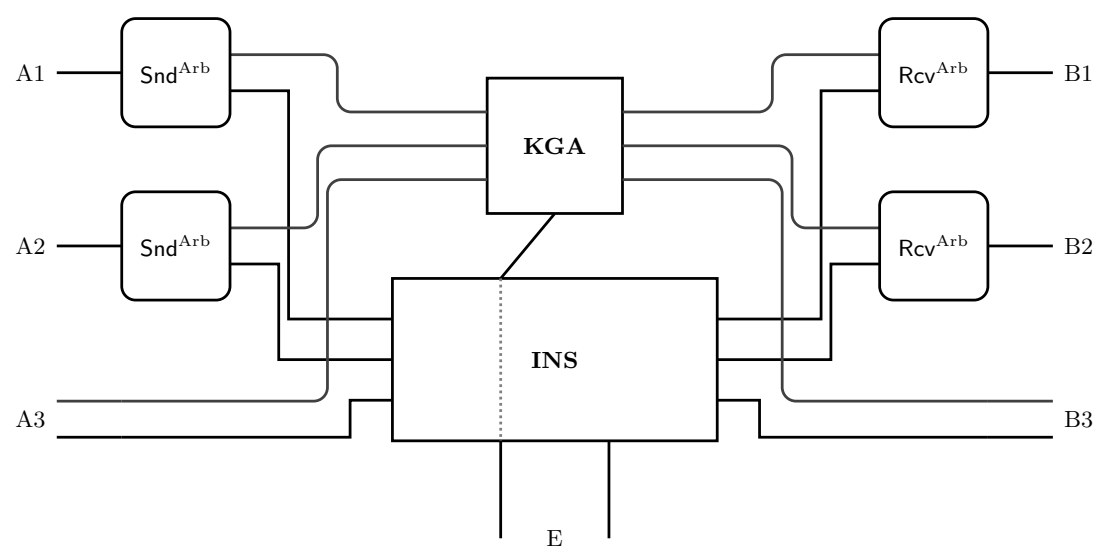

Fig. 5. Illustration of the real world system specified by Eq. (5.1) for the case where $\mathcal{S}=\{A 1, A 2, A 3\}$ and $\mathcal{R}=\{B 1, B 2, B 3\}$, with $\mathcal{S}^{H}=\{A 1, A 2\}$ and $\mathcal{R}^{H}=\{B 1, B 2\}$.

non-empty subset of) dishonest verifiers who have a secret verification key can forge signatures, parties with no secret verification key should also be able to forge 21

Consistency and Authenticity. As just mentioned, $(\mathcal{C} \mathcal{A})_{\Omega}^{\mathrm{Arb}}$ models consistency and authenticity. More concretely, for dishonest senders $A_{i} \in \overline{\mathcal{S}^{H}},(\mathcal{C} \mathcal{A})_{\Omega}^{\mathrm{Arb}}$ includes the repository

$$
\left[\left\langle A_{i} \rightarrow \mathcal{V}\right\rangle_{\mathcal{V} \cup \overline{\mathcal{P}^{H}}}^{\overline{\mathcal{P}^{H}}}\right]_{A_{i} \in \overline{\mathcal{S}^{H}}, \mathcal{V} \subseteq \mathcal{R}}
$$

which captures consistency, since all honest receivers have access to the same messages. And for honest senders $A_{i} \in \mathcal{S}^{H},(\mathcal{C} \mathcal{A})_{\Omega}^{\text {Arb }}$ includes the repository

$$
\left[\overline{\mathcal{P}^{H}}\left\langle A_{i} \rightarrow \mathcal{V}\right\rangle_{\mathcal{V} \cup \overline{\mathcal{P}^{H}}}^{\left\{A_{i}\right\}}\right]_{A_{i} \in \mathcal{S}^{H}, \mathcal{V} \subseteq \mathcal{R}}
$$

which captures authenticity, since only $A_{i}$ can write. As before, a simulator sim is added at the interfaces of the dishonest parties, hence

$$
(\mathcal{C} \mathcal{A})_{\Omega}^{\text {Arb }}:=\left\{\operatorname{sim}^{\overline{\mathcal{P}^{H}}}\left[\begin{array}{c}
{\left[\left\langle A_{i} \rightarrow \mathcal{V}\right\rangle_{\mathcal{V} \cup \overline{\mathcal{P}^{H}}}^{\overline{\mathcal{P}^{H}}}\right]_{A_{i} \in \overline{\mathcal{S}^{H}}, \mathcal{V} \subseteq \mathcal{R}}} \\
{\left[\overline{\mathcal{P}^{H}}\left\langle A_{i} \rightarrow \mathcal{V}\right\rangle_{\mathcal{V} \cup \overline{\mathcal{P}^{H}}}^{\left\{A_{i}\right.}\right]_{A_{i} \in \mathcal{S}^{H}, \mathcal{V} \subseteq \mathcal{R}}}
\end{array}\right\}_{s i m \in \Omega}\right.
$$

Fig. 6 illustrates the ideal world systems from the $(\mathcal{C} \mathcal{A})_{\Omega}^{\text {Arb }}$ specification.

${ }^{21}$ This could have been modeled in Sect. 4 by adding a second Eve, but we omitted it for simplicity. 


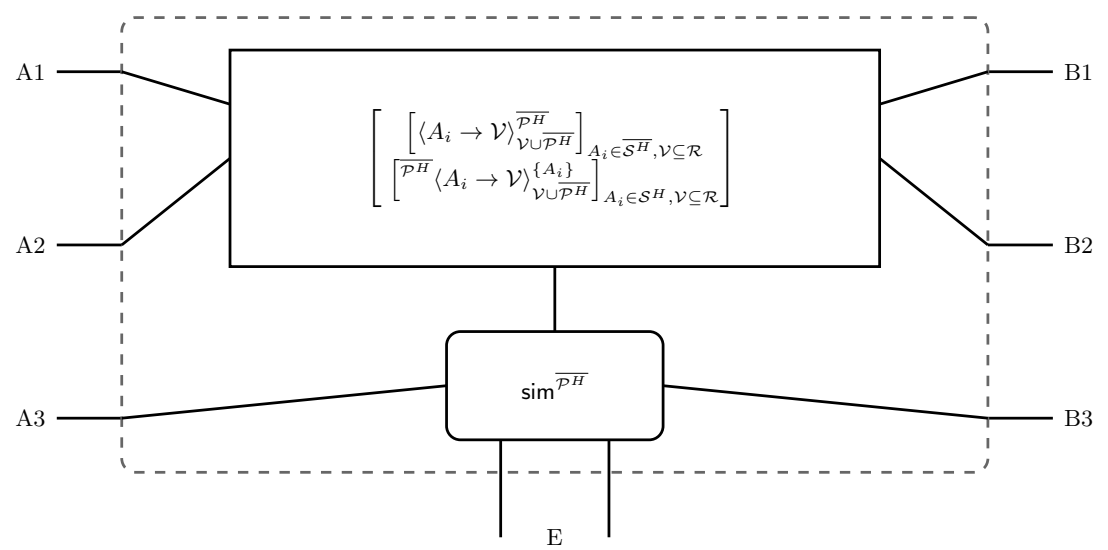

Fig. 6. Illustration of the ideal world system specified by Eq. (5.3) for the case where $\mathcal{S}=\{A 1, A 2, A 3\}, \mathcal{R}=\{B 1, B 2, B 3\}$, with $\mathcal{S}^{H}=\{A 1, A 2\}$ and $\mathcal{R}^{H}=\{B 1, B 2\}$.

Exclusiveness of Authenticity. To model exclusiveness of authenticity, for honest senders $A_{i} \in \mathcal{S}^{H}$, we define a resource containing a repository where $A_{i}$ and all dishonest parties (except Eve) can write and Eve can read, i.e.

$$
\left[\left\langle A_{i} \rightarrow \mathcal{V}\right\rangle_{\{E\}}^{\left\{A_{i}\right\} \cup \overline{\mathcal{S}^{H}} \cup \overline{\mathcal{R}^{H}}}\right]_{A_{i} \in \mathcal{S}^{H}, \mathcal{V} \subseteq \mathcal{R}} .
$$

This means that Eve does not know if the messages she sees are from Alice or another dishonest party - even those that are not designated verifiers can input messages.

In the arbitrary party setting, we also need to deal with the case of dishonest senders. Since we cannot exclude that by submitting forged signatures and seeing whether they are accepted, dishonest parties might learn something about the honest receivers' secret keys, we also include repositories where a dishonest party (Eve) can write and honest verifiers read ${ }^{22}$ namely

$$
\left[\left\langle A_{i} \rightarrow \mathcal{V}\right\rangle_{\mathcal{V}^{H}}^{\{E\}}\right]_{A_{i} \in \overline{\mathcal{S}^{H}}, \mathcal{V} \subseteq \mathcal{R}}
$$

Like in the previous section, we want to guarantee that the ability of dishonest parties to write in the repositories for honest senders is preserved, so the simulator

${ }^{22}$ Messages signed by a party with no knowledge of the signer's secret key will likely be recognized as forgeries, so we only need to consider the case where the sender is dishonest and the keys are shared. Furthermore, the distinguisher could in principle use any party's interface to submit these messages, but since it simplifies the presentation to only have the simulator at Eve's interface we only include Eve in the parties with write abilities. 
only covers Eve's interface ${ }^{23}$ We thus get a resource specification,

$$
\widehat{\mathcal{X}}_{\Omega}^{\mathrm{Arb}}:=\left\{\operatorname{sim}^{\{E\}}\left[\begin{array}{c}
{\left[\left\langle A_{i} \rightarrow \mathcal{V}\right\rangle_{\left.\mathcal{V}^{H}\right\}}^{\{E\}}\right]_{A_{i} \in \overline{\mathcal{S}^{H}}, \mathcal{V} \subseteq \mathcal{R}}} \\
{\left[\left\langle A_{i} \rightarrow \mathcal{V}\right\rangle_{\{E\}}^{\left\{A_{i}\right\} \cup \overline{\mathcal{S}}^{H} \cup \overline{\mathcal{R}}^{H}}\right]_{A_{i} \in \mathcal{S}^{H}, \mathcal{V} \subseteq \mathcal{R}}}
\end{array}\right] .\right.
$$

As previously, our ideal world consists of all resources that when the interfaces of the honest designated verifiers on repositories with honest senders are covered and when the dishonest parties (excluding Eve) collude to run a forging protocol $\pi$ result in a resource contained in $\widehat{\mathcal{X}}_{\Omega}^{\mathrm{Arb}}$, i.e. the ideal-world specification $\mathcal{X}_{\Omega, \pi}^{\mathrm{Arb}}$ is defined as

$$
\mathcal{X}_{\Omega, \pi}^{\mathrm{Arb}}:=\left\{\mathbf{V}: \pi^{\overline{\mathcal{S}^{H}} \cup \overline{\mathcal{R}^{H}}}\left(\perp_{\mathrm{Arb}}\right)^{\mathcal{R}^{H}} \mathbf{V} \in \widehat{\mathcal{X}}_{\Omega}^{\mathrm{Arb}}\right\},
$$

where $\perp_{\text {Arb }}$ is the converter specified in Algorithm 9 which does not allow the receiver to verify the authenticity of messages input into any repository $\left\langle A_{i} \rightarrow \mathcal{V}\right\rangle$ with an honest sender (i.e. for which $A_{i} \in \mathcal{S}^{H}$ ) ${ }^{24}$

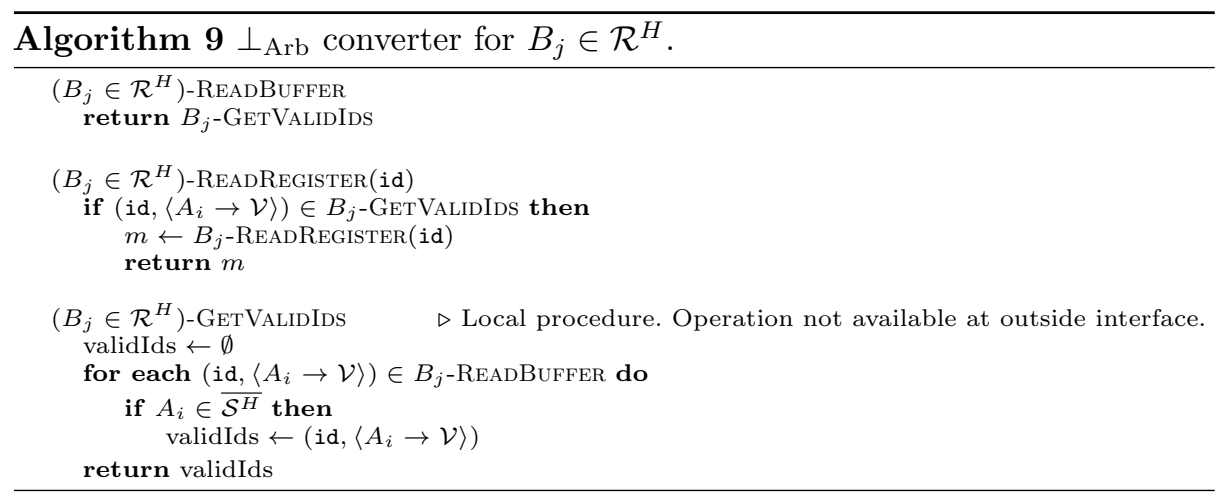

\subsection{Reduction to Game-Based Security}

We now compare our composable notions for arbitrary parties to the existing game-based security notions from the literature.

The first theorem in this section shows that that advantage in distinguishing the real world from the ideal world for authenticity and consistency is upper bounded by the advantage in winning the consistency, unforgeability and correctness games.

$\overline{23}$ Traditional composable security frameworks require the simulator to cover all dishonest interfaces making it impossible to model Eq. (5.4)

${ }^{24}$ Note that the ideal specification in Eq. (5.5) does not follow the ideal-functionalitysimulator paradigm, making it impossible to (directly) model the same thing in traditional composable frameworks. 
Theorem 4. Consider a setting where $\mathcal{R}^{H}, \overline{\mathcal{R}^{H}}, \mathcal{S}^{H}$ and $\overline{\mathcal{S}^{H}}$ are all non-empty. We find an explicit reduction system $\mathbf{C}^{\prime}$, an explicit simulator sim (see Algorithm 17) and explicit reduction systems $\mathbf{C}$ (see Algorithm 18), $\mathbf{C}_{\text {Cons }}$ (see Algorithm 19) and $\mathbf{C}_{\text {Unforg (see Algorithm 20) such that, for any } \Omega \supseteq\{\operatorname{sim}\}}$

$$
\mathcal{R} \subseteq\left((\mathcal{C} \mathcal{A})_{\Omega}^{\operatorname{Arb}}\right)^{A d v^{\text {Cons }}\left(\cdot \mathbf{C C}_{\text {Cons }}\right)+A d v^{\text {Unforg }}\left(\cdot \mathbf{C C}_{\text {Unforg }}\right)+A d v^{\text {Corr }}\left(\cdot \mathbf{C}^{\prime}\right)},
$$

where for any distinguisher $\mathbf{D}, A d v^{\text {Cons }}\left(\mathbf{D C C}_{\text {Cons }}\right), A d v^{\text {Unforg }}\left(\mathbf{D C C}_{\text {Unforg }}\right)$, and $A d v^{C o r r}\left(\mathbf{D C}^{\prime}\right)$ are, respectively, the advantages of $\mathbf{D}^{\prime}=\mathbf{D} \mathbf{C C}_{\text {Cons }}$ (the distinguisher resulting from composing $\mathbf{D}, \mathbf{C}$ and $\mathbf{C}_{\text {Cons }}$ ) in winning the Consistency game (see Definition 3), of $\mathbf{D}^{\prime \prime}=\mathbf{D} \mathbf{C C}_{\text {Unforg }}$ in winning the Unforgeability game (see Definition 4), and of $\mathbf{D}^{\prime \prime \prime}=\mathbf{D C}^{\prime}$ in winning the Correctness game (see Definition 2).

A proof of Theorem 4 is provided in Appendix A.4.

In the second theorem we show that the advantage in distinguishing the real world from the ideal world for the exclusiveness of authenticity is bounded by the advantage in winning the Off-The-Record and Consistency games.

Theorem 5. Consider a setting where $\mathcal{R}^{H}, \overline{\mathcal{R}^{H}}, \mathcal{S}^{H}$ and $\overline{\mathcal{S}^{H}}$ are all non-empty. For any signature forgery algorithm Forge suitable for the Off-The-Record security notion (see Definition 5), we find explicit reduction systems $\mathbf{C}$ (see Algorithm 22) and $\mathbf{C}^{\prime}$ (see Algorithm 23) and an explicit simulator sim (see Algorithm 21) such that for any $\Omega \supseteq\{\operatorname{sim}\}$ :

$$
\mathcal{R} \subseteq\left(\mathcal{X}_{\Omega, \pi \text { Forge }}^{\mathrm{Arb}}\right){ }^{\text {Adv }}{ }^{\text {OTR-Forge }(\cdot \mathbf{C})+A d v^{\text {Cons }}\left(\cdot \mathbf{C}^{\prime}\right)},
$$

where $\pi^{\text {Forge }}$ is the converter running the Forge algorithm (see Algorithm 10), and for any for any distinguisher $\mathbf{D}, A d v^{\text {OTR-Forge }}(\mathbf{D C})$ and $A d v^{C o n s}\left(\mathbf{D C}^{\prime}\right)$ are, respectively, the advantage of $\mathbf{D}^{\prime}=\mathbf{D C}$ (the distinguisher resulting from composing $\mathbf{D}$ and $\mathbf{C})$ in winning the Off-The-Record game with respect to forgery algorithm Forge(see Definition 5), and the advantage of $\mathbf{D}^{\prime \prime}=\mathbf{D C}^{\prime}$ in winning the Consistency game (see Definition 3).

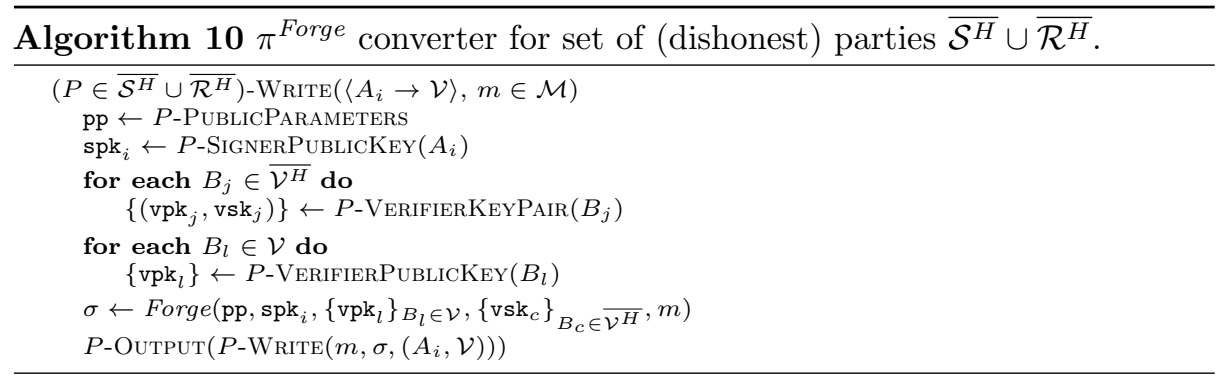

A proof of Theorem 5 is provided in Appendix A.5 
Asymptotic Composable Security of MDVS Analogously to Remark 2 for a security notion $\mathbf{X}, A d v^{\mathbf{X}}(\overrightarrow{\mathbf{A}}): \mathbb{N} \rightarrow \mathbb{R}$ denotes a function defined as $A d v^{\mathrm{X}}(\overrightarrow{\mathbf{A}})(k):=A d v^{\mathrm{X}}\left(\mathbf{A}_{k}\right)$. We say that a scheme satisfies $\mathbf{X}$ asymptotically if $A d v^{\mathrm{X}}(\overrightarrow{\mathbf{A}})$ is negligible on the security parameter $k$.

In the following, let $\Pi=\left(\right.$ Setup $, G_{S}, G_{V}$, Sign, Vfy $)$ be an MDVS scheme. The following corollaries, Corollary 1 and Corollary 2, follow from Theorem 4 and Theorem 5, respectively. These results state that any MDVS scheme $\Pi$ that is asymptotically secure - according to asymptotic versions of Definition 2 , Definition 3 Definition 4, and Definition 5 (see 11] for possible definitions) and which is used as specified in Sect. 5.1 asymptotically constructs, from a real world specification $\mathcal{R}$, the ideal world specification defined in Equation 5.2 (see Remark 2. Note that, since we are making asymptotic construction statements, $\Omega$ and $\Omega^{\prime}$ are both classes of efficient simulators (say non-uniform probabilistic polynomial time), and for any efficient family of distinguishers $\overrightarrow{\mathbf{D}}, \vec{\varepsilon}$ and $\vec{\varepsilon}^{\prime}$ are both negligible functions (on the security parameter).

Corollary 1. Consider a setting where $\mathcal{R}^{H}, \overline{\mathcal{R}^{H}}, \mathcal{S}^{H}$ and $\overline{\mathcal{S}^{H}}$ are all non-empty. If $\Pi$ is asymptotically Correct (see [21, Def. 2]), Consistent (see [21, Def. 3]) and Unforgeable (see [21, Def. 4]), then $\mathcal{R}$ asymptotically constructs $(\mathcal{C} \mathcal{A})^{\mathrm{Arb}}$.

Corollary 2. Consider a setting where $\mathcal{R}^{H}, \overline{\mathcal{R}^{H}}, \mathcal{S}^{H}$ and $\overline{\mathcal{S}^{H}}$ are all nonempty. If $\Pi$ is asymptotically Off-The-Record (see [21, Def. 5]) and Consistent (see [21, Def. 3]), then $\mathcal{R}$ asymptotically constructs $\mathcal{X}_{\pi^{\text {Forge }}}^{\text {Arb }}$, where $\pi^{\text {Forge }}$ is the converter defined in Algorithm 10, running an algorithm Forge with respect to which $\Pi$ is asymptotically Off-The-Record (i.e. no non-uniform probabilistic polynomial time adversary $\overrightarrow{\mathbf{A}}$ can win the Off-The-Record game of $\Pi$ with respect to algorithm Forge with non-negligible advantage).

\subsection{Separation from Existing Game-Based Security Notions}

The game-based security notion from 11 capturing the Off-The-Record security property of MDVS schemes - corresponding to Definition 5- is unnecessarily strong as for some MDVS schemes it allows the adversary to verify the validity of the challenge signatures, and thus allows it to trivially win the game. As hinted by our composable security notions, the main goal of the Off-The-Record security notion is capturing that a third party cannot tell whether a given signature is a valid one generated by the signer, or a forged one generated by dishonest receivers. The ability of a third party to generate signature replays - which might only be valid if the original signatures were already valid - does not violate any of the security properties that MDVS schemes intend to guarantee, and as such should not help in winning the corresponding security game. However, it does help in winning the Off-The-Record game from [11], meaning that this notion (i.e. the one from [11]) is unnecessarily strong.

Theorem 6. Let $\mathcal{P}=\left\{A_{1}, A_{2}, A_{3}, B_{1}, B_{2}, B_{3}, E\right\}$. Consider any MDVS scheme $\Pi$, and let $\varepsilon_{\Pi \Psi}$ and $\varepsilon_{\Pi[}$ denote the $\varepsilon$-balls (see Eq. (2.3)) given by, respectively, 
Theorem 4 and Theorem 5 for settings where $\mathcal{R}^{H}, \overline{\mathcal{R}^{H}}, \mathcal{S}^{H}$ and $\overline{\mathcal{S}^{H}}$ are all non-empty sets. Then there is a modified MDVS scheme $\Pi^{\prime}$ that is also secure as in each of these two theorems and for essentially the same $\varepsilon$-balls as $\Pi$, but such that for any suitable algorithm Forge for the Off-The-Record security notion(see Definition 5) there is an explicit and efficient adversary $\mathbf{A}$ such that

$$
A d v^{\Pi^{\prime}-O T R \text {-Forge }}(\mathbf{A}) \geq 1-\delta_{\text {corr }}-\delta_{\text {auth }},
$$

where $A d v^{\Pi^{\prime}-O T R \text {-Forge }}(\mathbf{A})$ denotes the advantage of $\mathbf{A}$ in winning the Off-TheRecord game for $\Pi^{\prime}$ with respect to the signature forgery algorithm Forge, $\delta_{\text {corr }}$ is the probability that a single honestly generated signature does not verify correctly and $\delta_{\text {auth }}$ is the probability that a single forged signature is considered valid by the signature verification algorithm.

A proof of Theorem 6 is provided in Appendix A.6

\section{Further Related Work}

In 13], Jakobsson, Sako, and Impagliazzo introduce DVS and MDVS schemes and give two property-based security notions for the single designated verifier case. Their weaker notion is intended to capture essentially the same as our weaker exclusiveness of authenticity notion - if all receivers are honest, Eve learns that Alice is the one sending messages - whereas their stronger notion is intended to capture our stronger notion - even if all receivers are honest, Eve cannot tell if Alice sent any message. Unfortunately, the signature unforgeability notion considered - equivalent to Existential Unforgeability under No-Message Attacks (EUF-NMA) - is known to be too weak to allow for authentic communication ${ }^{25}$ Furthermore, the security notion capturing the exclusiveness of authenticity which is implicitly considered for the case of multiple receivers is also too weak, and in particular is not sufficient to achieve neither of our composable notions. This is so since simulating signatures requires secret information from every designated verifier, and thus if at least one of the verifiers is honest, doing so is not feasible.

In [29], Steinfeld, Bull, Wang and Pieprzyk introduce Universal Designated Verifier Signatures, wherein a signer can generate publicly verifiable signatures which can then be transformed into designated verifier ones (possibly by a distinct party not possessing the secret signing key). Although the security notions capturing the exclusiveness of authenticity property introduced in that paper are weak - in that they only meet the weaker notion we introduce in this paper - the proposed schemes meet our stronger notion for this property (for the single receiver case). On the other hand, the unforgeability notion considered in the paper is too weak: it does not suffice to achieve even our weaker composable

${ }^{25}$ Existential Unforgeability under Chosen Message Attacks (EUF-CMA) - a security notion known to be strictly stronger than EUF-NMA - is necessary for authentic communication, see 3,7 . 
security notion. Unfortunately, numerous subsequent works have considered the same unforgeability notion \begin{tabular}{ll|l|l|l}
16 & 19 & 32 & 32
\end{tabular} .

In 15, Krawczyk and Rabin introduce Chameleon signature schemes, which work by first using a chameleon hash function to hash a message and then using a normal signature scheme to sign the resulting hash. Chameleon hash functions are public key schemes which are collision-resistant for anyone not possessing the secret key, but which allow for efficient collision finding given the secret key. The intended use of these schemes is to provide the same guarantees as DVS schemes: a designated receiver first generates its chameleon hash function, and sends the corresponding public key to the signer; the signer then sends a signature on the message under the hash function provided by the receiver, which it can verify. Since the receiver knows the secret key of the chameleon hash function it sent to the signer, no one other than the receiver gets convinced that the signer signed any particular message. However, these schemes do not allow to achieve the exclusiveness of authenticity that our stronger composable notion captures: anyone with the public keys of the signer and of the chameleon hash function can verify whether a certain signature is a valid one (for some message), which implies that no third-party can feasibly forge signatures that are indistinguishable from real ones (or otherwise the signature scheme used by the signer is not unforgeable). Moreover, they also do not achieve our weaker notion, as dishonest receivers can only forge signatures once the signer signed a message.

In 27, Rivest, Shamir and Tauman mention that two party ring signatures are DVS schemes. Indeed, one can obtain a DVS scheme meeting our weaker composable notion for the case of a single receiver $B$ by taking a ring signature scheme and using it to produce signatures for a ring composed by the signer $A$ and by the intended (designated) receiver of that message, $B{ }^{26}$ But notice that, similarly to the case of Chameleon signature schemes, public keys are enough to verify signatures, implying that the DVS schemes yielded by ring signatures can really only achieve our weaker security notion-where if both $A$ and $B$ are honest, $E$ learns $A$ is the signer. Furthermore, since any ring member can locally sign messages that are valid with respect to the entire ring, which is incompatible with the stronger authenticity requirement of MDVS schemes, ring signatures may only be used as DVS schemes for the case of a single receiver. Unfortunately, this went unnoticed in various prior works $9,16,18$, which gave constructions of MDVS schemes based on ring signature schemes.

One could think that perhaps, to achieve our stronger notion for exclusiveness of authenticity - where a third party is not convinced that the signer signed some message even when all the designated receivers (and the signer) are honestit suffices to guarantee that the validity of a signature can only be efficiently determined with the secret key given as input [28]. However, this is not the case. Consider for example, the case where the sender and the designated receivers share the signing key dsk of some (traditional) Digital Signature Scheme (DSS)

$\overline{26}$ As one might note, the resulting DVS scheme can only meet our weaker composable notion if the underlying ring signature scheme meets the stronger Anonymity against Attribution Attacks 4, Def. 4]. 
(with the corresponding verification key dvk being publicly known), and where the MDVS signature $\sigma_{m}$ for each message $m$ also includes a signature $\sigma_{m}{ }^{\prime}$ under dsk on $m$. Then, while to verify the validity of an MDVS signature $\sigma_{m}$ one may need the secret verification key for the MDVS scheme, by verifying the corresponding $\sigma_{m}{ }^{\prime}$ using dvk signature a third party already gets convinced, in the case where the sender and all the designated receivers are honest, that the really signer signed $m$. This same reasoning also explains why, in general, MAC schemes cannot be used per se as DVS schemes (in the stronger sense, captured by our stronger composable notion) for the two party case: it may not be feasible to simulate MAC schemes which look just like real ones.

\section{References}

1. Backes, M., Hofheinz, D.: How to break and repair a universally composable signature functionality. In: Zhang, K., Zheng, Y. (eds.) ISC 2004. LNCS, vol. 3225, pp. 61-72. Springer, Heidelberg (Sep 2004)

2. Backes, M., Pfitzmann, B., Waidner, M.: The reactive simulatability (RSIM) framework for asynchronous systems. Cryptology ePrint Archive, Report 2004/082 (2004), https://eprint.iacr.org/2004/082

3. Badertscher, C., Maurer, U., Tackmann, B.: On composable security for digital signatures. In: Abdalla, M., Dahab, R. (eds.) PKC 2018, Part I. LNCS, vol. 10769, pp. 494-523. Springer, Heidelberg (Mar 2018). https://doi.org/10.1007/978-3-31976578-5:17

4. Bender, A., Katz, J., Morselli, R.: Ring signatures: Stronger definitions, and constructions without random oracles. In: Halevi, S., Rabin, T. (eds.) TCC 2006. LNCS, vol. 3876, pp. 60-79. Springer, Heidelberg (Mar 2006). https://doi.org/10.1007/11681878*4

5. Canetti, R.: Universally composable security: A new paradigm for cryptographic protocols. In: 42nd FOCS. pp. 136-145. IEEE Computer Society Press (Oct 2001). https://doi.org/10.1109/SFCS.2001.959888

6. Canetti, R.: Universally composable signatures, certification and authentication. Cryptology ePrint Archive, Report 2003/239 (2003), https://eprint.iacr.org/2003/239

7. Canetti, R.: Universally composable signature, certification, and authentication. In: 17th IEEE Computer Security Foundations Workshop, (CSFW-17 2004), 28-30 June 2004, Pacific Grove, CA, USA. p. 219. IEEE Computer Society (2004). https://doi.org/10.1109/CSFW.2004.24, http://doi.ieeecomputersociety.org/10.1109/CSFW.2004.24

8. Canetti, R., Dodis, Y., Pass, R., Walfish, S.: Universally composable security with global setup. In: Vadhan, S.P. (ed.) TCC 2007. LNCS, vol. 4392, pp. 61-85. Springer, Heidelberg (Feb 2007). https://doi.org/10.1007/978-3-540-70936-74

9. Chow, S.S.M.: Multi-designated verifiers signatures revisited. Int. J. Netw. Secur. 7(3), 348-357 (2008), http://ijns.jalaxy.com.tw/contents/ijns-v7-n3/ijns-2008-v7n3-p348-357.pdf

10. Coretti, S., Maurer, U., Tackmann, B.: Constructing confidential channels from authenticated channels - public-key encryption revisited. In: Sako, K., Sarkar, P. (eds.) ASIACRYPT 2013, Part I. LNCS, vol. 8269, pp. 134-153. Springer, Heidelberg (Dec 2013). https://doi.org/10.1007/978-3-642-42033-78 
11. Damgård, I., Haagh, H., Mercer, R., Nitulescu, A., Orlandi, C., Yakoubov, S.: Stronger security and constructions of multi-designated verifier signatures. In: Pass, R., Pietrzak, K. (eds.) TCC 2020, Part II. LNCS, vol. 12551, pp. 229-260. Springer, Heidelberg (Nov 2020). https://doi.org/10.1007/978-3-030-64378-29

12. Hofheinz, D., Shoup, V.: GNUC: A new universal composability framework. Journal of Cryptology 28(3), 423-508 (Jul 2015). https://doi.org/10.1007/s00145-013-9160$\mathrm{y}$

13. Jakobsson, M., Sako, K., Impagliazzo, R.: Designated verifier proofs and their applications. In: Maurer, U.M. (ed.) EUROCRYPT'96. LNCS, vol. 1070, pp. 143 154. Springer, Heidelberg (May 1996). https://doi.org/10.1007/3-540-68339-9¹3

14. Jost, D., Maurer, U.: Overcoming impossibility results in composable security using interval-wise guarantees. In: Micciancio, D., Ristenpart, T. (eds.) CRYPTO 2020, Part I. LNCS, vol. 12170, pp. 33-62. Springer, Heidelberg (Aug 2020). https://doi.org/10.1007/978-3-030-56784-2*2

15. Krawczyk, H., Rabin, T.: Chameleon signatures. In: NDSS 2000. The Internet Society (Feb 2000)

16. Laguillaumie, F., Vergnaud, D.: Multi-designated verifiers signatures. In: López, J., Qing, S., Okamoto, E. (eds.) ICICS 04. LNCS, vol. 3269, pp. 495-507. Springer, Heidelberg (Oct 2004)

17. Laguillaumie, F., Vergnaud, D.: Designated verifier signatures: Anonymity and efficient construction from any bilinear map. In: Blundo, C., Cimato, S. (eds.) SCN 04. LNCS, vol. 3352, pp. 105-119. Springer, Heidelberg (Sep 2005). https://doi.org/10.1007/978-3-540-30598-9*8

18. Li, Y., Susilo, W., Mu, Y., Pei, D.: Designated verifier signature: Definition, framework and new constructions. In: Indulska, J., Ma, J., Yang, L.T., Ungerer, T., Cao, J. (eds.) Ubiquitous Intelligence and Computing, 4th International Conference, UIC 2007, Hong Kong, China, July 11-13, 2007, Proceedings. Lecture Notes in Computer Science, vol. 4611, pp. 1191-1200. Springer (2007). https://doi.org/10.1007/978-3540-73549-6_116, https://doi.org/10.1007/978-3-540-73549-6_116

19. Lipmaa, H., Wang, G., Bao, F.: Designated verifier signature schemes: Attacks, new security notions and a new construction. In: Caires, L., Italiano, G.F., Monteiro, L., Palamidessi, C., Yung, M. (eds.) ICALP 2005. LNCS, vol. 3580, pp. 459-471. Springer, Heidelberg (Jul 2005). https://doi.org/10.1007/11523468*38

20. Maurer, U.: Constructive cryptography - a new paradigm for security definitions and proofs. In: Proceedings of Theory of Security and Applications, TOSCA 2011. Lecture Notes in Computer Science, vol. 6993, pp. 33-56. Springer (2012). https://doi.org/10.1007/978-3-642-27375-9*3

21. Maurer, U., Portmann, C., Rito, G.: Giving an adversary guarantees (or: How to model designated verifier signatures in a composable framework). Cryptology ePrint Archive, Report 2021/1185 (2021), https://eprint.iacr.org/2021/1185

22. Maurer, U., Renner, R.: Abstract cryptography. In: Chazelle, B. (ed.) ICS 2011. pp. 1-21. Tsinghua University Press (Jan 2011)

23. Maurer, U., Renner, R.: From indifferentiability to constructive cryptography (and back). In: Hirt, M., Smith, A.D. (eds.) TCC 2016-B, Part I. LNCS, vol. 9985, pp. 3-24. Springer, Heidelberg (Oct / Nov 2016). https://doi.org/10.1007/978-3-66253641-4¹

24. Maurer, U.M.: Indistinguishability of random systems. In: Knudsen, L.R. (ed.) EUROCRYPT 2002. LNCS, vol. 2332, pp. 110-132. Springer, Heidelberg (Apr / May 2002). https://doi.org/10.1007/3-540-46035-7'8 
25. Maurer, U.M., Pietrzak, K., Renner, R.: Indistinguishability amplification. In: Menezes, A. (ed.) CRYPTO 2007. LNCS, vol. 4622, pp. 130-149. Springer, Heidelberg (Aug 2007). https://doi.org/10.1007/978-3-540-74143-5'8

26. Pfitzmann, B., Waidner, M.: A model for asynchronous reactive systems and its application to secure message transmission. In: 2001 IEEE Symposium on Security and Privacy. pp. 184-200. IEEE Computer Society Press (May 2001). https://doi.org/10.1109/SECPRI.2001.924298

27. Rivest, R.L., Shamir, A., Tauman, Y.: How to leak a secret. In: Boyd, C. (ed.) ASIACRYPT 2001. LNCS, vol. 2248, pp. 552-565. Springer, Heidelberg (Dec 2001). https://doi.org/10.1007/3-540-45682-1:32

28. Saeednia, S., Kremer, S., Markowitch, O.: An efficient strong designated verifier signature scheme. In: Lim, J.I., Lee, D.H. (eds.) ICISC 03. LNCS, vol. 2971, pp. 40-54. Springer, Heidelberg (Nov 2004)

29. Steinfeld, R., Bull, L., Wang, H., Pieprzyk, J.: Universal designated-verifier signatures. In: Laih, C.S. (ed.) ASIACRYPT 2003. LNCS, vol. 2894, pp. 523-542. Springer, Heidelberg (Nov / Dec 2003). https://doi.org/10.1007/978-3-540-40061533

30. Steinfeld, R., Wang, H., Pieprzyk, J.: Efficient extension of standard Schnorr/RSA signatures into universal designated-verifier signatures. In: Bao, F., Deng, R., Zhou, J. (eds.) PKC 2004. LNCS, vol. 2947, pp. 86-100. Springer, Heidelberg (Mar 2004). https://doi.org/10.1007/978-3-540-24632-9 7

31. Unruh, D., Müller-Quade, J.: Universally composable incoercibility. In: Rabin, T. (ed.) CRYPTO 2010. LNCS, vol. 6223, pp. 411-428. Springer, Heidelberg (Aug 2010). https://doi.org/10.1007/978-3-642-14623-7²2

32. Zhang, Y., Au, M.H., Yang, G., Susilo, W.: (strong) multi-designated verifiers signatures secure against rogue key attack. In: Xu, L., Bertino, E., Mu, Y. (eds.) Network and System Security - 6th International Conference, NSS 2012, Wuyishan, Fujian, China, November 21-23, 2012. Proceedings. Lecture Notes in Computer Science, vol. 7645, pp. 334-347. Springer (2012). https://doi.org/10.1007/978-3642-34601-9_25, https://doi.org/10.1007/978-3-642-34601-9_25

\section{Appendix}

\section{A Proofs}

\section{A.1 Proof of Theorem 1}

In order to prove Theorem 1 it suffices showing that simulator sim (specified in Algorithm 11 and reduction system $\mathbf{C}$ (specified in Algorithm 12) are such that, for any $\mathbf{D}$, the advantage of $\mathbf{D}$ in distinguishing the real world system from the ideal world system with the simulator attached is bounded by $A d v^{\text {Cons }}$ (DC): the advantage of DC in winning the Consistency game for the underlying MDVS scheme $\Pi$. By the definition of the $\varepsilon$-ball of a specification $E q .(2.3)$, Sect. 2.1 we then have that Theorem 1 follows from Lemma 1 below. 
Lemma 1. For $\mathcal{P}^{H}=\mathcal{R}^{H}$, reduction system $\mathbf{C}$ (see Algorithm 12) and simulator sim (see in Algorithm 11) are such that for any distinguisher $\mathbf{D}$

$$
\begin{aligned}
& \mid \Delta^{\mathbf{D}}\left(\operatorname{Rcv}^{\mathcal{R}^{H}}[\mathbf{K G A}, \mathbf{I N S}], \operatorname{sim}^{\overline{\mathcal{R}^{H}} \cup\{A, E\}}\left[\langle A \rightarrow \mathcal{R}\rangle_{\mathcal{R} \cup\{A, E\}}^{\left.\left.\overline{\mathcal{R}^{H}} \cup\{A, E\}\right]\right)} \mid\right.\right. \\
& \leq A d v^{\text {Cons }}(\mathbf{D C}) .
\end{aligned}
$$

Proof. Simulator sim, specified in Algorithm 11, initially generates the necessary keys and public parameters. Additionally, sim contains an internal repository SimRep to which each party $P \in \overline{\mathcal{R}^{H}} \cup\{A, E\}$ can both read and write (i.e. SimRep := SimRep $\frac{\overline{\mathcal{R}^{H}} \cup\{A, E\}}{\overline{\mathcal{R}^{H}} \cup\{A, E\}}$. More, the simulator also has a mapping GeneratedSignatures from register ids to signatures, a set AdvWrites of ids of registers generated by adversarial writes, and stores a verifier key-pair $\left(\mathrm{vpk}_{h}, \mathrm{vsk}_{h}\right)$ of an honest designated receiver $B_{h} \in \mathcal{R}^{H}$ (if there is one), which is used to decide whether each write into the repository is valid.

Let $\mathbf{R}$ denote the real world system, i.e.

$$
\mathbf{R}:=\operatorname{Rcv}^{\mathcal{R}^{H}}[\text { KGA, INS }],
$$

and $\mathbf{S}$ denote the ideal world system, i.e.

$$
\mathbf{S}:=\operatorname{sim}^{\overline{\mathcal{R}^{H}} \cup\{A, E\}}[\langle A \rightarrow \mathcal{R}\rangle] .
$$

We now show that systems $\mathbf{R}$ and $\mathbf{S}$ always behave indistinguishably, unless a distinguisher $\mathbf{D}$ performs a WRITE operation with input $\left(m^{*}, \sigma^{*}\right)$ at one of the adversarial interfaces $\overline{\mathcal{R}^{H}}, A, E$, returning an identifier id, such that there are two later READBUFFER invocations on the repository at interfaces $B_{h}$ and $B_{h^{\prime}}$ (where $B_{h}, B_{h^{\prime}} \in \mathcal{R}^{H}$ ) such that one of them returns a list containing id and the other returns a list not containing id. Let us denote this event by $\xi$. This means that the random systems $\mathbf{R}$ and $\mathbf{S}$ are indistinguishable up to event $\xi$ occurring, which implies that the distinguishing advantage between the two systems is bound by the probability of triggering event $\xi$ to occur (i.e. $\mathbf{R} \equiv{ }^{\xi} \mathbf{S}$ ).

We now prove $\mathbf{R} \equiv{ }^{\xi} \mathbf{S}$. Next, we will bound the probability of $\xi$ occurring by the success probability of an adversary in winning the consistency game of the underlying MDVS scheme. To argue about the indistinguishability of the random systems, note that their behavior is as follows:

Initialization Upon a call to this method by the distinguisher D, the KGA resource in the real world system and the simulator sim in the ideal world system both initialize, generating the public parameters and every signer and verifier key-pair necessary. The method has no output.

PublicParameters Upon a call to this method by the distinguisher $\mathbf{D}$ at the interface of any party $P \in \overline{\mathcal{R}^{H}} \cup\{A, E\}$, the public parameters pp for the underlying MDVS scheme generated by an invocation to Setup are returned to $\mathbf{D}$. For both systems, the public parameters and master secret key have the same distribution, and hence the public parameters have the same distribution. 


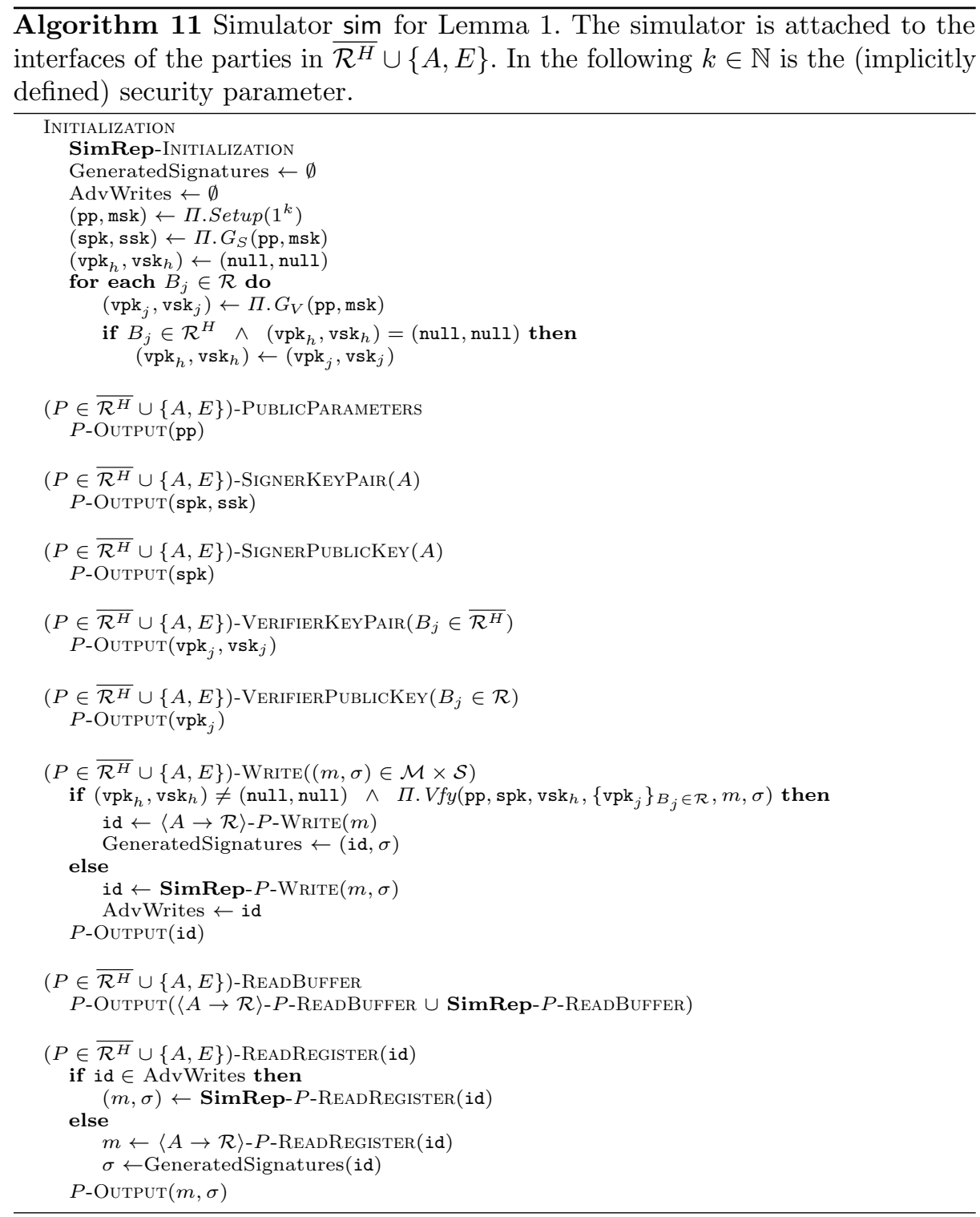




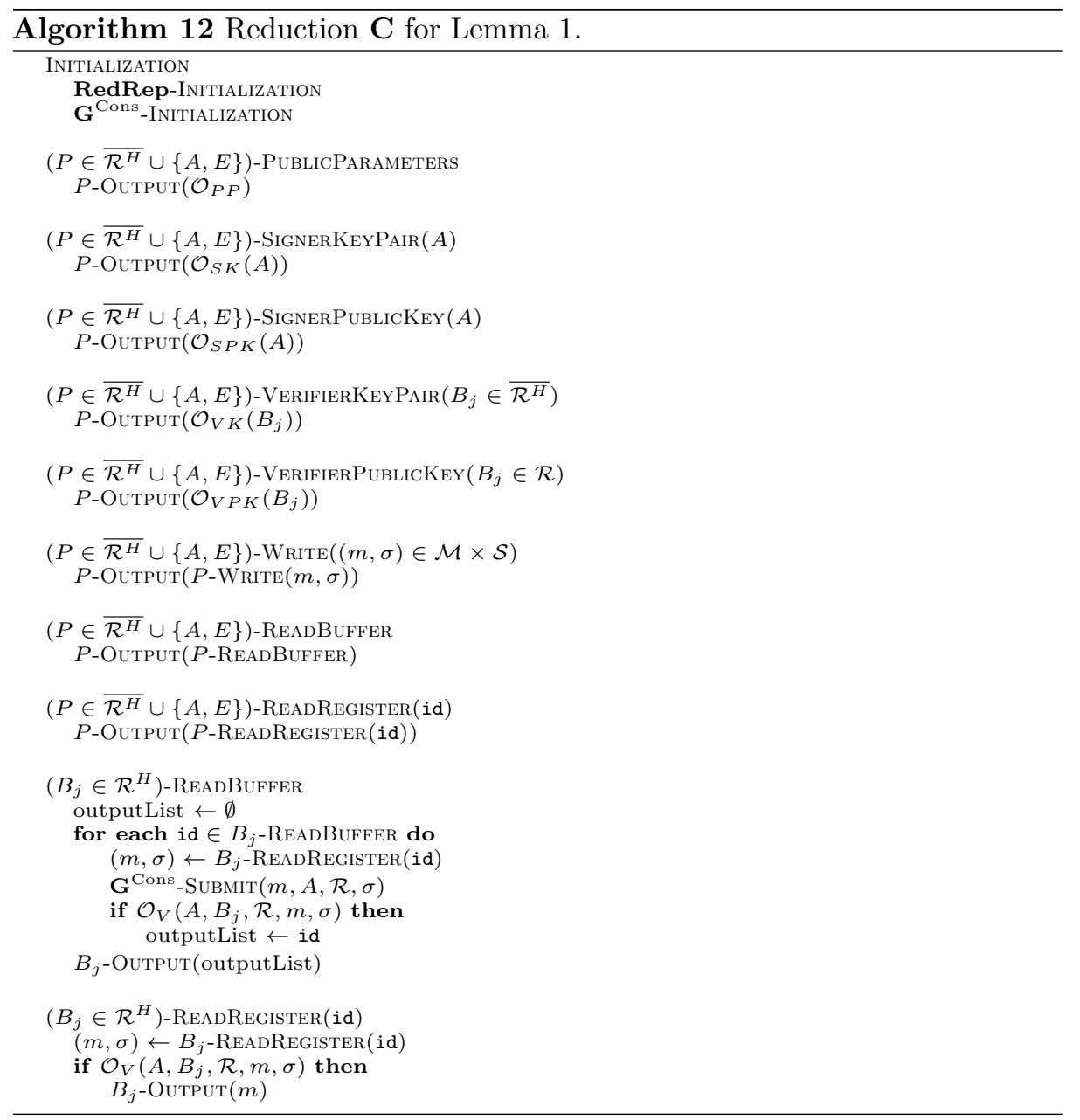


SignerKeyPair Upon a call to this method by the distinguisher at the interface of any party $P \in \overline{\mathcal{R}^{H}} \cup\{A, E\}$ with input $A$, the signer key-pair of $A$ is output back to $\mathbf{D}$ at the same interface. Again, in this case the output distribution is the same for both random systems.

SignerPublickey Upon an invocation of this method by $\mathbf{D}$ at the interface of any party $P \in \overline{\mathcal{R}^{H}} \cup\{A, E\}$, the public key of the signer passed as input to the method is returned. Following from the previous case, the public keys output in both systems have the same distribution.

VERIFIERKEYPAIR Upon a call to this method by $\mathbf{D}$ at the interface of a party $P \in \overline{\mathcal{R}^{H}} \cup\{A, E\}$ with input $B_{j} \in \overline{\mathcal{R}^{H}}$, the verifier key-pair of $B_{j}$ is output back to $\mathbf{D}$ (at the same interface $P$ ). The key-pairs output have the same distribution for both random systems.

VerifierPublicKey Upon a call by $\mathbf{D}$ at any party's interface $P \in \overline{\mathcal{R}^{H}} \cup$ $\{A, E\}$, the public key of the verifier passed as input to the method is returned to $\mathbf{D}$ again through $P$ 's interface. Again, as a consequence of the previous case, the public keys output by the random systems have the same distribution.

WRITE First, note that WRITE operations can only be issued at the interfaces of dishonest parties, as the only honest parties are the subset $\mathcal{R}^{H}$ of the designated receivers $\mathcal{R}$. Upon a call to this method by $\mathbf{D}$ at the interface of party $P \in \overline{\mathcal{R}^{H}} \cup\{A, E\}$ with input $(m, \sigma) \in \mathcal{M} \times \mathcal{S}$, the id of a new register is returned through $P$ 's interface back to $\mathbf{D}$. It is assumed that this id is unique among all repositories, and that the distribution of identifiers is the same for any repository. Thus, the output distribution is the same for both random systems upon any call to this method by $\mathbf{D}$. Regarding the internal effects of such invocations, in the ideal world system if $\sigma$ is a valid signature on $m$ with respect to sender $A$ and designated receivers $\mathcal{R}$, then $m$ is written into the ideal repository $(\langle A \rightarrow \mathcal{R}\rangle)$ and $\sigma$ is associated this write by being stored in the GeneratedSignatures mapping of the converter; if $\sigma$ is not a valid signature on $m$ with respect to sender $A$ and designated receivers $R$, then $(m, \sigma)$ is stored in the internal repository SimRep of the converter.

READBuffer for honest party Upon a call to this method by $\mathbf{D}$ through the interface of any party $P \in \mathcal{R}^{H}$, a list of register identifiers, where the list contains only the identifiers of registers which were authentically written into the ideal repository $\langle A \rightarrow \mathcal{R}\rangle$, is returned. First, note that assuming event $\xi$ does not occur, the number of register identifiers in the list returned back to $\mathbf{D}$ as the result of the call in the real world is the same regardless of which party $P \in \mathcal{R}^{H}$ 's interface $\mathbf{D}$ made the invocation through. Upon an invocation to the ideal world system, the list returned back to $\mathbf{D}$ is always the same, regardless of which party $P \in \mathcal{R}^{H}$ 's interface $\mathbf{D}$ made the invocation through. Second, given that the identifiers of the registers are identically distributed in the real and ideal world systems, the lists returned back to the distinguisher have the same distributions in both the real and ideal world systems.

READBufFer for dishonest party When $\mathbf{D}$ invokes this method through any party $P$ 's interface $\left(P \in \overline{\mathcal{R}^{H}} \cup\{A, E\}\right)$, a list of (all) register identifiers is 
returned. Since the distribution of register identifiers is the same for the repository as a whole in both the real and ideal worlds, then the distribution of the output which is sent back to $\mathbf{D}$ is the same.

READREGister for honest party When $\mathbf{D}$ invokes this method through any honest party $P$ 's interface $\left(P \in \mathcal{R}^{H}\right)$ giving as input the identifier of a register id, the message $m$ stored in the register with the given id is returned. The conditional distribution of the output messages $m$ given the input identifier id and the previous inputs and outputs to the system is the same in both the real and the ideal world systems under the assumption that the READBUfFER output is also indistinguishable, which in turn boils down to the assumption that $\xi$ does not occur.

READREGISTER for dishonest party Finally, when a dishonest party invokes this method giving as input some register id, a pair $(m, \sigma)$ is returned back to the caller. It is easy to see that the conditional distribution of the output is the same in both the real and the ideal world systems.

To prove the construction statement, we now bound the probability that event $\xi$ occurs. The reduction system $\mathbf{C}$ specified in Algorithm 12 has an internal repository RedRep, and emulates the real world $\mathbf{R}$ when given oracle access to the consistency game $\mathbf{G}^{\text {Cons }}$. During the emulation, $\mathbf{C}$ tries to win the game by submitting message-signature pairs input by $\mathbf{D}$ through dishonest parties interfaces as challenges to the game. It is easy to see from $\mathbf{C}$ 's specification (Algorithm 12 that the behaviors of $\mathbf{R}$ and of $\mathbf{C G}^{\text {Cons }}$ are perfectly indistinguishable, and the probability that $\xi$ occurs is upper bounded by the probability that the adversary $\mathbf{D C}$ wins $\mathbf{G}^{\text {Cons }}$ (as all the conditions for winning the game are met). Thus,

$$
\operatorname{Pr}^{\mathbf{D R}}[\xi]=\operatorname{Pr}^{\mathbf{D C G}}{ }^{\mathrm{Cons}}[\xi] \leq A d v^{\mathrm{Cons}}(\mathbf{D C}) .
$$

Finally, since event $\xi$ cannot occur in the ideal world by definition, $\operatorname{Pr}^{\mathbf{D S}}[\xi]=0$. Thus, for any distinguisher $\mathbf{D}$,

$$
\left|\Delta^{\mathbf{D}}(\mathbf{R}, \mathbf{S})\right| \leq \operatorname{Pr}^{\mathbf{D R}}[\xi] \leq A d v^{\text {Cons }}(\mathbf{D C}) .
$$

\section{A.2 Proof of Theorem 2}

Theorem 2 follows from Lemma 2 ahead, which we now prove.

Lemma 2. For $\mathcal{P}^{H}=\{A\} \cup \mathcal{R}^{H}$, there is an explicit reduction system $\mathbf{C}^{\prime}$, a reduction system $\mathbf{C}$ (see Algorithm 14) and a simulator $\operatorname{sim}$ (see Algorithm 13) are such that for any distinguisher $\mathbf{D}$ :

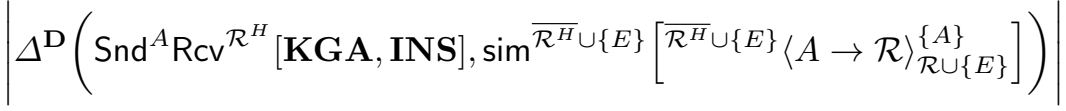

$$
\begin{aligned}
& \leq A d v^{\text {Unforg }}(\mathbf{D C})+A d v^{\operatorname{Corr}}\left(\mathbf{D C} \mathbf{C}^{\prime}\right)
\end{aligned}
$$




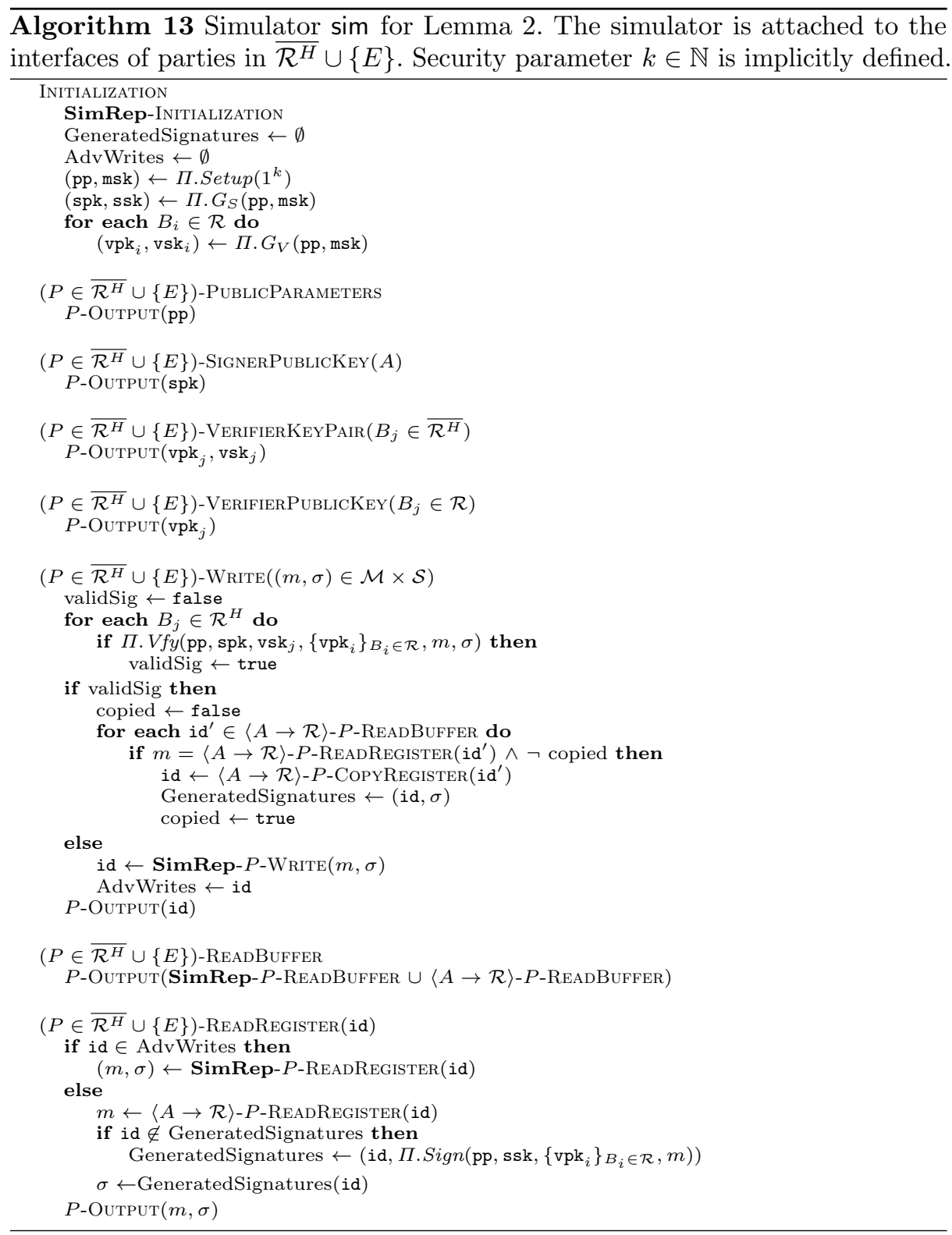




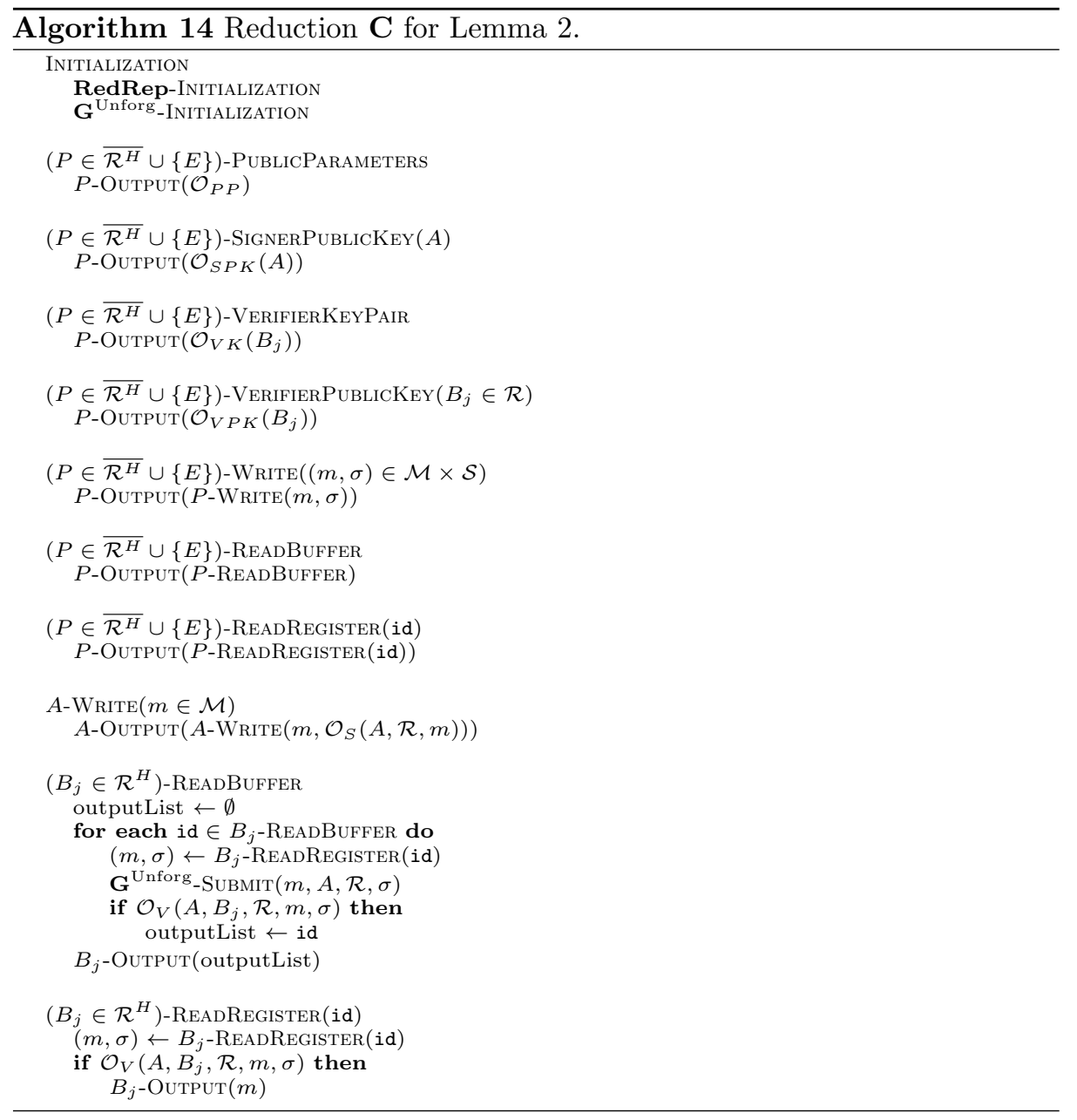


Proof. Simulator sim, specified in Algorithm 13, has an internal repository SimRep to which each party $P \in \overline{\mathcal{R}^{H}} \cup\{E\}$ can both read and write (i.e. SimRep := SimRep $\frac{\overline{\mathcal{R}^{H}} \cup\{E\}}{\overline{\mathcal{R}^{H}} \cup\{E\}}$, stores a mapping GeneratedSignatures from register ids to signatures and also stores a set AdvWrites of ids of registers generated by adversarial writes.

Let $\mathbf{R}$ denote the real world system, i.e.

$$
\mathbf{R}:=\mathrm{Snd}^{A} \operatorname{Rcv}^{\mathcal{R}^{H}}[\mathbf{K G A}, \mathbf{I N S}],
$$

and $\mathbf{S}$ denote the ideal world system, i.e.

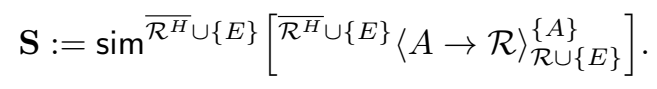

Next we will show that systems $\mathbf{R}$ and $\mathbf{S}$ always behave indistinguishably, unless a distinguisher $\mathbf{D}$

1. performs a WRITE operation with input $\left(m^{*}, \sigma^{*}\right)$ at one of the adversarial interfaces $\overline{\mathcal{R}^{H}}, E$ returning an identifier id such that (a) $\mathbf{D}$ did not previously issue a WRITE operation at the $A$ interface with input $m^{*}$, and (b) there is a later READBUFFER invocation at the interface of some (honest) receiver $B_{j} \in \mathcal{R}^{H}$ in which the register's identifier id is returned;

2. performs a WRITE operation with input $m^{*}$ at interface $A$ returning an identifier id such that there is a later READBUFFER invocation on the repository at the interface of some (honest receiver) $B_{j} \in \mathcal{R}^{H}$ and id is not in the list returned by the invocation. ${ }^{27}$

Let us denote the first event by $\xi_{1}$, the second by $\xi_{2}$, and the event that at least one of $\xi_{1}$ or $\xi_{2}$ occurs by $\xi$. This means that the random systems $\mathbf{R}$ and $\mathbf{S}$ are indistinguishable up to event $\xi$ occurring, which implies that the distinguishing advantage between the two systems is bounded by the probability of triggering event $\xi$, i.e. $\mathbf{R} \equiv{ }^{\xi} \mathbf{S}$. Noting that $\operatorname{Pr}^{\mathbf{D S}}\left[\xi_{1}\right]=\operatorname{Pr}^{\mathbf{D S}}\left[\xi_{2}\right]=0$, it follows $\operatorname{Pr}^{\mathbf{D S}}[\xi]=0$.

We now show that $\mathbf{R}$ and $\mathbf{S}$ are conditionally indistinguishable up to event $\xi$ occurring, meaning that if $\xi$ does not occur, then the systems are perfectly indistinguishable. Next, we will prove that the probability of $\xi$ occurring is bounded by the sum of the success probability of an adversary in winning the unforgeability game together with the probability that a fresh signature does not verify correctly. To argue about the indistinguishability of the real and ideal world random systems, note that their behavior is as follows:

Initialization Upon a call to this method by the distinguisher D, the KGA resource in the real world system and the simulator sim in the ideal world system both initialize, generating the public parameters and every signer and verifier key-pair necessary. The method has no output.

$\overline{27}$ Note that this event can only occur when $\mathbf{D}$ is interacting with the real world system R. 
PublicParameters Upon a call to this method by the distinguisher $\mathbf{D}$ at the interface of any party $P \in \overline{\mathcal{R}^{H}} \cup\{E\}$, the public parameters pp for the underlying MDVS scheme generated by an invocation to Setup are returned to $\mathbf{D}$. For both systems, the public parameters and master secret key have the same distribution, and hence an invocation of this procedure has the same behavior for both the real and the ideal world (with the simulator attached).

SignerPublickey Upon an invocation of this method by $\mathbf{D}$ at the interface of any party $P \in \overline{\mathcal{R}^{H}} \cup\{E\}$, the public key of the signer passed as input to the method is returned. Again, in this case the output distribution is the same for both random systems, as the signer's key-pair is honestly generated in both cases. Thus, the public keys output in both systems have the same distribution.

VERIFIERKEYPAIR Upon a call to this method by $\mathbf{D}$ at the interface of a dishonest party $P \in \overline{\mathcal{R}^{H}} \cup\{E\}$ with input $B_{j} \in \overline{\mathcal{R}^{H}}$, the verifier key-pair of $B_{j}$ is output back to $\mathbf{D}$ (at the same interface $P$ ). The key-pairs output have the same distribution for both random systems.

VerifierPublicKey Upon a call by $\mathbf{D}$ at any party's interface $P \in \overline{\mathcal{R}^{H}} \cup\{E\}$, the public key of the verifier passed as input to the method is returned to $\mathbf{D}$ again through $P$ 's interface. Again, as a consequence of the previous case, the verifier public keys output by the random systems have the same distribution.

Write When $\mathbf{D}$ issues a Write operation at the interface of the sender $A$, then the id of a new register is output back to $\mathbf{D}$ at the same interface. If $\mathbf{D}$ issues a WRITE operation at the interface of a dishonest party $P \in \overline{\mathcal{R}^{H}} \cup\{E\}$ with input $(m, \sigma) \in \mathcal{M} \times \mathcal{S}$, the id of a new register is returned through $P$ 's interface back to $\mathbf{D}$. It is assumed that this id is unique among all repositories, and that the distribution of identifiers is the same for any repository. Thus, the output distribution is the same for both random systems upon any call to this method by $\mathbf{D}$. Regarding the internal effects of such invocations, in the ideal world system if $\sigma$ is a valid signature on $m$ with respect to sender $A$, set of designated receivers $\mathcal{R}$ and any verifier secret key vsk ${ }_{j}$ of an honest receiver $B_{j} \in \mathcal{R}^{H}$, and in addition there is a register in the ideal world repository containing message $m^{\prime}=m$, then the register is copied and $\sigma$ is associated with this new (copied) register's id. If $\sigma$ is not a valid signature, then a new register is created at the internal (insecure) repository of the simulator. Finally, in the real world, a new register is created in the insecure repository.

ReAdBuffer for honest party Upon a call to this method by $\mathbf{D}$ through the interface of any party $P \in \mathcal{R}^{H}$, a list of register identifiers containing only the identifiers of registers which were authentically written into the repository is returned. First, note that assuming event $\xi$ does not occur, the number of register identifiers in the list returned back to $\mathbf{D}$ as the result of the call in the real world is the same regardless of which party $P \in \mathcal{R}^{H}$ 's interface $\mathbf{D}$ made the invocation through. Upon an invocation to the ideal world system, the list returned back to $\mathbf{D}$ is always the same, regardless of which party $P \in \mathcal{R}^{H}$ 's interface $\mathbf{D}$ made the invocation through. Second, given that the identifiers of the registers are identically distributed in the real and ideal 
world systems, the lists returned back to the distinguisher have the same distributions in both the real and ideal world systems.

READBUfFER for dishonest party When $\mathbf{D}$ invokes this method through any party $P \in \overline{\mathcal{R}^{H}} \cup\{E\}$ 's interface, a list of (all) register identifiers is returned. Since the distribution of register identifiers is the same for the repository as a whole in both the real and ideal worlds, then the distribution of the output which is sent back to $\mathbf{D}$ is the same.

READREGISTER for honest party When $\mathbf{D}$ invokes this method through any honest party $P \in \mathcal{R}^{H}$ 's interface giving as input the identifier of a register $i d$, the message $m$ stored in the register with the given id is returned. The conditional distribution of the output messages $m$ given the input identifier id and the previous inputs and outputs to the system is the same in both the real and the ideal world systems under the assumption that the READBUfFER output is also indistinguishable, which in turn boils down to the assumption that $\xi$ does not occur.

READREGISTER for dishonest party Finally, when a dishonest party invokes this method giving as input some register id, a pair $(m, \sigma)$ is returned back to the caller. It is easy to see that the conditional distribution of the output is the same in both the real and the ideal world systems.

To prove the construction statement, we now bound the probability that event $\xi$ occurs. By definition of $\xi$, it suffices to bound the probability that at least one of $\xi_{1}$ or $\xi_{2}$ occurs.

$\xi_{1}$ occurs Reduction system $\mathbf{C}$ (specified in Algorithm 14 satisfies $\mathbf{R} \equiv \mathbf{C G}^{\text {Unforg }}$ and is such that the probability for $\xi_{1}$ to occur is upper bounded by the probability that DC wins the unforgeability game for the underlying MDVS scheme, i.e.

$$
\operatorname{Pr}^{\mathbf{D R}}\left[\xi_{1}\right]=\operatorname{Pr}^{\mathbf{D C G}}{ }^{\text {Unforg }}\left[\xi_{1}\right] \leq A d v^{\text {Unforg }}(\mathbf{D C}) ;
$$

$\xi_{2}$ occurs The probability that $\xi_{2}$ occurs is bounded by the probability that the underlying MDVS scheme does not work correctly. It is easy to see that there is an explicit reduction system $\mathbf{C}^{\prime}$ satisfying $\mathbf{R} \equiv \mathbf{C}^{\prime} \mathbf{G}^{\text {Corr }}$ such that for any distinguisher $\mathbf{D}$ the probability that $\xi_{2}$ occurs is upper bounded by the probability that $\mathbf{D} \mathbf{C}^{\prime}$ wins the correctness game, i.e.

$$
\operatorname{Pr}^{\mathbf{D R}}\left[\xi_{2}\right]=\operatorname{Pr}^{\mathbf{D C}^{\prime} \mathbf{G}^{\mathrm{Corr}}}\left[\xi_{2}\right] \leq A d v^{\mathrm{Corr}}\left(\mathbf{D C}^{\prime}\right) .
$$

The reduction system $\mathbf{C}$ (specified in Algorithm 14) has an internal repository RedRep which it uses to store message-signature pairs and a set HonestWrites which it uses to keep track of all messages written into $A$ 's interface. During the emulation $\mathbf{C}$ tries to win the game by submitting message-signature pairs input by $\mathbf{D}$ through dishonest parties interfaces as challenges to the game. It is easy to see that the behaviors of $\mathbf{R}$ and of $\mathbf{C G}^{\text {Unforg }}$ are indeed perfectly indistinguishable, and that the probability that $\xi_{1}$ occurs is upper bounded by 
the probability that the $\mathbf{D C}$ wins the game, as all the required winning conditions are met. Since, as already mentioned, $\operatorname{Pr}^{\mathbf{D S}}[\xi]=0$, it follows

$$
\begin{aligned}
\left|\Delta^{\mathbf{D}}(\mathbf{R}, \mathbf{S})\right| & \leq \operatorname{Pr}^{\mathbf{D R}}[\xi] \\
& \leq \operatorname{Pr}^{\mathbf{D R}}\left[\xi_{1}\right]+\operatorname{Pr}^{\mathbf{D R}}\left[\xi_{2}\right] \\
& \leq A d v^{\text {Unforg }}(\mathbf{D C})+A d v^{\text {Corr }}\left(\mathbf{D C}^{\prime}\right) .
\end{aligned}
$$

\section{A.3 Proof of Theorem 3}

To prove Theorem 3, we prove the following result.

Lemma 3. For $\mathcal{P}^{H}=\{A\} \cup \mathcal{R}^{H}$ and for any signature forgery algorithm Forge suitable for the Off-The-Record security notion (see Definition 5), there is a protocol $\pi^{\text {Forge }}$ (see Algorithm 6) such that reduction system $\mathbf{C}$ (see Algorithm 16) and simulator sim (see Algorithm 15) satisfy, for any distinguisher $\mathbf{D}$ :

$$
\begin{aligned}
& \left|\Delta^{\mathbf{D}}\left(\perp^{\mathcal{R}^{H}}\left(\pi^{\text {Forge }}\right)^{\overline{\mathcal{R}^{H}}}\left(\operatorname{Snd}^{A} \operatorname{Rcv}^{\mathcal{R}^{H}}[\mathbf{K G A}, \mathbf{I N S}]\right), \operatorname{sim}^{\{E\}}\left[\langle A \rightarrow \mathcal{R}\rangle_{\{E\}}^{\overline{\mathcal{R}^{H}} \cup\{A\}}\right]\right)\right| \\
& =A d v^{\text {OTR-Forge }}(\mathbf{D C}) .
\end{aligned}
$$

Proof. As specified in Algorithm 6, when a party $B_{j} \in \overline{\mathcal{R}^{H}}$ invokes method WRITE to $\pi^{\text {Forge }}$ giving as input a message $m \in \mathcal{M}, \pi^{\text {Forge }}$ first fetches the public parameters, the public key of the signer and of each of the designated verifiers, and the secret keys of each of the dishonest verifiers, and then uses these to simulate a signature $\sigma$ on $m$. Finally, the converter writes the pair $(m, \sigma)$ into the insecure repository.

Simulator sim (specified in Algorithm 15) initially generates the necessary keys and public parameters. Additionally, sim contains an internal repository SimRep $:=\operatorname{SimRep}_{\{E\}}^{\{E\}}$ to which $E$ can both read and write. The simulator also stores a mapping GeneratedSignatures from register ids to signatures, and a set AdvWrites of ids of registers generated by adversarial writes.

Let $\mathbf{R}$ denote the real world system, i.e.

$$
\mathbf{R}:=\perp^{\mathcal{R}^{H}}\left(\pi^{\text {Forge }}\right)^{\overline{\mathcal{R}^{H}}}\left(\text { Snd }^{A} \operatorname{Rcv}^{\mathcal{R}^{H}}[\text { KGA }, \text { INS }]\right),
$$

and $\mathbf{S}$ denote the ideal world system, i.e.

$$
\mathbf{S}:=\operatorname{sim}^{\{E\}}\left[\langle A \rightarrow \mathcal{R}\rangle_{\{E\}}^{\overline{\mathcal{R}^{H}} \cup\{A\}}\right] .
$$

Reduction system $\mathbf{C}$, specified in Algorithm 16, has an internal repository RedRep that it uses to store message signature pairs. The reduction $\mathbf{C}$ essentially relies on the oracles provided by the game systems to emulate the KGA resource and uses the $\mathcal{O}_{\text {ChallengeSign }}$ oracle to get signatures on messages 


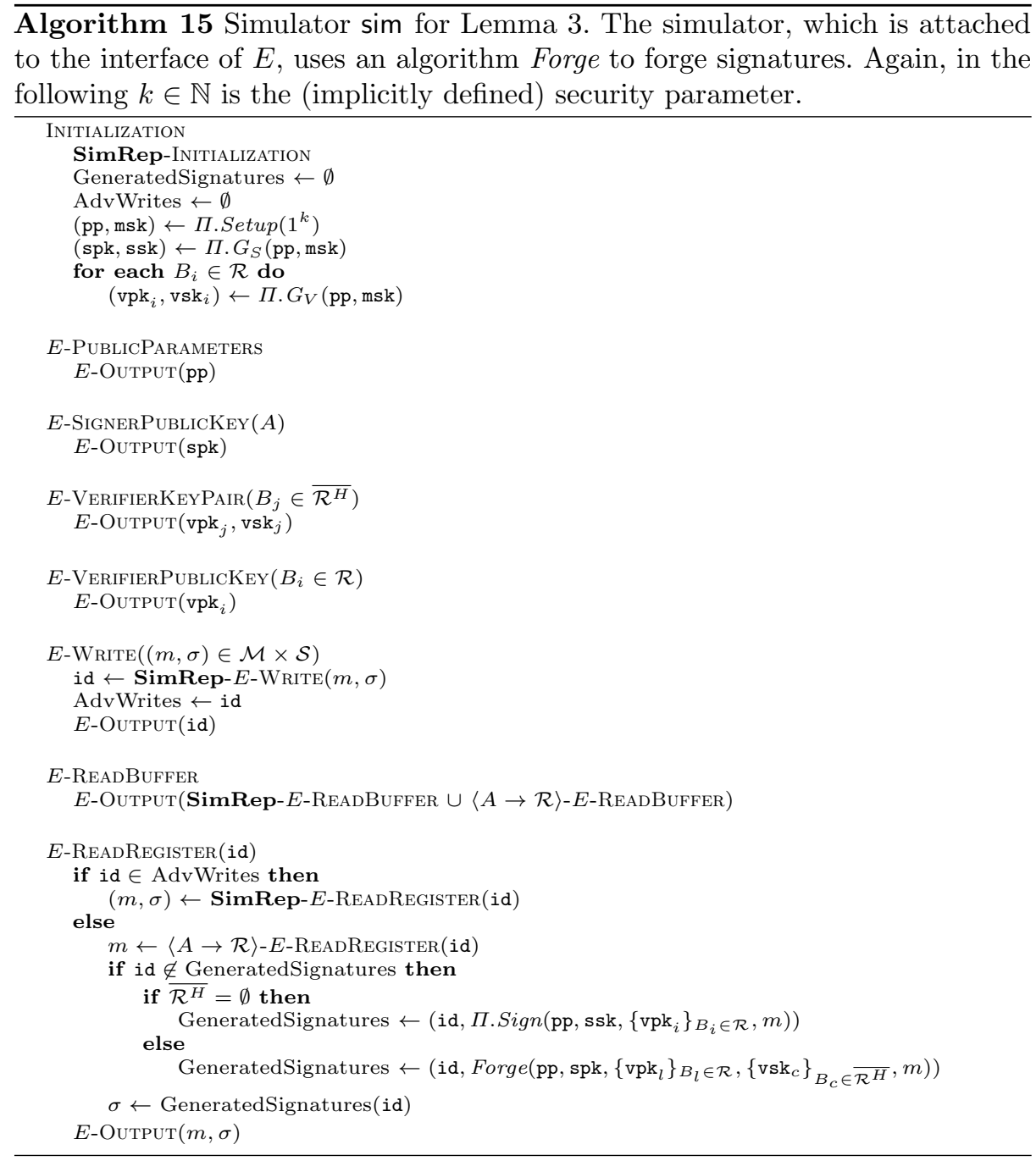




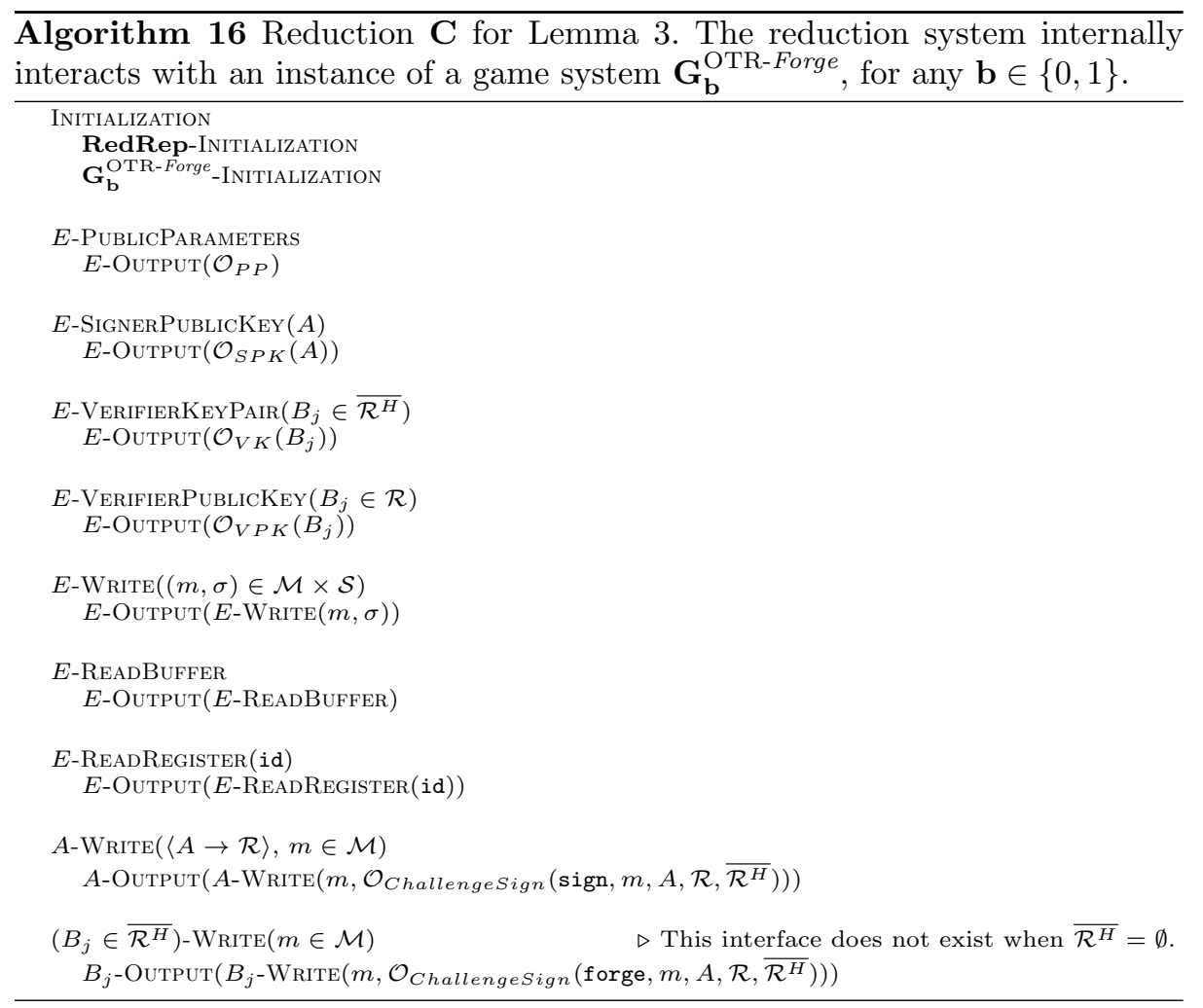


that are inserted at the interfaces of $A$ and of any dishonest receiver $\overline{\mathcal{R}^{H}}$. More concretely, if a message is input at $A, \mathbf{C}$ queries $\mathcal{O}_{\text {ChallengeSign }}$ with sign, as the message was input by the honest party $A$; if a message is input at the interface of party in $\overline{\mathcal{R}^{H}}$, then the reduction queries $\mathcal{O}_{\text {ChallengeSign }}$ with forge, as the message was input by a dishonest party in $\overline{\mathcal{R}^{H}}$. It is easy to see that, regardless of whether $\overline{\mathcal{R}^{H}}=\emptyset, \mathbf{C}$ perfectly emulates the real world $\mathbf{R}$ when connected to the game system $\mathbf{G}_{\mathbf{0}}^{\text {OTR-Forge }}$ (i.e. $\mathbf{R} \equiv \mathbf{C G}_{\mathbf{0}}^{\text {OTR-Forge }}$ ) and the ideal world $\mathbf{S}$ when connected to $\mathbf{G}_{\mathbf{1}}^{\text {OTR-Forge }}$ (i.e. $\mathbf{S} \equiv \mathbf{C G}_{\mathbf{1}}^{\text {OTR-Forge }}$ ). Thus, for any distinguisher $\mathbf{D}$,

$$
\begin{aligned}
\left|\Delta^{\mathbf{D}}(\mathbf{R}, \mathbf{S})\right|= & |\operatorname{Pr}[\mathbf{D S}=1]-\operatorname{Pr}[\mathbf{D R}=1]| \\
= & |\operatorname{Pr}[\mathbf{D S}=1]+\operatorname{Pr}[\mathbf{D R}=0]-1| \\
= & \mid \operatorname{Pr}\left[\mathbf{D C} \mathbf{G}_{\mathbf{1}}^{\text {OTR-Forge }}=\text { win }\right] \\
& \quad+\operatorname{Pr}\left[\mathbf{D C} \mathbf{G}_{\mathbf{0}}^{\text {OTR-Forge }}=\text { win }\right]-1 \mid \\
= & \text { Adv }{ }^{\text {OTR-Forge }}(\mathbf{D C}) .
\end{aligned}
$$

\section{A.4 Proof of Theorem 4}

Theorem 4 follows from Lemma 4, which we now prove.

Lemma 4. Consider a setting where $\mathcal{R}^{H}, \overline{\mathcal{R}^{H}}, \mathcal{S}^{H}$ and $\overline{\mathcal{S}^{H}}$ are all non-empty. There is an explicit reduction system $\mathbf{C}^{\prime}$, a simulator $\operatorname{sim}$ (defined in Algorithm 17) and reduction systems $\mathbf{C}$ (see Algorithm 18), $\mathbf{C}_{\text {Cons }}$ (see Algorithm 19) and $\mathbf{C}_{\text {Unforg }}$ (see Algorithm 20) satisfying, for any distinguisher $\mathbf{D}$

$$
\begin{aligned}
& \Delta^{\mathbf{D}}\left(\mathrm{Snd}^{\mathrm{Arb}^{\mathcal{S}^{H}}} \mathrm{Rcv}^{\mathrm{Arb}^{\mathcal{R}^{H}}}[\mathbf{K G A}, \mathbf{I N S}],\right.
\end{aligned}
$$

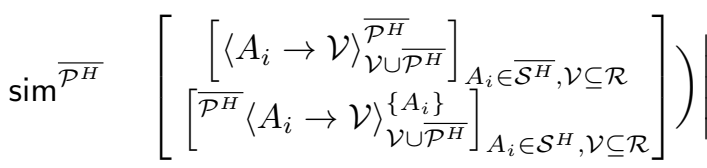

$$
\begin{aligned}
& \leq A d v^{\text {Cons }}\left(\mathbf{D C} \mathbf{C}_{\text {Cons }}\right)+A d v^{\text {Unforg }}\left(\mathbf{D C C}_{U n f o r g}\right)+A d v^{C o r r}\left(\mathbf{D C}^{\prime}\right) \text {. }
\end{aligned}
$$

Proof. Simulator sim, specified in Algorithm 17, initially generates the necessary keys and public parameters. Additionally, sim contains an internal repository SimRep to which each party $P \in \overline{\mathcal{P}^{H}}$ can read and write (i.e. SimRep $:=$ $\operatorname{SimRep} \frac{\overline{\mathcal{P}^{H}}}{\mathcal{P}^{H}}$. More, the simulator also has a mapping GeneratedSignatures from register ids to quadruples message-signature-sender-receivers and a set AdvWrites of ids of registers generated by adversarial writes.

Let $\mathbf{R}$ denote the real world system

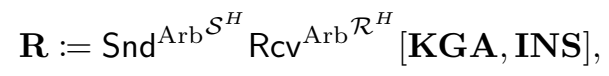




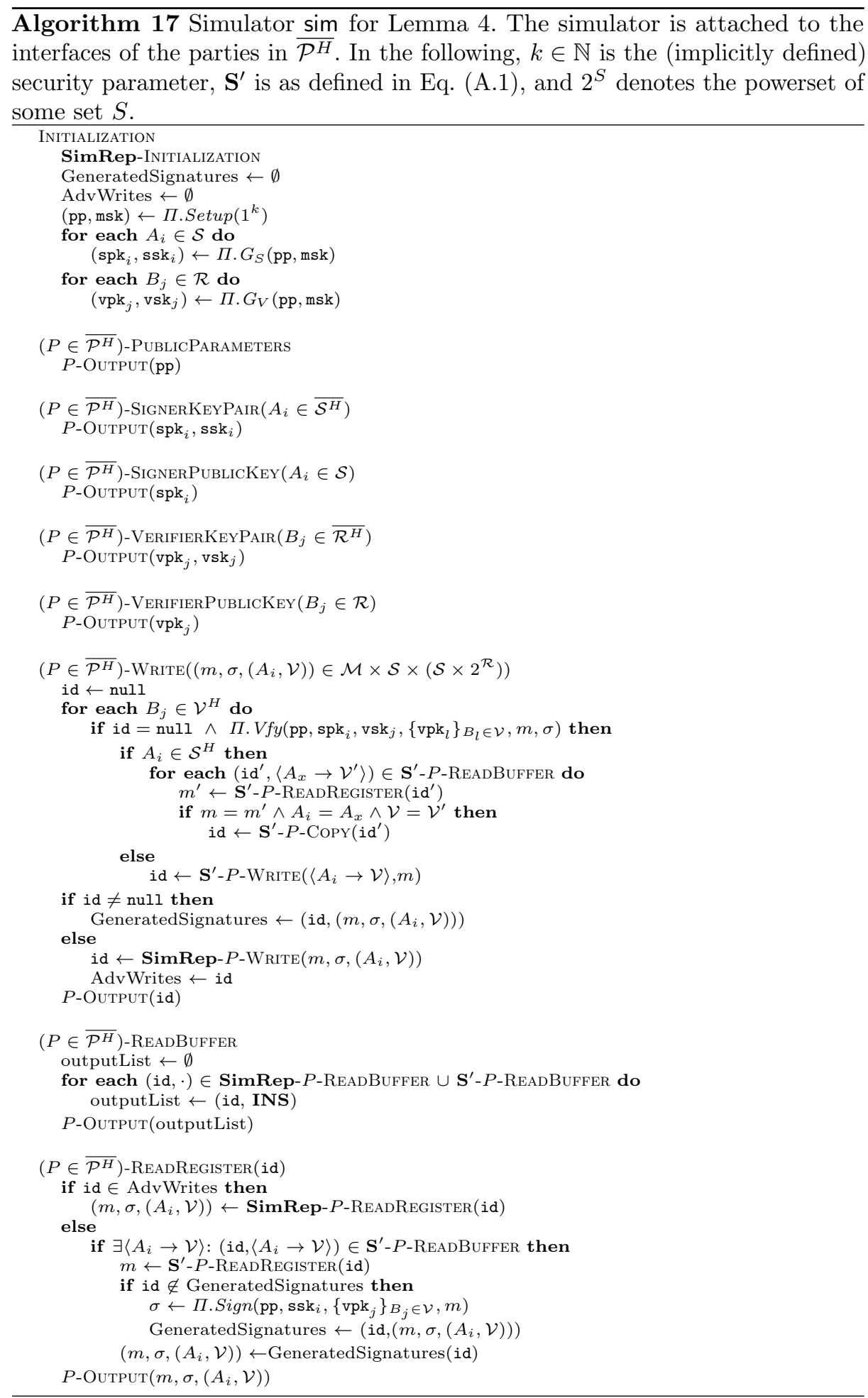




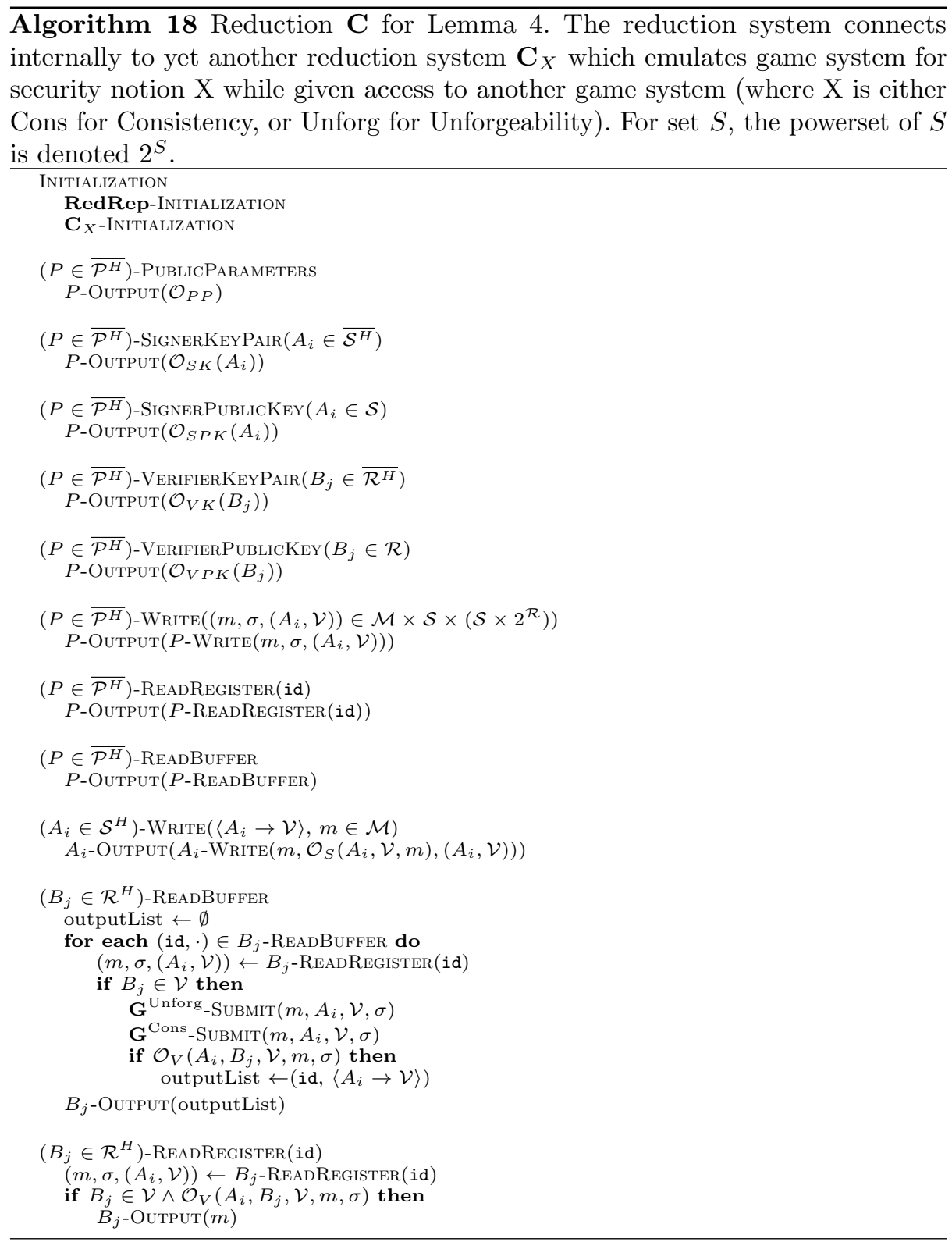



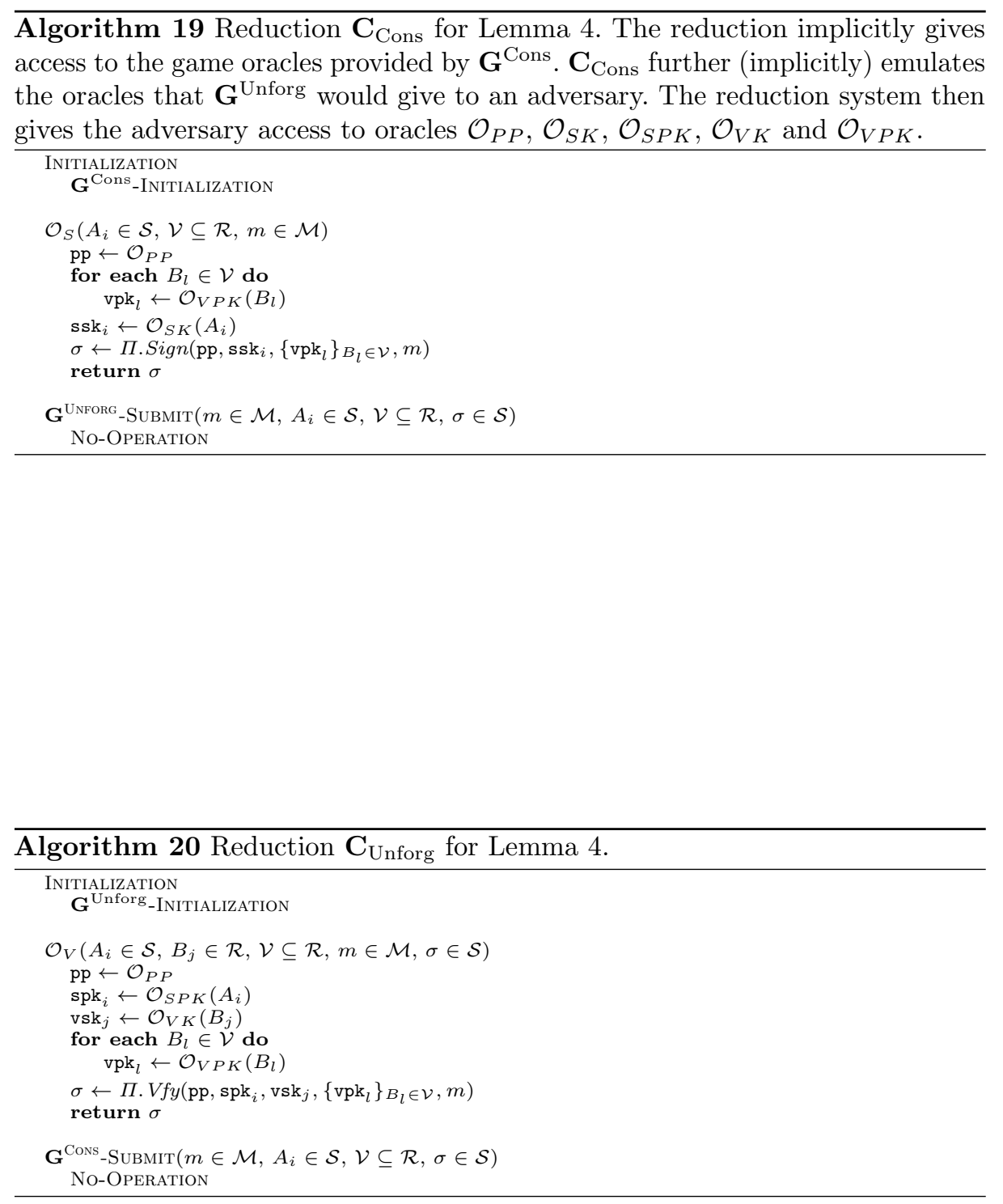
$\mathbf{S}^{\prime}$ denote the ideal repository

$$
\mathbf{S}^{\prime}:=\left[\begin{array}{c}
{\left[\left\langle A_{i} \rightarrow \mathcal{V}\right\rangle_{\mathcal{V} \cup \overline{\mathcal{P}^{H}}}^{\overline{\mathcal{P}^{H}}}\right]_{A_{i} \in \overline{\mathcal{S}^{H}}, \mathcal{V} \subseteq \mathcal{R}}} \\
{\left[\overline{\mathcal{P}^{H}}\left\langle A_{i} \rightarrow \mathcal{V}\right\rangle_{\mathcal{V} \cup \overline{\mathcal{P}^{H}}}^{\left\{A_{i}\right.}\right]_{A_{i} \in \mathcal{S}^{H}, \mathcal{V} \subseteq \mathcal{R}}}
\end{array}\right],
$$

and $\mathbf{S}$ denote the ideal world system

$$
\mathbf{S}:=\operatorname{sim}^{\overline{\mathcal{P}^{H}}} \mathbf{S}^{\prime} .
$$

Consider the following events:

$\xi_{1} \mathbf{D}$ performs a WRITE operation with input $\left(m^{*}, \sigma^{*},\left(A_{i}{ }^{*}, \mathcal{V}^{*}\right)\right)$, where $A_{i}{ }^{*} \in$ $\overline{\mathcal{S}^{H}}$, at one of the adversarial interfaces $\overline{\mathcal{P}^{H}}$, returning an identifier id, such that there are two later READBUfFER invocations at interfaces $B_{h}$ and $B_{h^{\prime}}$ (where $B_{h}, B_{h^{\prime}} \in \mathcal{V}^{H}$ ) such that one of them returns a list containing an entry of the form $(\mathrm{id}, \cdot)$ and the other returns a list not containing any such entry;

$\xi_{2} \mathbf{D}$ performs a WRITE operation with input $\left(m^{*}, \sigma^{*},\left(A_{i}{ }^{*}, \mathcal{V}^{*}\right)\right)$, where $A_{i}{ }^{*} \in$ $\mathcal{S}^{H}$ at one of the adversarial interfaces $\overline{\mathcal{P}^{H}}$, returning an identifier id, such that 1. D did not previously issue a WRITE operation at the interface of party $A_{i}{ }^{*} \in \mathcal{S}^{H}$ for $\left\langle A_{i}{ }^{*} \rightarrow \mathcal{V}^{*}\right\rangle$ with input $m^{*}$, and 2. there is a later READBUfFER invocation at the interface of some (honest) receiver $B_{j} \in \mathcal{V}^{H^{*}}$ for $\left\langle A_{i}{ }^{*} \rightarrow \mathcal{V}^{*}\right\rangle$ in which a list containing an entry of the form $(\mathrm{id}, \cdot)$ is returned;

$\xi_{3} \mathbf{D}$ performs a WRITE operation at the interface of party $A_{i} \in \mathcal{S}^{H}$ giving as input label $\left\langle A_{i}{ }^{*} \rightarrow \mathcal{V}^{*}\right\rangle$ and some message $m$, and the operation returns an identifier id such that there is a later READBUfFER invocation at the interface of some (honest) receiver $B_{j} \in \mathcal{V}^{H^{*}}$ in which there is no entry of the form $(i d, \cdot)$ in the list returned by the invocation.

It is easy to see, from an argument along the lines of the ones in Lemma 1 and in Lemma 2, that $\mathbf{R} \equiv^{\xi} \mathbf{S}$, for $\xi=\xi_{1} \vee \xi_{2} \vee \xi_{3}$. Furthermore, note that the three events defined above, $\xi_{1}, \xi_{2}$, and $\xi_{3}$, can only occur when $\mathbf{D}$ is interacting with the real world system $\mathbf{R}$, meaning that $\operatorname{Pr}^{\mathbf{D S}}[\xi]=0$. With this, we now bound the probability for $\xi$ to occur.

Consider the reduction systems $\mathbf{C}$ (see Algorithm 18), $\mathbf{C}_{\text {Cons }}$ (see Algorithm 19 and $\mathbf{C}_{\text {Unforg }}$ (see Algorithm 20). First, note that $\mathbf{C}_{\text {Cons }} \mathbf{G}^{\text {Cons }} \equiv$ $\mathbf{C}_{\text {Unforg }} \mathbf{G}^{\text {Unforg. }}$. Furthermore, analogously to Lemma 2 and to Lemma 1, it is easy to see, on one hand, that $\mathbf{R} \equiv \mathbf{C C}_{\text {Cons }} \mathbf{G}^{\mathrm{Cons}} \equiv \mathbf{C C}_{\mathrm{Unforg}} \mathbf{G}^{\text {Untorg, }}$, and on the other hand that

$\xi_{1}$ the probability for $\xi_{1}$ to occur is bounded by the probability that adversary DCC $_{\text {Cons }}$ wins $\mathbf{G}^{\text {Cons }}$ (as all the conditions for winning the consistency game are met), implying

$$
\operatorname{Pr}^{\mathbf{D R}}\left[\xi_{1}\right]=\operatorname{Pr}^{\mathbf{D C C}} \mathbf{C o n s}^{\text {Gons }}\left[\xi_{1}\right] \leq A d v^{\mathrm{Cons}}\left(\mathbf{D C C}_{\mathrm{Cons}}\right) ;
$$


$\xi_{2}$ the probability that $\xi_{2}$ occurs is bounded by advantage of adversary $\mathbf{D C} \mathbf{C}_{\mathrm{Unforg}}$ in winning $\mathbf{G}^{\text {Unforg }}$ (as all the winning conditions for the unforgeability game are met), implying

$$
\operatorname{Pr}^{\mathbf{D R}}\left[\xi_{2}\right]=\operatorname{Pr}^{\mathbf{D C C}} \mathbf{U n f o r g}^{\mathbf{G}^{\text {Unforg }}}\left[\xi_{2}\right] \leq A d v^{\text {Unforg }}\left(\mathbf{D C C}_{\text {Unforg }}\right) ;
$$

$\xi_{3}$ there is an explicit reduction system $\mathbf{C}^{\prime}$ satisfying $\mathbf{R} \equiv \mathbf{C}^{\prime} \mathbf{G}^{\text {Corr }}$ and for which the probability for $\xi_{3}$ to occur is bounded by the probability that adversary $\mathbf{D C}^{\prime}$ wins $\mathbf{G}^{\text {Corr }}$ (as all the conditions for winning the correctness game are met), implying

$$
\operatorname{Pr}^{\mathbf{D R}}\left[\xi_{3}\right]=\operatorname{Pr}^{\mathbf{D C}^{\prime} \mathbf{G}^{\mathrm{Corr}}}\left[\xi_{3}\right] \leq A d v^{\mathrm{Corr}}\left(\mathbf{D C}^{\prime}\right) .
$$

Since, as already mentioned, $\operatorname{Pr}^{\mathbf{D S}}[\xi]=0$, we then have

$$
\begin{aligned}
\left|\Delta^{\mathbf{D}}(\mathbf{R}, \mathbf{S})\right| & \leq \operatorname{Pr}^{\mathbf{D R}}[\xi] \\
& \leq \operatorname{Pr}^{\mathbf{D R}}\left[\xi_{1}\right]+\operatorname{Pr}^{\mathbf{D R}}\left[\xi_{2}\right]+\operatorname{Pr}^{\mathbf{D R}}\left[\xi_{2}\right] \\
& \leq A d v^{\text {Cons }}\left(\mathbf{D C C}_{\text {Cons }}\right)+A d v^{\text {Unforg }}\left(\mathbf{D C C}_{\text {Unforg }}\right)+A d v^{\text {Corr }}\left(\mathbf{D C}^{\prime}\right) .
\end{aligned}
$$

\section{A.5 Proof of Theorem 5}

To prove Theorem 5 we prove the following result.

Lemma 5. Consider a setting where $\mathcal{R}^{H}, \overline{\mathcal{R}^{H}}, \mathcal{S}^{H}$ and $\overline{\mathcal{S}^{H}}$ are all non-empty. For any signature forgery algorithm Forge suitable for the Off-The-Record security notion (see Definition 5), there is a protocol $\pi^{\text {Forge }}$ (see Algorithm 10) such that reduction systems $\mathbf{C}$ (see Algorithm 22) and $\mathbf{C}^{\prime}$ (see Algorithm 23) and simulator sim (see Algorithm 21) satisfy, for any distinguisher $\mathbf{D}$

$$
\begin{aligned}
& \mid \Delta^{\mathbf{D}}\left(\left(\pi^{\text {Forge }}\right)^{\overline{\mathcal{S}^{H}} \cup \overline{\mathcal{R}^{H}}}\left(\perp_{\mathrm{Arb}}\right)^{\mathcal{R}^{H}}\left(\mathrm{Snd}^{\mathrm{Arb}} \mathcal{S}^{H} \operatorname{Rcv}^{\mathrm{Arb} \mathcal{R}^{H}}\{[\text { KGA }, \mathbf{I N S}]\}\right),\right. \\
& \left.\operatorname{sim}^{\{E\}}\left[\begin{array}{c}
{\left[\left\langle A_{i} \rightarrow \mathcal{V}\right\rangle_{\mathcal{V}^{H}}^{\{E\}}\right]_{A_{i} \in \overline{\mathcal{S}^{H}}, \mathcal{V} \subseteq \mathcal{R}}} \\
{\left[\left\langle A_{i} \rightarrow \mathcal{V}\right\rangle_{\{E\}}^{\left\{A_{i}\right\} \cup \mathcal{S}^{H} \cup \overline{\mathcal{R}^{H}}}\right]_{A_{i} \in \mathcal{S}^{H}, \mathcal{V} \subseteq \mathcal{R}}}
\end{array}\right]\right) \mid \\
& \leq A d v^{\text {OTR-Forge }}(\mathbf{D C})+A d v^{\text {Cons }}\left(\mathbf{D C}^{\prime}\right) \text {. }
\end{aligned}
$$

Proof. As specified in Algorithm 10, when a party $P \in \overline{\mathcal{S}^{H}} \cup \overline{\mathcal{R}^{H}}$ invokes method Write to $\pi^{\text {Forge }}$ giving as input a label $\left\langle A_{i} \rightarrow \mathcal{V}\right\rangle$ and a message $m \in \mathcal{M}, \pi^{\text {Forge }}$ first fetches the public parameters, the public keys of the signer $A_{i}$ and of each of the designated verifiers in $\mathcal{V}$, and the secret keys of each of the dishonest verifiers (if any), and then uses these to simulate a signature $\sigma$ on $m$. Finally, the converter writes $\left(m, \sigma,\left(A_{i}, \mathcal{V}\right)\right)$ into the insecure repository. 


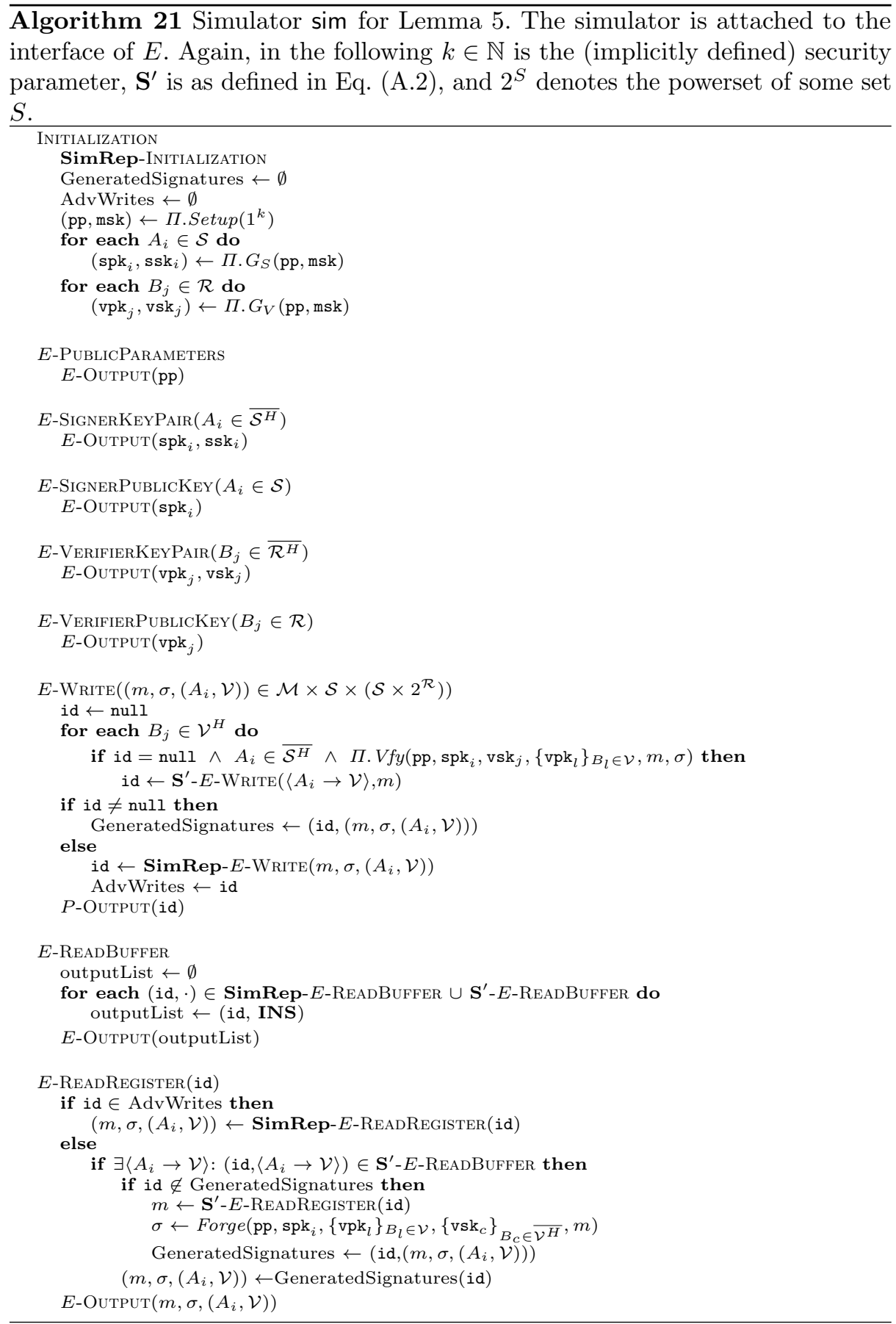




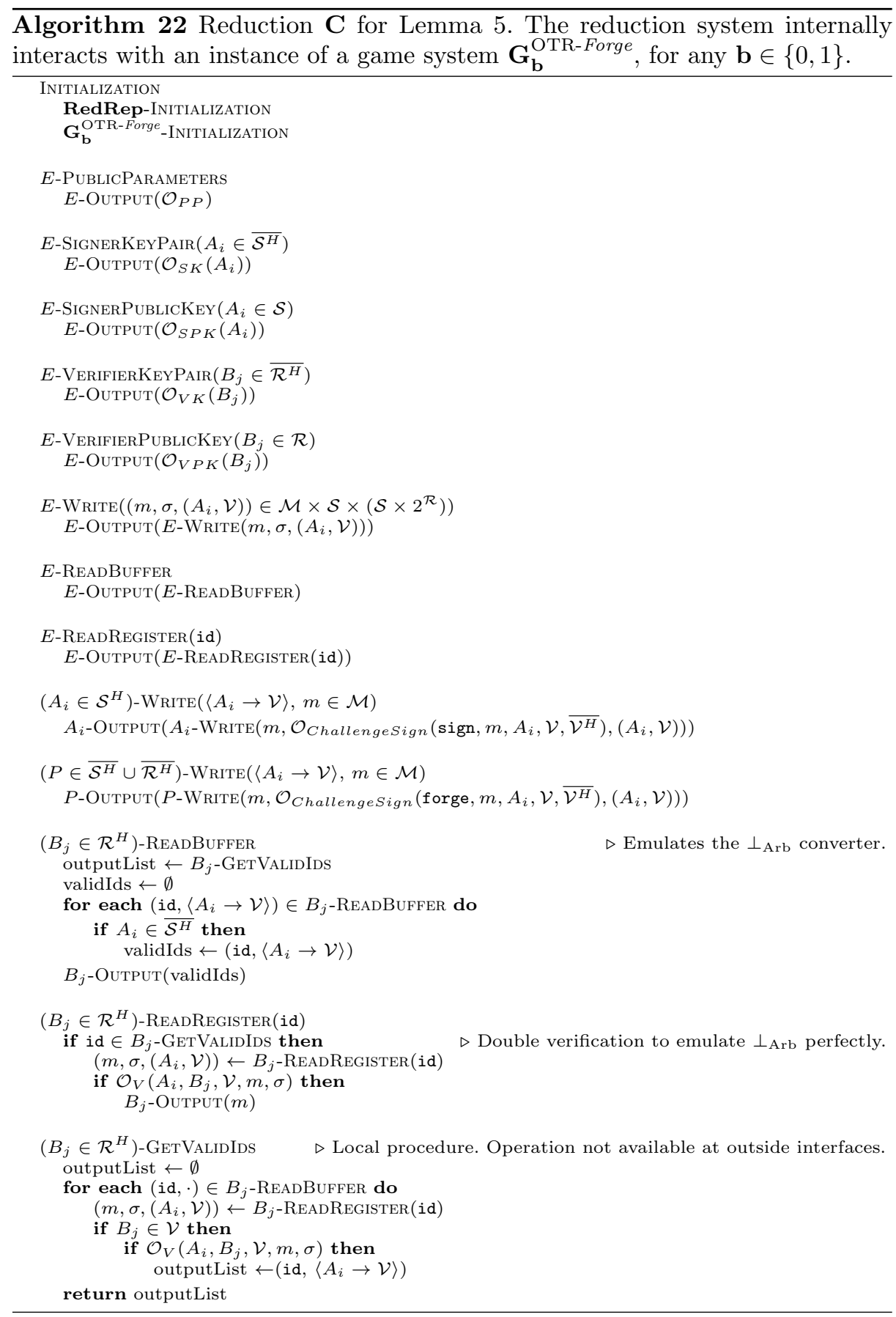




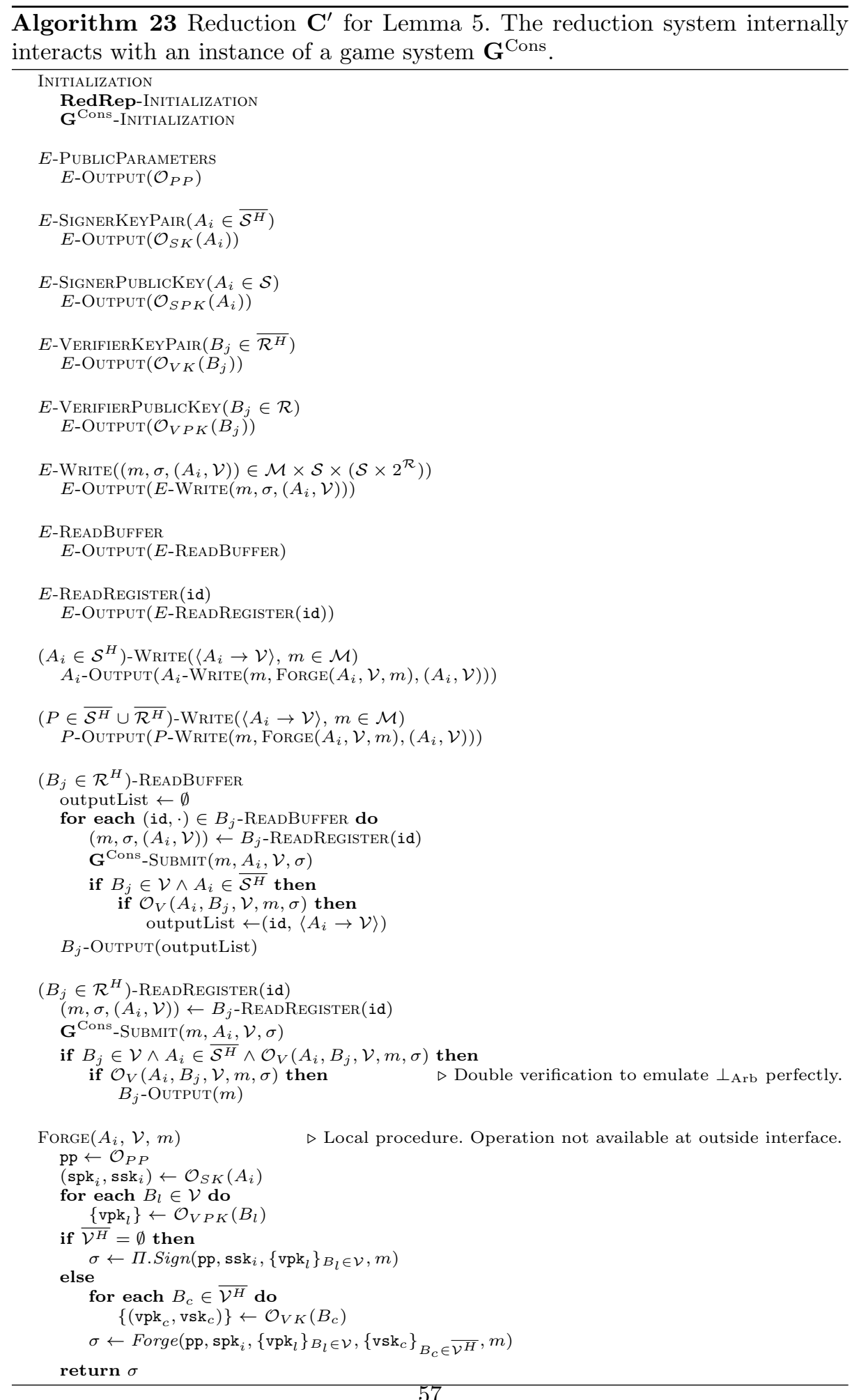


Simulator sim (specified in Algorithm 21) initially generates the necessary keys and public parameters. Additionally, sim contains an internal repository SimRep := SimRep $\{E\}$ to which $E$ has both read and write access. More, the simulator also stores a mapping GeneratedSignatures from register ids to signatures, and a set AdvWrites of ids of registers generated by adversarial writes.

Let $\mathbf{R}$ denote the real world system

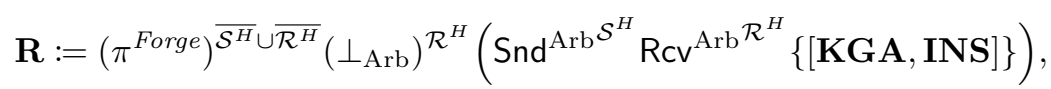

$\mathbf{S}^{\prime}$ denote the ideal repository

$$
\mathbf{S}^{\prime}:=\left[\begin{array}{c}
{\left[\left\langle A_{i} \rightarrow \mathcal{V}\right\rangle_{\mathcal{V}^{H}}^{\{E\}}\right]_{A_{i} \in \overline{\mathcal{S}^{H}}, \mathcal{V} \subseteq \mathcal{R}}} \\
{\left[\left\langle A_{i} \rightarrow \mathcal{V}\right\rangle_{\{E\}}^{\left\{A_{i}\right\} \cup \overline{\mathcal{S}}^{H} \cup \overline{\mathcal{R}^{H}}}\right]_{A_{i} \in \mathcal{S}^{H}, \mathcal{V} \subseteq \mathcal{R}}}
\end{array}\right],
$$

and $\mathbf{S}$ denote the ideal world system

$$
\mathbf{S}:=\operatorname{sim}^{\{E\}} \mathbf{S}^{\prime} .
$$

Let $\xi$ denote the event that $\mathbf{D}$ issues a WRITE operation at the interface of $E$ with input $\left(m^{*}, \sigma^{*},\left(A_{i}{ }^{*}, \mathcal{V}^{*}\right)\right)$, where $A_{i}{ }^{*} \in \overline{\mathcal{S}^{H}}$, returning an identifier id, such that there are two later READBUFFER invocations at interfaces $B_{h}$ and $B_{h^{\prime}}$ (where $B_{h}, B_{h^{\prime}} \in \mathcal{V}^{H}$ ) with one of them returning a list containing an entry of the form $(i d, \cdot)$ and the other returning a list not containing any such entry. It is easy to see that $\operatorname{Pr}^{\mathbf{D S}}[\xi]=0$.

Reduction system $\mathbf{C}$, specified in Algorithm 22, has an internal repository RedRep that it uses to store message signature pairs. $\mathbf{C}$ relies on the oracles provided by the game systems to emulate the KGA resource and uses the $\mathcal{O}_{\text {ChallengeSign }}$ oracle to get signatures on messages that are inserted at the interfaces of honest senders in $\mathcal{S}^{H}$ and of dishonest senders and receivers in $\overline{\mathcal{S}^{H}} \cup \overline{\mathcal{R}^{H}}$. As in Lemma 3, it is easy to see that $\mathbf{C}$ perfectly emulates:

- the real world $\mathbf{R}$ when connected to the game system $\mathbf{G}_{\mathbf{0}}^{\text {OTR-Forge }}$ (i.e. $\mathbf{R} \equiv$ $\mathbf{C G}_{\mathbf{0}}^{\text {OTR-Forge }}$;

- the ideal world $\mathbf{S}$ when connected to $\mathbf{G}_{\mathbf{1}}^{\text {OTR-Forge }}$ as long as event $\xi$ does not occur (i.e. $\mathbf{S} \equiv{ }^{\xi} \mathbf{C G}_{\mathbf{1}}^{\text {OTR-Forge }}$ ).

To conclude the proof, we now bound the probability for $\xi$ to occur. The reduction system $\mathbf{C}^{\prime}$ specified in Algorithm 23 has an internal repository RedRep, and emulates $\mathbf{C G}_{\mathbf{1}}^{\text {OTR-Forge }}$ when connected to the consistency game $\mathbf{G}^{\text {Cons }}$. During the emulation, $\mathbf{C}$ tries to win the game by submitting inputs from $\mathbf{D}$ as challenges to the game. It is easy to see from the specification of $\mathbf{C}^{\prime}$ (Algorithm 23) that the behaviors of $\mathbf{C G}_{\mathbf{1}}^{\text {OTR-Forge }}$ and of $\mathbf{C}^{\prime} \mathbf{G}^{\text {Cons }}$ are perfectly indistinguishable, and that the probability that $\xi$ occurs is upper bounded by the probability that the adversary $\mathbf{D} \mathbf{C}^{\prime}$ wins $\mathbf{G}^{\text {Cons }}$ (as all the conditions for winning the game are met). Thus, we have

$$
\operatorname{Pr}^{\mathbf{D C G}_{1}^{\text {OTR-Forge }}}[\xi]=\operatorname{Pr}^{\mathbf{D C}^{\prime} \mathbf{G}^{\text {Cons }}}[\xi] \leq A d v^{\text {Cons }}\left(\mathbf{D C}^{\prime}\right),
$$


implying, since $\operatorname{Pr}^{\mathbf{D S}}[\xi]=0$, that

$$
\left|\Delta^{\mathbf{D}}\left(\mathbf{C G}_{\mathbf{1}}^{\text {OTR-Forge }}, \mathbf{S}\right)\right|=\left|\Delta^{\mathbf{D}}\left(\mathbf{C}^{\prime} \mathbf{G}^{\text {Cons }}, \mathbf{S}\right)\right| \leq A d v^{\text {Cons }}\left(\mathbf{D C}^{\prime}\right) .
$$

Finally, for any distinguisher $\mathbf{D}$,

$$
\begin{aligned}
\left|\Delta^{\mathbf{D}}(\mathbf{R}, \mathbf{S})\right| \leq & \left|\Delta^{\mathbf{D}}\left(\mathbf{R}, \mathbf{C G}_{\mathbf{0}}^{\text {OTR-Forge }}\right)\right| \\
+ & \left|\Delta^{\mathbf{D}}\left(\mathbf{C G}_{\mathbf{0}}^{\text {OTR-Forge }}, \mathbf{C G}_{\mathbf{1}}^{\text {OTR-Forge }}\right)\right| \\
+ & \left|\Delta^{\mathbf{D}}\left(\mathbf{C G}_{\mathbf{1}}^{\text {OTR-Forge }}, \mathbf{S}\right)\right| \\
= & \mid \operatorname{Pr}\left[\mathbf{D C} \mathbf{D G}_{\mathbf{1}}^{\text {OTR-Forge }}=\text { win }\right] \\
& +\operatorname{Pr}\left[\mathbf{D C G} \mathbf{C G}_{\mathbf{0}}^{\text {OTR-Forge }}=\text { win }\right]-1 \mid \\
& +A d v^{\text {Cons }}\left(\mathbf{D C}^{\prime}\right) \\
= & A d v^{\text {OTR-Forge }}(\mathbf{D C})+A d v^{\text {Cons }}\left(\mathbf{D C}^{\prime}\right) .
\end{aligned}
$$

\section{A.6 Proof of Theorem 6}

Proof. In the following, we (implicitly) assume that the set of parties $\mathcal{P}$ is such that all of $\mathcal{R}^{H}, \overline{\mathcal{R}^{H}}, \mathcal{S}^{H}$ and $\overline{\mathcal{S}^{H}}$ are non-empty sets. We also assume $A_{1} \in \mathcal{S}^{H}$, $B_{1} \in \overline{\mathcal{R}^{H}}$, and $B_{2} \in \mathcal{R}^{H}$.

We construct $\Pi^{\prime}$ from $\Pi$ as follows: $\Pi^{\prime}=\left(S, G_{S}, G_{V}\right.$, Sign $\left.^{\prime}, V_{f y}^{\prime}\right)$, where Sign $^{\prime}$ and $V f y^{\prime}$ internally use Sign and Vfy, respectively, but where Sign' now appends an extra bit 0 to each signature generated by Sign and $V f y^{\prime}$ ignores this last bit and internally uses Vfy to verify the signature.

It is easy to see via a reduction argument that if $\Pi$ is secure as in Theorem 4

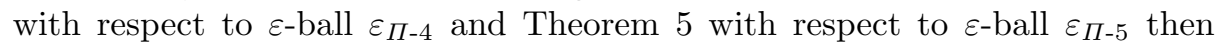
$\Pi^{\prime}$ is also secure as in each of these two theorems and for the same $\varepsilon$-balls as $\Pi$, i.e. $\varepsilon_{\Pi \square}$ for Theorem 4 and $\varepsilon_{\Pi \square}$ for Theorem 5 -note that any signature forgery algorithm Forge assumed to exist for $\Pi$ can be trivially adapted into one suitable for $\Pi^{\prime}$, say Forge ${ }^{\prime}$ then yielding an explicit protocol $\pi^{\text {Forge }}$ as required by Theorem 5 .

We now show that an adversary $\mathbf{A}^{m}$ playing the Off-The-Record game for MDVS scheme $\Pi^{\prime}$ will win the game with high probability. $\mathbf{A}^{m}$ behaves as follows. It submits a query

$$
\left.\mathcal{O}_{\text {ChallengeSign }} \text { (sign, } m, A_{1},\left\{B_{1}, B_{2}\right\},\left\{B_{1}\right\}\right),
$$

where $A_{1}$ is an honest signer, $B_{1}$ a dishonest verifier and $B_{2}$ an honest verifier. Then $\mathbf{A}^{m}$ gets $\sigma \| 0$ as output from the game. Next, $\mathbf{A}^{m}$ submits a query

$$
\mathcal{O}_{V}\left(A_{1}, B_{2},\left\{B_{1}, B_{2}\right\}, m, \sigma \| 1\right) .
$$

If $\sigma \| 1$ is a valid signature for $m$, then $\mathcal{O}_{V}$ outputs 1 and $\mathbf{A}^{m}$ guesses that it is interacting with $\mathbf{G}_{\mathbf{0}}^{\text {OTR-Forge }}$ since this game produces a valid signature. But if 
the signature was generated by the Forge algorithm, i.e. $\mathbf{A}^{m}$ is interacting with $\mathbf{G}_{\mathbf{1}}^{\text {OTR-Forge }}$, then the signature will be recognized as invalid by $\mathcal{O}_{V}$ and $\mathbf{A}^{m}$ will make the corresponding guess.

The proof is however not completely straightforward, because the scheme might not have perfect correctness or perfect authenticity: there might be a chance that $\mathbf{G}_{\mathbf{0}}^{\text {OTR-Forge }}$ produces an invalid signature (when correctness fails) or that $\mathbf{G}_{\mathbf{1}}^{\text {OTR-Forge }}$ produces a valid signature (when authenticity fails).

Let $\delta_{\text {corr }}$ denote the probability that the signature produced is invalid (for honest receiver $\left.B_{2}\right)$, i.e. for $\sigma=\operatorname{Sign}\left(\mathrm{pp}, \mathrm{ssk}_{1},\left\{\mathrm{vpk}_{1}, \mathrm{vpk}_{2}\right\}, m\right)$,

$$
\delta_{\text {corr }}=\operatorname{Pr}\left[V f y\left(\mathrm{pp}, \mathrm{spk}_{1}, \mathrm{vsk}_{2},\left\{\mathrm{vpk}_{1}, \mathrm{vpk}_{2}\right\}, m, \sigma\right)=0\right] \text {. }
$$

And let $\delta_{\text {auth }}$ denote the probability that the signature produced by the forgery algorithm is valid (to honest receiver $B_{2}$ ), i.e. for

$$
\begin{gathered}
\sigma=\operatorname{Forge}\left(\mathrm{pp}, \mathrm{spk}_{1},\left\{\mathrm{vpk}_{1}, \mathrm{vpk}_{2}\right\},\left\{\mathrm{vsk}_{1}\right\}, m\right), \\
\delta_{\text {auth }}=\operatorname{Pr}\left[V f y\left(\mathrm{pp}, \mathrm{spk}_{1}, \mathrm{vsk}_{2},\left\{\mathrm{vpk}_{1}, \mathrm{vpk}_{2}\right\}, m, \sigma\right)=1\right] .
\end{gathered}
$$

We then have

$$
\operatorname{Pr}\left[\mathbf{A}^{m} \mathbf{G}_{\mathbf{0}}^{\text {OTR-Forge }}=\text { lose }\right]=\delta_{\text {corr }}
$$

and

$$
\operatorname{Pr}\left[\mathbf{A}^{m} \mathbf{G}_{\mathbf{1}}^{\text {OTR-Forge }}=\text { lose }\right]=\delta_{\text {auth }}
$$

Hence,

$$
\begin{aligned}
& A d v^{\Pi^{\prime} \text {-OTR-Forge }}\left(\mathbf{A}^{m}\right)=\mid \operatorname{Pr}\left[\mathbf{A}^{m} \mathbf{G}_{\mathbf{0}}^{\text {OTR-Forge }}=\text { win }\right] \\
& +\operatorname{Pr}\left[\mathbf{A}^{m} \mathbf{G}_{\mathbf{1}}^{\text {OTR-Forge }}=\text { win }\right]-1 \\
& =\mid 1-\operatorname{Pr}\left[\mathbf{A}^{m} \mathbf{G}_{\mathbf{0}}^{\text {OTR-Forge }}=\text { lose }\right] \\
& +1-\operatorname{Pr}\left[\mathbf{A}^{m} \mathbf{G}_{\mathbf{1}}^{\text {OTR-Forge }}=\text { lose }\right] \\
& -1 \\
& =1-\operatorname{Pr}\left[\mathbf{A}^{m} \mathbf{G}_{\mathbf{0}}^{\text {OTR-Forge }}=\text { lose }\right] \\
& -\operatorname{Pr}\left[\mathbf{A}^{m} \mathbf{G}_{\mathbf{1}}^{\text {OTR-Forge }}=\text { lose }\right] \\
& =1-\delta_{\text {corr }}-\delta_{\text {auth }} \text {. }
\end{aligned}
$$

